\title{
Energy Bounds and Vanishing Results for the Gromov-Witten Invariants of the Projective Space
}

\author{
Aleksey Zinger* \\ January 23, 2018
}

\begin{abstract}
We describe generating functions for arbitrary-genus Gromov-Witten invariants of the projective space with any number of marked points explicitly. The structural portion of this description gives rise to uniform energy bounds and vanishing results for these invariants. They suggest deep conjectures relating Gromov-Witten invariants of symplectic manifolds to the energy of pseudo-holomorphic maps and the expected dimension of their moduli space.
\end{abstract}

\section{Contents}

1 Introduction $\quad 2$

2 Main theorem and some applications 5

2.1 Structure of Hodge integrals . . . . . . . . . . . . . . . . . . 6

2.2 Asymptotic expansions . . . . . . . . . . . . . . . . . 8

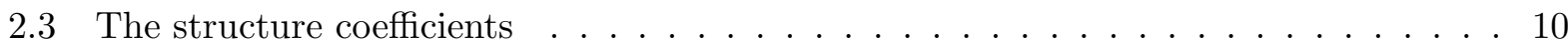

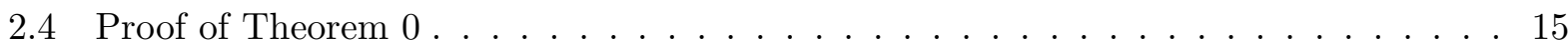

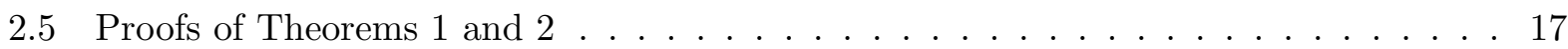

3 Torus equivariant setting $\quad 23$

3.1 Equivariant GW-invariants . . . . . . . . . . . . . . . . . 23

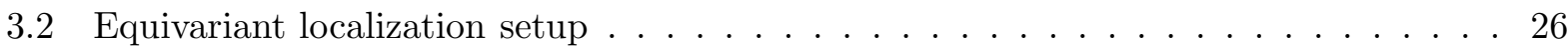

3.3 Outline of proofs of Theorem $\mathrm{B} \ldots \ldots \ldots \ldots \ldots \ldots \ldots$

3.4 Key equivariant inputs . . . . . . . . . . . . . . . . . . . 31

4 Proof of Theorem B 35

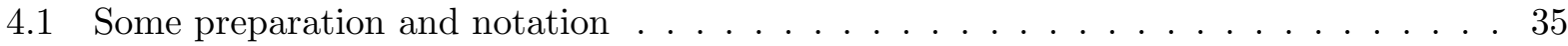

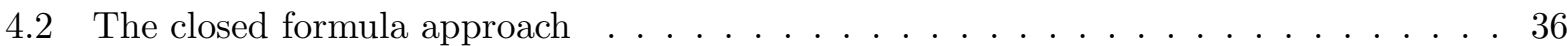

4.3 The recursion approach $\ldots \ldots \ldots \ldots \ldots \ldots$

5 Key combinatorial identities $\quad 48$

5.1 Proof of Proposition $2.1 \ldots \ldots \ldots \ldots \ldots \ldots \ldots \ldots$

5.2 Sums of residues of generating series . . . . . . . . . . . . . 54

*Partially supported by NSF grant 1500875 and MPIM 


\section{Introduction}

Gromov-Witten (or GW-) invariants of a smooth projective variety (or more generally of a symplectic manifold) $X$ are certain counts of (pseudo-holomorphic) curves in $X$. These invariants are known or conjectured to possess many striking properties which are often completely unexpected from the classical point of view. For example, physical considerations suggest that these invariants are uniformly bounded by the symplectic area of the curves being counted; see Conjecture 1, We confirm this conjecture for the complex projective space $\mathbb{P}^{n-1}$ in all genera by applying the explicit formula of Theorem $\mathrm{A}$ in Section 2.3. We also use this theorem to confirm the vanishing predictions of Conjecture 2 for $\mathbb{P}^{n-1}$.

Generating functions for the 1-pointed genus $0 \mathrm{GW}$-invariants of semi-positive projective complete intersections $X \subset \mathbb{P}^{n-1}$ are explicitly computed in [8, 13. The resulting formulas in particular confirm the mirror symmetry prediction of [4] for the genus $0 \mathrm{GW}$-invariants of a quintic threefold, i.e. a degree 5 hypersurface in $\mathbb{P}^{4}$. By [3, 21], generating functions for 2-pointed genus $0 \mathrm{GW}$ invariants of hypersurfaces are explicit transforms of the 1-pointed genus 0 functions; these results are extended to projective complete intersections in [5, 18, and to complete intersections in toric varieties in [17]. It is shown in [22] that generating functions for $N$-pointed genus $0 \mathrm{GW}$-invariants of projective complete intersections, with $N \geq 3$, are also explicit transforms of the 1-pointed genus 0 functions. Combined with [20, 16], this implies the same for generating functions for $N$-pointed genus $1 \mathrm{GW}$-invariants of projective complete intersections. We show in this paper that a natural generating function for the $N$-pointed genus $g \mathrm{GW}$-invariants of $\mathbb{P}^{n-1}$ is an explicit transform of the 1-pointed genus 0 generating function as well; see Theorem $\mathrm{A}$ in Section 2.3 and Theorem B in Section 3 ,

Throughout the paper $n, g, N \in \mathbb{Z}^{+}$are fixed integers, with $g$ and $N$ denoting the genus of the curves being counted and the number of marked points, respectively. Let

$$
\mathbb{Z}^{\geq 0}=\{0\} \sqcup \mathbb{Z}^{+} \quad \text { and } \quad[N]=\{1,2, \ldots, N\} .
$$

For $d \in \mathbb{Z}^{\geq 0}$, we denote by $\overline{\mathfrak{M}}_{g, N}\left(\mathbb{P}^{n-1}, d\right)$ the moduli space of stable $N$-marked genus $g$ degree $d$ maps to $\mathbb{P}^{n-1}$. For each $s=1, \ldots, N$, let

$$
\mathrm{ev}_{s}: \overline{\mathfrak{M}}_{g, N}\left(\mathbb{P}^{n-1}, d\right) \longrightarrow \mathbb{P}^{n-1}, \quad \psi_{s} \equiv c_{1}\left(L_{s}^{*}\right) \in H^{2}\left(\overline{\mathfrak{M}}_{g, N}\left(\mathbb{P}^{n-1}, d\right)\right),
$$

be the evaluation map and the first Chern of the universal cotangent line bundle at the $s$-th marked point, respectively. Denote by $H \in H^{2}\left(\mathbb{P}^{n-1}\right)$ the hyperplane class.

The main theorem of this paper, Theorem A stated at the beginning of Section 2, provides a closed formula for the $N$-pointed genus $g$ version of the standard (1-pointed genus 0) Givental's $J$-function. This is a generating function for the genus $g \mathrm{GW}$-invariants

$$
\left\langle\tau_{b_{1}} H^{c_{1}}, \ldots, \tau_{b_{N}} H^{c_{N}}\right\rangle_{g, d}^{\mathbb{P}^{n-1}} \equiv \int_{\left.\left[\overline{\mathfrak{M}}_{g, N}\left(\mathbb{P}^{n-1}, d\right)\right]\right]^{\mathrm{vir}}} \prod_{s=1}^{s=N}\left(\psi_{s}^{b_{s}} \operatorname{ev}_{s}^{*} H^{c_{s}}\right)
$$

of $\mathbb{P}^{n-1}$. The most basic positive-genus case of Theorem $\mathrm{A}$ is equivalent to Theorem 0 below. 
Theorem 0. For all $n, d, c \in \mathbb{Z}^{\geq 0}$ with $c<n$ and $n \geq 2$,

$$
\left\langle\tau_{n d+1-c} H^{c}\right\rangle_{1, d}^{\mathbb{P}^{n-1}}=\llbracket \frac{n(1+2 d-n+2 w)}{48} \frac{(d+w)^{n-2}}{\prod_{r=1}^{d}(r+w)^{n}} \rrbracket_{w ; n-1-c},
$$

with $\llbracket f \rrbracket_{w ; r}$ denoting the coefficient of $w^{r}$ in the power series expansion of a function $f=f(w)$ around $w=0$.

This theorem is obtained in Section 2.4. While the precise statement of Theorem $\mathrm{A}$ is quite involved in general, its qualitative corollaries, Theorems 1 and 2 below, are quite simple to state; they are established in Section 2.5.

Theorem 1. For all $n \in \mathbb{Z}^{+}$and $g \in \mathbb{Z}^{\geq 0}$, there exists $C_{n, g} \in \mathbb{R}^{+}$such that

$$
\left|\frac{\left\langle b_{1} ! \tau_{b_{1}} H^{c_{1}}, \ldots, b_{N} ! \tau_{b_{N}} H^{c_{N}}\right\rangle_{g, d}^{\mathbb{P}^{n-1}}}{N !}\right| \leq C_{n, g}^{d+N}
$$

for all $N \in \mathbb{Z}^{+}$and $d, b_{1}, \ldots, b_{N}, c_{1}, \ldots, c_{N} \in \mathbb{Z}^{\geq 0}$.

In the basic $d=0$ case, the invariants of Theorem 1 become

$$
\left\langle\tau_{b_{1}} H^{c_{1}}, \ldots, \tau_{b_{N}} H^{c_{N}}\right\rangle_{g, 0}^{\mathbb{P}^{n-1}}=\int_{\overline{\mathcal{M}}_{g, N} \times \mathbb{P}^{n-1}} e\left(\mathbb{E}_{g}^{*} \otimes T \mathbb{P}^{n-1}\right) \prod_{i=1}^{N}\left(\psi_{i}^{b_{i}} H^{c_{i}}\right),
$$

where

$$
\mathbb{E}_{g} \longrightarrow \overline{\mathcal{M}}_{g, N}
$$

is the Hodge vector bundle of holomorphic differentials over the Deligne-Mumford moduli space of genus $g$ curves with $N$ marked points. By (1.2), (2.18), and induction via (5.13),

$$
\left|\frac{\left\langle b_{1} ! \tau_{b_{1}} H^{c_{1}}, \ldots, b_{N} ! \tau_{b_{N}} H^{c_{N}}\right\rangle_{g, 0}^{\mathbb{P}^{n-1}}}{N !}\right| \leq C_{n, g} 2^{N}
$$

for some $C_{n, g} \in \mathbb{R}^{+}$determined by the numbers $C_{g ; n ; I}$ in (2.18) and by the top-dimensional intersections of $\lambda$ and $\psi$-classes on the moduli spaces $\overline{\mathcal{M}}_{g, N}$ with $N \leq 6 g-6$. The base 2 above can be replaced by any number arbitrarily close to 1 at the cost of increasing $C_{n, g}$. The $d=0$ case of Theorem 1 is thus straightforward. The case of Theorem 1 with $n=3, b_{i}=0$ for all $i$, and $c_{i}=2$ for all $i$ is consistent with the asymptotic prediction of [7, Footnote 2] for the number $n_{g, d}$ of genus $g$ degree $d$ curves in $\mathbb{P}^{2}$ passing through $3 d-1+g$ general points.

Theorem 2. Suppose $n, g, N \in \mathbb{Z}^{+}$with $2 g+N \geq 3$ and $\left(b_{s}\right)_{s \in[N]},\left(c_{s}\right)_{s \in[N]} \in\left(\mathbb{Z}^{\geq}\right)^{N}$. If there exists $S \subset[N]$ such that

$$
b_{s}+c_{s}<n \quad \forall s \in S \quad \text { and } \quad \sum_{s \in S} b_{s}>3(g-1)+N,
$$

then $\left\langle\tau_{b_{1}} H^{c_{1}}, \ldots, \tau_{b_{N}} H^{c_{N}}\right\rangle_{g, d}^{\mathbb{P}^{n-1}}=0$ 
Theorems 1 and 2 are potential indications of fundamental properties of GW-invariants that are out of reach of the current methods. Their statements have natural intrinsic extensions to more general symplectic manifolds, formulated in the two conjectures below. The exponent $\langle\omega, \beta\rangle$ in Conjecture 1 is the energy of the $J$-holomorphic maps of class $\beta$, while $\langle\omega, \beta\rangle+N$ is essentially the energy of the induced "graph map". A symplectic manifold $(X, \omega)$ is called monotone with minimal Chern number $\nu \in \mathbb{R}^{+}$in Conjecture 2 if

$$
c_{1}(X)=\lambda[\omega] \in H^{2}(X ; \mathbb{R})
$$

for some $\lambda \in \mathbb{R}^{+}$and $\nu$ is the minimal value of $c_{1}(X)$ on the homology classes representable by non-constant $J$-holomorphic maps $\mathbb{P}^{1} \longrightarrow X$ for every $\omega$-compatible almost complex structure on $X$.

Conjecture 1 ([22, Conjecture 1]). Suppose $(X, \omega)$ is a compact symplectic manifold and $g \in \mathbb{Z}$. For all $H_{1}, \ldots, H_{k} \in H^{*}(X)$, there exists $C_{X, g} \in \mathbb{R}^{+}$such that

$$
\left|\frac{\left\langle b_{1} ! \tau_{b_{1}} H_{c_{1}}, \ldots, b_{N} ! \tau_{b_{N}} H_{c_{N}}\right\rangle_{g, \beta}^{X}}{N !}\right| \leq C_{X, g}^{\langle\omega, \beta\rangle+N} \quad \forall \beta \in H_{2}(X), N, b_{s} \geq 0, c_{s} \in[k] .
$$

Conjecture 2. Suppose $(X, \omega)$ is a compact monotone symplectic manifold with minimal Chern number $\nu$,

$$
g, N \in \mathbb{Z}^{\geq 0} \quad \text { with } 2 g+N \geq 3, \quad\left(b_{s}\right)_{s \in[N]},\left(c_{s}\right)_{s \in[N]} \in\left(\mathbb{Z}^{\geq 0}\right)^{N}, \quad \text { and } H_{s} \in H^{2 c_{s}}(X) \quad \forall s \in[N] .
$$

If there exists $S \subset[N]$ such that

$$
b_{s}+c_{s}<\nu \quad \forall s \in S \quad \text { and } \quad \sum_{s \in S} b_{s}>3(g-1)+N,
$$

then $\left\langle\tau_{b_{1}} H_{1}, \ldots, \tau_{b_{N}} H_{N}\right\rangle_{g, \beta}^{X}=0$.

Theorems 1 and 2 establish Conjectures 1 and 2 for $X=\mathbb{P}^{n-1}$. Theorems 1 and 2 in [22] establish the $g=0$ cases of these conjectures for complete intersections $X \subset \mathbb{P}^{n}$ with each $H_{i}$ being a power of $H$. Conjecture 1 for a Calabi-Yau threefold $X$ corresponds to the string theory presumption that the partition functions determined by GW-invariants have positive radii of convergence. If $X$ is a Calabi-Yau intersection 3 -fold in $\mathbb{P}^{n}$, this is equivalent to the existence of $C_{X, g} \in \mathbb{R}^{+}$such that

$$
\left|\langle\rangle_{g, d}^{X}\right| \leq C_{X, g}^{d} \quad \forall d \in \mathbb{Z}^{+}
$$

For a Calabi-Yau $X$ of (complex) dimension at least 4, the GW-invariants of genus 2 and higher vanish. Conjecture 1 then reduces to its cases for the genus $0 \mathrm{GW}$-invariants with primary insertions $\left(b_{i}=0\right.$ for all $\left.i\right)$ and for the genus $1 \mathrm{GW}$-invariants with no insertions. For complete intersections $X \subset \mathbb{P}^{n}$, such genus 0 bounds with each $H_{i}$ being a power of $H$ are implied by the mirror formulas established in [8, 13]; these mirror formulas and bounds extend to many other GIT quotients. The required genus 1 bounds for complete intersections $X \subset \mathbb{P}^{n}$ are implied by the genus 1 mirror formulas established in [20, 16].

The virtual localization theorem of [10] reduces the computation of positive-genus GW-invariants of $\mathbb{P}^{n-1}$ to a sum over weighted graphs. We use the approach of [20] for breaking such graphs at special nodes to show that a generating function for the $N$-pointed genus $g$ GW-invariants of $\mathbb{P}^{n}$ 
is a linear combination of $N$-fold products of derivatives of a generating function for the 1-pointed genus $0 \mathrm{GW}$-invariants with coefficients that are polynomials of total degree at most $3(g-1)+N$. In contrast to the application of this approach in [22] to compute $N$-pointed genus $0 \mathrm{GW}$-invariants of complete intersections $X \subset \mathbb{P}^{n}$ with $N \geq 3$, the present application requires dealing with graphs containing loops and understanding the structure of Hodge integrals over $\overline{\mathcal{M}}_{g, N}$ as $N$ increases. While we describe two explicit ways of computing the relevant coefficients, the final formulas become rather complicated as $g$ and $N$ increase. Nevertheless, our qualitative description of these coefficients suffices to deduce Theorem 1 and to immediately obtain Theorem 2.

The approach in this paper can be used to compute twisted $N$-pointed genus $g$ GW-invariants of $\mathbb{P}^{n}$, but these do not correspond to the usual GW-invariants of the associated complete intersection for $g \geq 1$. There are two necessary inputs for doing so. The first is Proposition 2.1, which concerns the structure of Hodge integrals only and is thus directly applicable in all cases. The second input is Proposition 2.2, which provides an asymptotic expansion of the mirror hypergeometric function corresponding to the standard Givental's $J$-function. The approach of [19] can be used directly to determine the power series $\xi$ and $\Phi_{b}$ appearing in such expansions in the cases of twisted invariants; in the cases relevant to the projective complete intersections, they are determined in [16].

In principle, all genus $g \mathrm{GW}$-invariants of $\mathbb{P}^{n-1}$ can be determined via [9, Theorem 1]. However, it is unclear how feasible it is to obtain such qualitative conclusions as our Theorems 11 and 2 from [9]. The $g=0$ case of Theorem 1, i.e. [22, Theorem 1], in fact confirmed a conjecture of R. Pandharipande. This statement was part of the idea of [15] to establish the bounds (1.3) in all genera by reducing them to the $n=4$ bounds of Theorem 1 via a degeneration scheme of [14] and reducing the latter bounds to the $g=0$ case via [9. As far as we are aware, the approach of establishing the bounds of Theorem [1 from the $g=0$ case via [9] has not been completed yet.

The main theorem of this paper, Theorem A, is stated at the beginning of Section 2, Two descriptions of the structure coefficients appearing in this theorem are given in Section 2.3 after the two necessary inputs are introduced in Sections 2.1 and 2.2. We compute these coefficients in the $(g, N)=(1,1)$ case explicitly in Section 2.4 and establish Theorem 0 , Theorems 1 and 2 are proved in Section 2.5. The former is deduced directly from the structural description of Theorem A its proof makes no use of Sections 2.1 2.3. Theorem $\mathrm{A}$ is an immediate consequence of its equivariant version, Theorem B, stated in Section 3.1. Section 3.2 applies the virtual localization theorem of [10] to the equivariant $N$-point genus $g$ Givental's $J$-function appearing of Theorem $\mathrm{B}$, reducing it to a sum of rational functions in the equivariant weights over infinitely graphs. Section 3.3 describes two approaches for breaking these graphs at special vertices and reducing the associated infinite sum to a sum of finitely many power series. The necessary equivariant inputs for the resulting finite sums are collected in Section 3.4. After a quick preparation in Section 4.1, Sections 4.2 and 4.3 implement the two approaches outlined in Section 3.3 and establish Theorem $\mathrm{B}$ with the two descriptions of the structure coefficients of Section 2.3 . Sections 5.1 and 5.2 establish the key combinatorial identities involving Hodge integrals that are used in the proof of Theorem B. Propositions 2.1 and 5.8, respectively.

\section{Main theorem and some applications}

For $n, N \in \mathbb{Z}^{\geq 0}$, let

$$
\llbracket n \rrbracket=\left\{p \in \mathbb{Z}^{\geq 0}: p<n\right\}, \quad \mathbb{P}_{N}^{n-1}=\left(\mathbb{P}^{n-1}\right)^{N} .
$$


For each $s=1, \ldots, N$, we set

$$
H_{s}=\pi_{s}^{*} H \in H^{2}\left(\mathbb{P}_{N}^{n-1}\right),
$$

where $\pi_{s}: \mathbb{P}_{N}^{n-1} \longrightarrow \mathbb{P}^{n-1}$ is the projection onto the $s$-th coordinate. If in addition $g, d \in \mathbb{Z}^{\geq 0}$, the virtual fundamental class of $\overline{\mathfrak{M}}_{g, N}\left(\mathbb{P}^{n-1}, d\right)$ determines a cohomology push-forward

$$
\mathrm{ev}_{*}^{d} \equiv\left\{\mathrm{ev}_{1} \times \ldots \times \mathrm{ev}_{N}\right\}_{*}: H^{*}\left(\overline{\mathfrak{M}}_{g, N}\left(\mathbb{P}^{n-1}, d\right)\right) \longrightarrow H^{*}\left(\mathbb{P}_{N}^{n-1}\right) .
$$

With $\underline{\hbar}=\left(\hbar_{1}, \ldots, \hbar_{N}\right), \underline{\hbar}^{-1}=\left(\hbar_{1}^{-1}, \ldots, \hbar_{N}^{-1}\right)$, and $\underline{H}=\left(H_{1}, \ldots, H_{N}\right)$, let

$$
Z^{(g)}(\underline{\hbar}, \underline{H}, q)=\sum_{d=0}^{\infty} q^{d} \operatorname{ev}_{*}^{d}\left\{\prod_{s=1}^{s=N} \frac{1}{\hbar_{s}-\psi_{s}}\right\} \in H^{*}\left(\mathbb{P}_{N}^{n-1}\right)\left[\underline{\hbar}^{-1}\right][[q]] .
$$

This power encodes all descendant $N$-pointed genus $g$ GWs of $\mathbb{P}^{n-1}$ defined in (1.1).

For $\mathbf{b} \equiv\left(b_{s}\right)_{s \in[N]} \in\left(\mathbb{Z}^{\geq 0}\right)^{N}$, a tuple $\hbar$ as above, and $p \in \mathbb{Z}^{\geq 0}$, let

$$
|\mathbf{b}|=\sum_{s=1}^{s=N} b_{s}, \quad \underline{\hbar}^{-\mathbf{b}}=\prod_{s=1}^{s=N}\left(\hbar_{s}^{-1}\right)^{b_{s}}, \quad F_{p}(w, q)=\sum_{d=0}^{\infty} q^{d} \frac{(w+d)^{p} w^{n d-p}}{\prod_{r=1}^{d}(w+r)^{n}} \in \mathbb{Q}(w)[[q]] .
$$

For $\mathbf{p} \equiv\left(p_{s}\right)_{s \in[N]} \in \llbracket n \rrbracket^{N}$ and a tuple $\underline{H}=\left(H_{s}\right)_{s \in[N]}$ of formal variables, define

$$
w_{s}=\frac{H_{s}}{\hbar_{s}}, \quad q_{s}=\frac{q}{H_{s}^{n}}, \quad \Delta_{\mathbf{p}}(\underline{\hbar}, \underline{H}, q)=\prod_{s=1}^{s=N} \frac{H_{s}^{p_{s}}}{\hbar_{s}} F_{p_{s}}\left(w_{s}, q_{s}\right) \in \mathbb{Q}[\underline{H}]\left[\left[\underline{\hbar}^{-1}\right]\right][[q]] .
$$

Theorem A. Suppose $n, N \in \mathbb{Z}^{+}$and $g \in \mathbb{Z}^{\geq 0}$ with $n \geq 2$ and $2 g+N \geq 3$. The generating function (2.1) for the $N$-pointed genus $g G W$-invariants of $\mathbb{P}^{n-1}$ is given by

$$
Z^{(g)}(\underline{\hbar}, \underline{H}, q)=\sum_{\mathbf{p} \in \llbracket n \rrbracket^{N}} \sum_{\substack{\mathbf{b} \in\left(\mathbb{Z}^{\geq 0}\right)^{N} \\|\mathbf{b}| \leq 3(g-1)+N}} \sum_{d=0}^{\infty} \mathrm{c}_{g ; \mathbf{p}, \mathbf{b}}^{(d)} q^{d} \underline{\hbar}^{-\mathbf{b}} \Delta_{\mathbf{p}}(\underline{\hbar}, \underline{H}, q),
$$

with the coefficients $\mathrm{c}_{g ; \mathbf{p}, \mathbf{b}}^{(d)} \in \mathbb{Q}$ as specified in Section 2.3 .

\subsection{Structure of Hodge integrals}

One of the two inputs determining the structure coefficients $\mathbf{c}_{g ; \mathbf{p}, \mathbf{b}}^{(d)}$ in Theorem $\mathrm{A}$ are properties of the Hodge integrals on the Deligne-Mumford moduli spaces $\overline{\mathcal{M}}_{g, N}$ arising from the string and dilaton equations. This input is provided by Proposition 2.1 below; it is proved in Section 5.1 .

Let $g \in \mathbb{Z}^{\geq 0}$. For a tuple $I \equiv\left(i_{1}, i_{2}, \ldots\right)$ in $\mathbb{Z}^{\infty}$ or in $\mathbb{Z}^{g} \subset \mathbb{Z}^{\infty}$, define

$$
|I|=\sum_{k=1}^{\infty} i_{k} \in \mathbb{Z}, \quad\|I\|=\sum_{k=1}^{\infty} k i_{k} \in \mathbb{Z}, \quad \mu_{g}(I)=3(g-1)-\|I\| \in \mathbb{Z} .
$$

If in addition $m \in \mathbb{Z}^{\geq 0}$ with $2 g+m \geq 3$ and $I \in\left(\mathbb{Z}^{\geq 0}\right)^{g}$, let

$$
\lambda_{g ; I}=\prod_{k=1}^{g} c_{k}\left(\mathbb{E}_{g}\right)^{i_{k}} \in H^{2\|I\|}\left(\overline{\mathcal{M}}_{g, m}\right) .
$$


If $m^{\prime} \in \mathbb{Z}^{\geq 0}$ and $\mathbf{b}^{\prime} \in\left(\mathbb{Z}^{\geq 0}\right)^{m^{\prime}}$, denote by $\mathbf{b b}^{\prime}$ the $\left(m+m^{\prime}\right)$-tuple obtained by adjoining $\mathbf{b}^{\prime}$ to $\mathbf{b}$ at the end.

If $\mathbf{b} \equiv\left(b_{k}\right)_{k \in[m]} \in\left(\mathbb{Z}^{\geq 0}\right)^{m}$, let

$$
\begin{gathered}
\left\langle\left\langle\lambda_{g ; I} ; \tau_{\mathbf{b}}\right\rangle\right\rangle= \begin{cases}\int \lambda_{g ; I} \prod_{k=1}^{m} \psi_{k}^{b_{k}}, & \text { if }|\mathbf{b}| \geq \mu_{g}(I)+m ; \\
\overline{\mathcal{M}}_{g,|\mathbf{b}|-\mu_{g}(I)} & \text { if }|\mathbf{b}|<\mu_{g}(I)+m ;\end{cases} \\
\left\langle\left\langle\lambda_{g ; I} ; \widetilde{\tau}_{\mathbf{b}}\right\rangle\right\rangle=\left(\prod_{k=1}^{m} b_{k} !\right)\left\langle\left\langle\lambda_{g ; I} ; \tau_{\mathbf{b}}\right\rangle\right\rangle .
\end{gathered}
$$

For $\mathbf{c} \equiv\left(\mathbf{c}_{r}\right)_{r \in \mathbb{Z}^{+}} \in\left(\mathbb{Z}^{\geq 0}\right)^{\infty}$, let

$$
\begin{gathered}
S(\mathbf{c})=\left\{(r, j) \in \mathbb{Z}^{+} \times \mathbb{Z}^{+}:(r, j) \in\{r\} \times\left[c_{r}\right] \forall r \in \mathbb{Z}^{+}\right\}, \\
A_{I, \mathbf{b} ; \mathbf{c}}^{(g)}=\sum_{\mathbf{b}^{\prime} \in(\mathbb{Z} \geq 0) S(\mathbf{c})}\left((-1)^{\left|\mathbf{b}^{\prime}\right|} \frac{\left\langle\left\langle\lambda_{g ; I} ; \widetilde{\tau}_{\mathbf{b b}^{\prime}}\right\rangle\right\rangle}{\left(|\mathbf{b}|+\left|\mathbf{b}^{\prime}\right|-\mu_{g}(I)-m-|\mathbf{c}|\right) !} \prod_{(r, j) \in S(\mathbf{c})}\left(\begin{array}{c}
r \\
b_{r, j}^{\prime}
\end{array}\right)\right) .
\end{gathered}
$$

In particular, $|S(\mathbf{c})|=|\mathbf{c}|$ and the numerator above vanishes whenever the argument of the factorial in the denominator is negative.

Proposition 2.1. Let $g, m \in \mathbb{Z}^{\geq 0}$ with $2 g+m \geq 3$ and $I \in\left(\mathbb{Z}^{\geq 0}\right)^{g}$. There exists a collection

$$
A_{I ; \mathbf{c}}^{(g, \underline{\epsilon}} \in \mathbb{Q} \quad \text { with } \quad \underline{\epsilon} \in\left(\mathbb{Z}^{\geq 0}\right)^{m}, \mathbf{c} \in\left(\mathbb{Z}^{\geq 0}\right)^{\infty},
$$

which is invariant under the permutations of the components of $\underline{\epsilon}$ such that

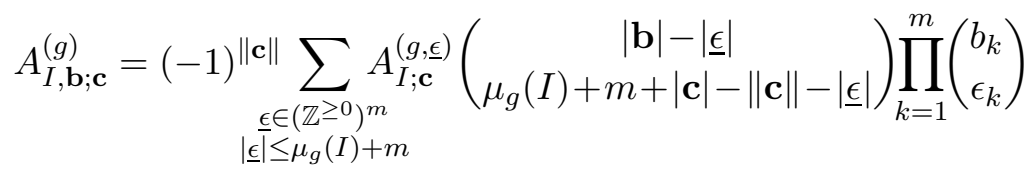

for all $\mathbf{b} \in\left(\mathbb{Z}^{\geq 0}\right)^{m}$ and $\mathbf{c} \in\left(\mathbb{Z}^{\geq 0}\right)^{\infty}$. These collections can be chosen so that there exists $C_{g} \in \mathbb{R}$ such that

$$
\left|A_{I ; \mathbf{c}}^{(g, \underline{\epsilon})}\right| \leq C_{g} 2^{\|\mathbf{c}\|}(3(g-1)+m+|\mathbf{c}|) !
$$

for all $I \in\left(\mathbb{Z}^{\geq 0}\right)^{g}, \mathbf{c} \in\left(\mathbb{Z}^{\geq 0}\right)^{\infty}, \underline{\epsilon} \in\left(\mathbb{Z}^{\geq 0}\right)^{m}$, and $m \in \mathbb{Z}^{\geq 0}$ with $2 g+m \geq 3$.

For example,

$$
\frac{\left\langle\left\langle\lambda_{g ; I} ; \widetilde{\tau}_{\mathbf{b}}\right\rangle\right\rangle}{\left(|\mathbf{b}|-\mu_{g}(I)-m\right) !}=A_{I, \mathbf{b} ; \mathbf{0}}^{(g)}=\sum_{\substack{\epsilon \in(\mathbb{Z} \geq 0)^{m} \\
|\underline{\epsilon}| \leq \mu_{g}(I)+m}} A_{I ; \underline{\mathbf{0}})}^{(g, \boldsymbol{\epsilon})}\left(\begin{array}{c}
|\mathbf{b}|-|\underline{\epsilon}| \\
\mu_{g}(I)+m-|\underline{\epsilon}|
\end{array}\right) \prod_{k=1}^{m}\left(\begin{array}{c}
b_{k} \\
\epsilon_{k}
\end{array}\right) .
$$

If $r \in \mathbb{Z}^{+}$and $e_{r} \in\left(\mathbb{Z}^{\geq 0}\right)^{\infty}$ is the $r$-th standard coordinate vector, then

$$
\begin{aligned}
& \sum_{b^{\prime}=0}^{b^{\prime}=r}\left((-1)^{b^{\prime}} \frac{\left\langle\left\langle\lambda_{g ; I} ; \widetilde{\tau}_{\mathbf{b} b^{\prime}}\right\rangle\right\rangle}{\left(|\mathbf{b}|+b^{\prime}-\mu_{g}(I)-m-1\right) !}\left(\begin{array}{c}
r \\
b^{\prime}
\end{array}\right)\right)=A_{I, \mathbf{b} ; e_{r}}^{(g)}
\end{aligned}
$$

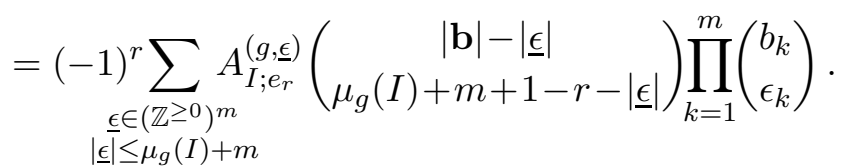

Proposition 2.1 is established in Section 5.1; all numbers $A_{I ; \mathbf{c}}^{(0, \underline{\epsilon})}$ and some numbers $A_{I ; \mathbf{c}}^{(1, \underline{\epsilon})}$ are determined in Examples 5.6 and 5.7. The bound (2.7) is used in the proof of Theorem 1. 


\section{$2.2 \quad$ Asymptotic expansions}

The second input determining the structure coefficients $\mathrm{c}_{g ; \mathbf{p}, \mathbf{b}}^{(d)}$ in Theorem $\mathrm{A}$ is the asymptotic expansion of the hypergeometric series

$$
F(w, q) \equiv \sum_{d=0}^{\infty} q^{d} \frac{w^{n d}}{\prod_{r=1}^{r=d}\left((w+r)^{n}-w^{n}\right)} \in \mathbb{Q}(w)[[q]]
$$

as $w \longrightarrow \infty$ provided by Proposition 2.2 below. By [8, 13], this hypergeometric series encodes the 1-marked genus $0 \mathrm{GW}$-invariants of $\mathbb{P}^{n-1}$.

For $n \in \mathbb{Z}^{+}$, let

$$
L(q)=(1+q)^{1 / n} \in 1+q \mathbb{Q}[[q]] .
$$

For $m, j \in \mathbb{Z}$, we define $\mathcal{H}_{m, j} \in \mathbb{Q}(u)$ recursively by

$$
\begin{aligned}
\mathcal{H}_{m, j} & \equiv 0 \quad \text { unless } \quad 0 \leq j \leq m, \quad \mathcal{H}_{0,0} \equiv 1 ; \\
\mathcal{H}_{m, j}(u) & \equiv \mathcal{H}_{m-1, j}(u)+(u-1)\left(\frac{\mathrm{d}}{\mathrm{d} u}+\frac{m-j}{n u}\right) \mathcal{H}_{m-1, j-1}(u) \quad \text { if } m \geq 1,0 \leq j \leq m .
\end{aligned}
$$

For example,

$$
\mathcal{H}_{m, 0}(u)=1, \quad \mathcal{H}_{m, 1}(u)=\left(\begin{array}{c}
m \\
2
\end{array}\right) \frac{u-1}{n u}, \quad \mathcal{H}_{m, 2}(u)=\left(\begin{array}{c}
m \\
3
\end{array}\right)\left(n+\frac{3 m-5}{4}(u-1)\right) \frac{u-1}{n^{2} u^{2}}
$$

for $m \geq 0$. Finally, we define differential operators $\mathfrak{L}_{1}, \ldots, \mathfrak{L}_{n}$ on $\mathbb{Q}[[q]]$ by

$$
D=q \frac{\mathrm{d}}{\mathrm{d} q}, \quad \mathfrak{L}_{k}=L^{n} \sum_{i=0}^{k}\left(\begin{array}{c}
n \\
i
\end{array}\right) \mathcal{H}_{n-i, k-i}\left(L^{n}\right) D^{i} .
$$

By (2.10) and (2.11), the first two operators are

$$
\begin{aligned}
& \mathfrak{L}_{1}=n L^{n} D+\frac{n-1}{2}\left(L^{n}-1\right)=n L^{n}\left\{L^{\frac{1-n}{2}} D L^{\frac{n-1}{2}}\right\}, \\
& \mathfrak{L}_{2}=\left(\begin{array}{c}
n \\
2
\end{array}\right) L^{n} D^{2}+\left(\begin{array}{c}
n-1 \\
2
\end{array}\right)\left(L^{n}-1\right) D+\frac{(n-1)(n-2)}{24 n L^{n}}\left((3 n-5) L^{n}+n+5\right)\left(L^{n}-1\right) .
\end{aligned}
$$

Proposition 2.2 ([22, Proposition 2.1]). The power series $F$ of (2.9) admits an asymptotic expansion

$$
F(w, q) \sim e^{\xi(q) w} \sum_{b=0}^{\infty} \Phi_{b}(q) w^{-b} \quad \text { as } \quad w \longrightarrow \infty,
$$

with $\xi, \Phi_{1}, \ldots \in q \mathbb{Q}[[q]]$ and $\Phi_{0} \in 1+q \mathbb{Q}[[q]]$ determined by the first-order ODEs

$$
1+D \xi=L, \quad \mathfrak{L}_{1} \Phi_{b}+\frac{1}{L} \mathfrak{L}_{2} \Phi_{b-1}+\ldots+\frac{1}{L^{n-1}} \mathfrak{L}_{n} \Phi_{b+1-n}=0,
$$

where $\Phi_{b} \equiv 0$ for $b<0$. 
For example,

$$
\begin{aligned}
& \Phi_{0}(q)=L(q)^{-(n-1) / 2}=(1+q)^{-(n-1) / 2 n} \\
& \Phi_{1}(q)=\frac{n-1}{24 n}\left(n+(n+1) L(q)^{-1}-(2 n+1) L(q)^{-(n+1)}\right) \Phi_{0}(q) .
\end{aligned}
$$

By Proposition 2.2 , for each $p \in \mathbb{Z}^{\geq 0}$ there is an asymptotic expansion

$$
\left\{1+w^{-1} D\right\}^{p} F(w, p) \sim e^{\xi(q) w} \sum_{b=0}^{\infty} \Phi_{p ; b}(q) w^{-b} \quad \text { as } \quad w \longrightarrow \infty,
$$

with $\Phi_{p ; 0} \in 1+q \mathbb{Q}[[q]]$ and $\Phi_{p ; 1}, \Phi_{p ; 2} \ldots \in q \mathbb{Q}[[q]]$ described by

$$
\Phi_{0 ; b}=\Phi_{b}, \quad \Phi_{p ;-1} \equiv 0, \quad \Phi_{p ; b}=L \Phi_{p-1 ; b}+D \Phi_{p-1 ; b-1} \quad \forall p \in \mathbb{Z}^{+}, b \in \mathbb{Z}^{\geq 0} .
$$

We set $\Phi_{p ; b}=0$ if $b<0$. For example,

$$
\Phi_{p ; 0}=L^{p} \Phi_{0}, \quad \Phi_{p ; 1}=L^{p} \Phi_{1}-\frac{(n-p) p}{2 n} L^{p-1}\left(1-L^{-n}\right) \Phi_{0}
$$

the second identity above follows by induction from the first one along with (2.10) and (2.15).

For $g \in \mathbb{Z}^{\geq 0}$, let $\left(C_{g ; n ; I}\right)_{I \in(\mathbb{Z} \geq 0)^{g}}$ be a tuple of integers such that

$$
e\left(\mathbb{E}_{g}^{*} \otimes T \mathbb{P}^{n-1}\right)=\sum_{I \in(\mathbb{Z} \geq 0)^{g}} C_{g ; n ; I} \lambda_{g ; I} H^{(n-1) g-\|I\|} \in H^{*}\left(\overline{\mathcal{M}}_{g, m} \times \mathbb{P}^{n-1}\right)
$$

for all $m \in \mathbb{Z}^{\geq 0}$ with $2 g+m \geq 3$. Let $g, m \in \mathbb{Z}^{\geq 0}$ with $2 g+m \geq 3, I \in\left(\mathbb{Z}^{\geq 0}\right)^{g}, \mathbf{c} \in\left(\mathbb{Z}^{\geq 0}\right)^{\infty}$, and $\underline{\epsilon} \in\left(\mathbb{Z}^{\geq 0}\right)^{m}$. With $A_{I ; \mathbf{c}}^{(g, \underline{\epsilon})} \in \mathbb{Q}$ as in Proposition 2.1, define

$$
\begin{gathered}
\widehat{A}_{I ;\left(c_{1}, c_{2}, \ldots\right)}^{(g, \underline{\epsilon})}=A_{I ;\left(0, c_{1}, c_{2}, \ldots\right)}^{(g, \underline{\epsilon})} \prod_{k=1}^{m} \frac{1}{\epsilon_{k} !} \\
\Phi_{I ; \mathbf{c}}^{(g, \underline{\epsilon})}(q)=\frac{(-1)^{\mu_{g}(I)+m+|\mathbf{c}|} C_{g ; n ; I} \widehat{A}_{I ; \mathbf{c}}^{(g, \underline{\epsilon}}}{\Phi_{0}(q)^{2 g-2}} \prod_{r=1}^{\infty} \frac{1}{c_{r} !}\left(\frac{\Phi_{r}(q)}{(r+1) ! \Phi_{0}(q)}\right)^{c_{r}} .
\end{gathered}
$$

Example 2.3. Using that the rank of $\mathbb{E}_{g}$ is $g$ and Euler's sequence for $\mathbb{P}^{n-1}$, we obtain

$$
C_{0 ; n ;()}=1, \quad C_{1 ; n ;(0)}=n, \quad C_{1 ; n ;(1)}=-\frac{(n-1) n}{2}
$$

For $\mathbf{0} \in\left(\mathbb{Z}^{\geq 0}\right)^{m}$, these statements and Example 5.6 give

$$
\begin{aligned}
& \Phi_{() ; \mathbf{c}}^{(0, \mathbf{0})}(q)=(-1)^{m-3+|\mathbf{c}|}(m-3-|\mathbf{c}|) ! \Phi_{0}(q)^{2} \prod_{r=1}^{\infty} \frac{1}{c_{r} !}\left(\frac{\Phi_{r}(q)}{(r+1) ! \Phi_{0}(q)}\right)^{c_{r}} \\
& \Phi_{(1) ; \mathbf{c}}^{(1, \mathbf{0})}(q)=(-1)^{m+|\mathbf{c}|} \frac{(n-1) n(m-1-|\mathbf{c}|) !}{48} \prod_{r=1}^{\infty} \frac{1}{c_{r} !}\left(\frac{\Phi_{r}(q)}{(r+1) ! \Phi_{0}(q)}\right)^{c_{r}} .
\end{aligned}
$$


All other power series $\Phi_{I ; \mathbf{c}}^{(g, \underline{\epsilon})}$ with $g=0$ and $(g, I)=(1,(1))$ vanish. The first expression above equals $\Phi_{m-3, \mathbf{c}}(q)$ in $[22,(2.30)]$. For $\underline{\epsilon} \in\{0,1\}^{m}$, Example 5.7 gives

$$
\Phi_{(0) ; \mathbf{0}}^{(1, \underline{\epsilon})}(q)=(-1)^{m} \frac{n(m-|\underline{\epsilon}|) !}{24} \cdot \begin{cases}0, & \text { if }|\underline{\epsilon}|=0 \\ 1, & \text { if }|\underline{\epsilon}|=1 \\ -(|\underline{\epsilon}|-2) !, & \text { if }|\epsilon| \geq 2\end{cases}
$$

If in addition $r \in \mathbb{Z}^{+}$and $e_{r} \in\left(\mathbb{Z}^{\geq 0}\right)^{\infty}$ is the $r$-th standard coordinate vector, then

$$
\Phi_{(0) ; e_{r}}^{(1, \underline{\epsilon})}(q)=(-1)^{m+1} \frac{n(m-|\underline{\epsilon}|) !}{24(r+1) !} \frac{\Phi_{r}(q)}{\Phi_{0}(q)} \cdot \begin{cases}(r+1), & \text { if }|\underline{\epsilon}|=0 \\ (m-1-r), & \text { if }|\underline{\epsilon}|=1 \\ -(|\underline{\epsilon}|-2) !((|\underline{\epsilon}|-1) r+m), & \text { if }|\epsilon| \geq 2\end{cases}
$$

The power series $\Phi_{I ; \mathbf{c}}^{(1, \underline{\epsilon}}$ with $\underline{\epsilon} \notin\{0,1\}^{m}$ for any $m \in \mathbb{Z}^{+}$vanish.

\subsection{The structure coefficients}

We now describe the coefficients $\mathrm{c}_{g ; \mathbf{p}, \mathbf{b}}^{(d)}$ in (2.3) explicitly in two ways. The first description provides a closed formula for these coefficients as sums over connected trivalent $N$-marked genus $g$ graphs; see (2.29). The second description provides a recursive definition of these coefficients which reduces the value of $3 g+N$ (or equivalently of the dimension of $\overline{\mathcal{M}}_{g, N}$ ) with the base case provided by (2.32), when this value is 2 ; see (2.33). We also show that these coefficients satisfy

$$
\mathrm{c}_{g ; \mathbf{p}, \mathbf{b}}^{(d)} \neq 0 \quad \Longrightarrow \quad|\mathbf{b}| \leq 3(g-1)+N, \quad|\mathbf{p}|-|\mathbf{b}|+n d=(n-4)(g-1)+(n-2) N .
$$

It is fairly straightforward to see that the two descriptions are equivalent. This also follows from the two variations of the main localization computation in Sections 4.2 and 4.3 . For a ring $R$, $\Phi \in R[[q]]$, and $d \in \mathbb{Z}$, let

$$
\llbracket \Phi \rrbracket_{q ; d} \in R
$$

denote the coefficient of $q^{d}$ (with $\llbracket \Phi \rrbracket_{q ; d} \equiv 0$ if $d<0$ ).

Let $S$ be a finite set. An $S$-marked graph is a tuple

$$
\Gamma \equiv\left(\mathfrak{g}: \operatorname{Ver} \longrightarrow \mathbb{Z}^{\geq 0}, \eta: S \sqcup \mathrm{Fl} \longrightarrow \operatorname{Ver}, \mathrm{Edg}\right),
$$

where Ver and $\mathrm{Fl}$ are finite sets (of vertices and flags, respectively) and Edg is a partition of $\mathrm{Fl}$ into subsets $e$ with $|e|=2$. For $N \in \mathbb{Z}^{\geq 0}$, an $N$-marked graph is an [N]-marked graph. Figure 1 depicts some 2-marked graphs $\Gamma$, representing each vertex of $\Gamma$ by a dot and each edge by a curve between its vertices. The number next to a vertex $v$, if any, is $\mathfrak{g}(v)$; we omit it if $\mathfrak{g}(v)=0$. The elements of the set $[N]=[2]$ are shown in bold face and are linked by line segments to their images under $\eta$.

An equivalence between an $S$-marked graph as in (2.23) and another $S$-marked graph

$$
\Gamma^{\prime} \equiv\left(\mathfrak{g}^{\prime}: \operatorname{Ver}^{\prime} \longrightarrow \mathbb{Z}^{\geq 0}, \eta^{\prime}: S \sqcup \mathrm{Fl}^{\prime} \longrightarrow \operatorname{Ver}^{\prime}, \mathrm{Edg}^{\prime}\right)
$$

is a pair of bijections $h_{\mathrm{Ver}}: \mathrm{Ver} \longrightarrow \mathrm{Ver}^{\prime}$ and $h_{\mathrm{Fl}}: \mathrm{Fl} \longrightarrow \mathrm{Fl}^{\prime}$ such that

$$
\mathfrak{g}=\mathfrak{g}^{\prime} \circ h_{\mathrm{Ver}},\left.\quad h_{\mathrm{Ver}} \circ \eta\right|_{S}=\left.\eta^{\prime}\right|_{S},\left.\quad h_{\mathrm{Ver}} \circ \eta\right|_{\mathrm{Fl}}=\eta^{\prime} \circ h_{\mathrm{Fl}}, \quad h_{\mathrm{Fl}}(e) \in \mathrm{Edg}^{\prime} \forall e \in \mathrm{Edg} .
$$



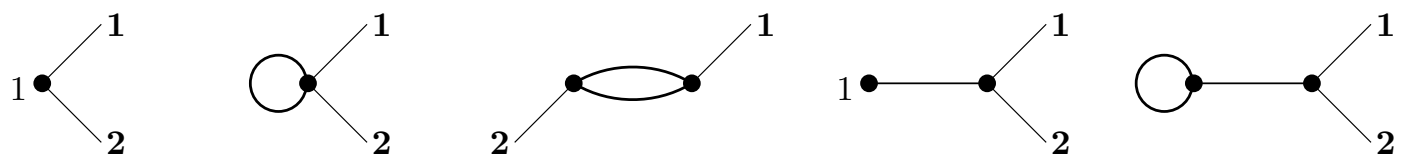

Figure 1: The trivalent 2-marked genus 1 graphs

We denote by $\operatorname{Aut}(\Gamma)$ the group of automorphisms, i.e. self-equivalences, of $\Gamma$. For example, $\operatorname{Aut}(\Gamma)=2$ for the second and third graphs in Figure 1.

For $\Gamma$ as in (2.23) and $f \in \mathrm{Fl}$, we denote by $e_{f} \in \operatorname{Edg}$ the unique element of Edg containing $f$. For each $v \in$ Ver, let

$$
\begin{aligned}
g_{v}=\mathfrak{g}(v), \quad S_{v} & =S \cap \eta^{-1}(v), \quad \mathrm{Fl}_{v}(\Gamma)=\mathrm{Fl} \cap \eta^{-1}(v), \quad \overline{\mathrm{Fl}}_{v}(\Gamma)=\eta^{-1}(v) \subset S \sqcup \mathrm{Fl}, \\
\operatorname{val}_{\Gamma}(v) & =2\left(g_{v}-1\right)+\left|\overline{\mathrm{Fl}}_{v}(\Gamma)\right|, \quad m_{v}(\Gamma)=3\left(g_{v}-1\right)+\left|\overline{\mathrm{Fl}}_{v}(\Gamma)\right| .
\end{aligned}
$$

A vertex $v \in \operatorname{Ver}$ of $\Gamma$ is trivalent if $\operatorname{val}_{\Gamma}(v)>0$. The graph $\Gamma$ is trivalent if all its vertices are trivalent.

A graph $\Gamma$ as in (2.23) is connected if for all $v, v^{\prime} \in$ Ver distinct there exist

$$
\begin{gathered}
m \in \mathbb{Z}^{+}, f_{1}^{-}, f_{1}^{+}, \ldots, f_{m}^{-}, f_{m}^{+} \in \mathrm{Fl} \quad \text { s.t. } \\
\eta\left(f_{1}^{-}\right)=v, \eta\left(f_{m}^{+}\right)=v^{\prime}, \eta\left(f_{i}^{+}\right)=\eta\left(f_{i+1}^{-}\right) \forall i \in[m-1], e_{f_{i}^{-}}=e_{f_{i}^{+}} \forall i \in[m] .
\end{gathered}
$$

For a connected graph $\Gamma$ as in (2.23), we define

$$
g_{\Gamma} \equiv 1-|\operatorname{Ver}|+|\operatorname{Edg}| \in \mathbb{Z}^{\geq 0} \quad \text { and } \quad \mathfrak{a}(\Gamma) \equiv g_{\Gamma}+\sum_{v \in \operatorname{Ver}} \mathfrak{g}(v)
$$

to be the arithmetic genus of the underlying graph without the map $\mathfrak{g}$ and the arithmetic genus of the graph $\Gamma$ itself, respectively. For such a graph,

$$
\sum_{v \in \text { Ver }}\left|\overline{\mathrm{Fl}}_{v}(\Gamma)\right|=2|\operatorname{Edg}|+|S|, \quad \sum_{v \in \text { Ver }} m_{v}(\Gamma)+|\operatorname{Edg}|=3(\mathfrak{a}(\Gamma)-1)+|S|
$$

the first equality above does not depend on $\Gamma$ being connected. For $g, N \in \mathbb{Z}^{\geq 0}$, we denote by $\mathcal{A}_{g, N}$ the set of (equivalence classes of) connected trivalent $N$-marked genus $g$ graphs. The two elements of $\mathcal{A}_{1,1}$ and the five elements of $\mathcal{A}_{1,2}$ are shown in Figures 2 and 1 , respectively.

Let $\Gamma$ be an $N$-marked graph as in (2.23) with $S=[N]$. For $\mathbf{b} \in\left(\mathbb{Z}^{\geq 0}\right)^{\overline{\mathrm{Fl}}_{v}(\Gamma)}$ and $f \in \overline{\mathrm{Fl}}_{v}(\Gamma)$, we denote by $b_{f} \in \mathbb{Z}^{\geq 0}$ the component of $\mathbf{b}$ corresponding to $f$. For

$$
\mathbf{b} \in\left(\mathbb{Z}^{\geq 0}\right)^{\overline{\mathrm{Fl}}(\Gamma)}, \quad \mathbf{c} \in \prod_{v \in \operatorname{Ver}}\left(\mathbb{Z}^{\geq 0}\right)^{\infty}, \quad \text { and } \quad \mathbf{I} \in \prod_{v \in \operatorname{Ver}}\left(\mathbb{Z}^{\geq 0}\right)^{g_{v}}
$$

we similarly denote their respective components by

$$
b_{f} \in \mathbb{Z}^{\geq 0} \text { for } f \in \overline{\mathrm{Fl}}(\Gamma) \quad \text { and } \quad \mathbf{b}_{v} \in\left(\mathbb{Z}^{\geq 0}\right)^{\overline{\mathrm{Fl}}_{v}(\Gamma)}, \mathbf{c}_{v} \in\left(\mathbb{Z}^{\geq 0}\right)^{\infty}, I_{v} \in\left(\mathbb{Z}^{\geq 0}\right)^{g_{v}} \text { for } v \in \text { Ver }
$$


Define

$$
\begin{array}{r}
\widetilde{\mathcal{A}}^{\star}(\Gamma)=\left\{(\mathbf{b}, \underline{\epsilon}, \mathbf{c}, \mathbf{I}) \in\left(\mathbb{Z}^{\geq 0}\right)^{\overline{\mathrm{Fl}}(\Gamma)} \times\left(\mathbb{Z}^{\geq 0}\right)^{\overline{\mathrm{Fl}}(\Gamma)} \times \prod_{v \in \operatorname{Ver}}\left(\mathbb{Z}^{\geq 0}\right)^{\infty} \times \prod_{v \in \text { Ver }}\left(\mathbb{Z}^{\geq 0}\right)^{g_{v}}:\right. \\
\left.\left|\mathbf{b}_{v}\right|+\left|\underline{\epsilon}_{v}\right|+\left\|\mathbf{c}_{v}\right\|=\mu_{g_{v}}\left(I_{v}\right)+\left|\overline{\mathrm{Fl}}_{v}(\Gamma)\right| \forall v \in \text { Ver }\right\} .
\end{array}
$$

Choose a labeling of the flags so that each edge $e$ of $\Gamma$ contains flags with opposite signs. For each vertex $v \in$ Ver of $\Gamma$, denote by

$$
\mathrm{Fl}_{v}^{ \pm}(\Gamma) \subset \mathrm{Fl}_{v}(\Gamma)
$$

the subset of positive/negative flags at $v$. For $p \in \llbracket n \rrbracket$, let

$$
\widehat{p}=n-1-p \in \llbracket n \rrbracket .
$$

For $d \in \mathbb{Z}^{\geq 0}, \mathbf{p} \in\left\lfloor n \rrbracket^{N}\right.$, and $\mathbf{b} \in\left(\mathbb{Z}^{\geq 0}\right)^{N}$, we define

$$
\mathcal{S}_{\Gamma}(d, \mathbf{p}, \mathbf{b}) \subset\left(\mathbb{Z}^{\geq 0}\right)^{\mathrm{Ver}} \times \llbracket n \rrbracket^{\operatorname{Edg}} \times\left(\mathbb{Z}^{\geq 0}\right)^{\mathrm{Edg}}
$$

to be the subset of triples $\left(\mathbf{d}, \mathbf{p}^{\prime}, \mathbf{b}^{\prime}\right)$ such that

$$
\begin{aligned}
& \sum_{v \in \operatorname{Ver}} d_{v}=d \\
& \sum_{s \in S_{v}}\left(\widehat{p}_{s}+b_{s}\right)+\sum_{f \in \mathrm{Fl}_{v}^{-}(\Gamma)}\left(p_{e_{f}}^{\prime}+b_{e_{f}}^{\prime}\right)+\sum_{f \in \mathrm{Fl}_{v}^{+}(\Gamma)}\left(\widehat{p}_{e_{f}}^{\prime}-1-b_{e_{f}}^{\prime}\right) \\
& =(n-4)\left(1-g_{v}\right)+\left|\overline{\mathrm{Fl}}_{v}(\Gamma)\right|+n d_{v} \quad \forall v \in \text { Ver. }
\end{aligned}
$$

By (2.24) and the first statement in (2.25), this set is empty unless the second property on the right-hand side of (2.22) with $g=\mathfrak{a}(\Gamma)$ holds.

For $(\mathbf{p}, \mathbf{b}) \in \llbracket n \rrbracket^{N} \times\left(\mathbb{Z}^{\geq 0}\right)^{N}$ and $d \in \mathbb{Z}^{\geq 0}$, let

$$
\begin{gathered}
\mathrm{c}_{g ; \mathbf{p}, \mathbf{b}}^{(d)}=\sum_{\Gamma \in \mathcal{A}_{g, N}} \frac{1}{|\operatorname{Aut}(\Gamma)|} \sum_{\substack{\left(\mathbf{d}, \mathbf{p}^{\prime}, \mathbf{b}^{\prime}\right) \in \mathcal{S}_{\Gamma}(d, \mathbf{p}, \mathbf{b}) \\
\left(\mathbf{b}^{\prime \prime}, \epsilon, \mathbf{c}, \mathbf{I}\right) \in \widetilde{\mathcal{A}}^{\star}(\Gamma)}}(-1)^{|\mathbf{b}|+\left|\mathbf{b}^{\prime}\right|} \prod_{v \in \mathrm{Ver}} \\
\left\|\Phi_{I_{v} ; \mathbf{c}_{v}}^{\left(g_{v}, \epsilon_{v}\right)}(q) \prod_{s \in S_{v}} \frac{\Phi_{\widehat{p}_{s} ; b_{s}^{\prime \prime}+\epsilon_{s}-b_{s}}(q)}{b_{s}^{\prime \prime} ! \Phi_{0}(q)} \prod_{f \in \mathrm{Fl}_{v}^{-}(\Gamma)} \frac{\Phi_{p_{e_{f}}^{\prime} ; b_{f}^{\prime \prime}+\epsilon_{f}-b_{e_{f}}^{\prime}}(q)}{b_{f}^{\prime \prime} ! \Phi_{0}(q)} \prod_{f \in \mathrm{Fl}_{v}^{+}(\Gamma)} \frac{\Phi_{\widehat{p}_{e_{f}}^{\prime} ; b_{f}^{\prime \prime}+\epsilon_{f}+1+b_{e_{f}}^{\prime}}(q)}{b_{f}^{\prime \prime} ! \Phi_{0}(q)}\right\|_{q ; d_{v}} .
\end{gathered}
$$

Since

$$
\sum_{s \in S_{v}} b_{s} \leq \sum_{s \in S_{v}}\left(b_{s}^{\prime \prime}+\epsilon_{s}\right) \leq 3\left(g_{v}-1\right)+\left|\overline{\mathrm{Fl}}_{v}(\Gamma)\right| \equiv m_{v}(\Gamma)
$$

if the corresponding factor on the second line of (2.29) is nonzero, the second identity in (2.25) implies the first property in (2.22).

We next give a recursive description of the coefficients $\mathrm{c}_{g ; \mathbf{p}, \mathbf{b}}^{(d, 0)} \equiv \mathrm{c}_{g ; \mathbf{p}, \mathbf{b}}^{(d)}$ appearing in Theorem $\mathrm{A}$. For $N \in \mathbb{Z}^{\geq 0}$, a tuple $\mathbf{p} \equiv\left(p_{s}\right)_{s \in[N]}$ of integers, and $S \subset[N]$, let $\left.\mathbf{p}\right|_{S}$ be the $S$-tuple consisting of the elements of $\mathbf{p}$ indexed by $S$ and

$$
|\mathbf{p}|_{S} \equiv|\mathbf{p}|_{S} \mid \equiv \sum_{s \in S} p_{s}
$$


For $m \in \mathbb{Z}^{\geq 0}$, let $\mathcal{P}_{g, N}^{(m)}$ denote the collection of tuples $\left(g^{\prime},\left(g_{i}, S_{i}, N_{i}\right)_{i \in[m]}\right)$ such that

$$
\begin{gathered}
g^{\prime} \in \mathbb{Z}^{\geq 0}, \quad g_{i} \in \mathbb{Z}^{\geq 0}, N_{i} \in \mathbb{Z}^{+} \forall i \in[m], \quad[N]=\bigsqcup_{i=1}^{i=m} S_{i}, \quad 2 g^{\prime}+\sum_{i=1}^{i=m} N_{i} \geq 3, \\
(0,\{N\}, 1) \in\left\{\left(g_{i}, S_{i}, N_{i}\right): i \in[m]\right\}, \quad g_{i}+\left|S_{i}\right|+\left|N_{i}\right| \geq 2 \quad \forall i \in[m], \quad g^{\prime}+\sum_{i=1}^{i=m}\left(g_{i}+N_{i}\right)=g+m .
\end{gathered}
$$

We write elements of $\mathcal{P}_{g, N}^{(m)}$ as tuples $\left(g^{\prime}, \mathbf{g}, \mathbf{S}, \mathbf{N}\right)$ and denote the components of the $m$-tuples $\mathbf{g}, \mathbf{S}$, and $\mathbf{N}$ by $g_{i}, S_{i}$, and $N_{i}$, respectively. We note that

$$
3 g_{i}+\left|S_{i}\right|+N_{i}<3 g+N \quad \forall i \in[m]
$$

if $\left(g^{\prime},\left(g_{i}, S_{i}, N_{i}\right)_{i \in[m]}\right)$ is an element of $\mathcal{P}_{g, N}^{(m)}$.

Remark 2.4. Let $\Gamma$ be an $N$-marked genus $g$ graph as in (2.23) with $S=[N]$ and $v \equiv \eta(N)$ be the vertex to which the last marked point is attached. Breaking $\Gamma$ at $v$ and replacing the flags at $v$ with marked points, we obtain $m$ connected strands similarly to Figure 4 on page 30 for some $m \in \mathbb{Z}^{+}$. Each of the strands is of some genus $g_{i}$, carries the subset $S_{i} \subset[N]$ of the original marked points, and $N_{i} \in \mathbb{Z}^{+}$additional marked points arising from the flags at $v$. Then,

$$
\left(\mathfrak{g}(v),\left(g_{i}, S_{i}, N_{i}\right)_{i \in[m]}\right) \in \mathcal{P}_{g, N}^{(m)} .
$$

Furthermore, every element of $\Gamma$ describes a type of collections of strands obtained by breaking an $N$-marked genus $g$ graph $\Gamma$ at the vertex $v \equiv \eta(N)$ to which the last marked point is attached.

For $m, g^{\prime}$, and $\mathbf{N}$ as above, define

$$
\begin{aligned}
\widetilde{\mathcal{A}}_{g^{\prime}, \mathbf{N}}^{\star}=\left\{(\mathbf{b}, \underline{\epsilon}, \mathbf{c}, I) \in \prod_{i=1}^{i=m}\left(\mathbb{Z}^{\geq 0}\right)^{N_{i}} \times \prod_{i=1}^{i=m}\left(\mathbb{Z}^{\geq 0}\right)^{N_{i}} \times\left(\mathbb{Z}^{\geq 0}\right)^{\infty} \times\left(\mathbb{Z}^{\geq 0}\right)^{g^{\prime}}:\right. \\
\left.|\mathbf{b}|+|\underline{\epsilon}|+\|\mathbf{c}\|=\mu_{g^{\prime}}(I)+|\mathbf{N}|\right\} .
\end{aligned}
$$

If in addition $d, t \in \mathbb{Z}$, let

$$
\mathcal{S}_{g^{\prime}, \mathbf{N}}(d, t)=\left\{(\mathbf{p}, \mathbf{b}) \in \prod_{i=1}^{i=m}\left\lfloor n \rrbracket^{N_{i}} \times \prod_{i=1}^{i=m} \mathbb{Z}^{N_{i}}:|\mathbf{p}|-|\mathbf{b}|=(n-4)\left(1-g^{\prime}\right)+2|\mathbf{N}|+n(d+t)\right\} ;\right.
$$

the number $|\mathbf{b}|$ above denotes the sum of the three components of $\mathbf{b}$ (not of their absolute values). For tuples $\mathbf{p}$ and $\mathbf{b}$ as above and $i \in[m]$, we denote by $\mathbf{p}_{i} \in \llbracket n \rrbracket^{N_{i}}$ and $\mathbf{b}_{i} \in \mathbb{Z}^{N_{i}}$ the $i$-th components of $\mathbf{p}$ and $\mathbf{b}$. If in addition $f \in\left[N_{i}\right]$, let $p_{i ; f} \in\left\lfloor n \rrbracket\right.$ and $b_{i ; f} \in \mathbb{Z}$ denote the $f$-th components of $\mathbf{p}_{i}$ and $\mathbf{b}_{i}$, respectively.

For any $p, p^{\prime} \in\left\lfloor n \rrbracket\right.$ and $b, b^{\prime}, d, t \in \mathbb{Z}$, let

$$
\mathrm{c}_{0 ;\left(p, p^{\prime}\right),\left(b, b^{\prime}\right)}^{(d, t)}= \begin{cases}(-1)^{b}, & \text { if } b \geq 0, b+b^{\prime}=-1, d, t=0, p+p^{\prime}=n-1 \\ 0, & \text { otherwise }\end{cases}
$$


For all $g \in \mathbb{Z}^{\geq 0}, N \in \mathbb{Z}^{+}$with $2 g+N \geq 3, N$-tuples $\mathbf{p} \in \llbracket n \rrbracket^{N}$ and $\mathbf{b} \in\left(\mathbb{Z}^{\geq 0}\right)^{N}$, and $d, t \in \mathbb{Z}^{\geq 0}$, we inductively define

$$
\begin{aligned}
& \mathrm{c}_{g ; \mathbf{p}, \mathbf{b}}^{(d, t)}=\sum_{\substack{m, d^{\prime} \in \mathbb{Z} \geq 0 \\
t^{\prime} \in \mathbb{Z}}} \frac{1}{m !} \sum_{\substack{\left(g^{\prime}, \mathbf{g}, \mathbf{S}, \mathbf{N}\right) \in \mathcal{P}_{g, N}^{(m)} \\
\left(\mathbf{b}^{\prime \prime}, \underline{\epsilon}, \mathbf{c}, I\right) \in \widetilde{\mathcal{A}}_{g^{\prime}, \mathbf{N}}^{\star} \\
\left(\mathbf{p}^{\prime}, \mathbf{b}^{\prime}\right) \in \mathcal{S}_{g^{\prime}, \mathbf{N}}\left(d^{\prime}, t^{\prime}\right)}} \sum_{\substack{\mathbf{d}, \mathbf{t} \in(\mathbb{Z} \geq 0)^{m} \\
\mid}} \prod_{i=1-d^{\prime},|\mathbf{t}|=t-t^{\prime}} \prod_{i=m}^{i=m} \frac{\mathrm{c}_{g_{i} ; \mathbf{p} \mid S_{i}}^{\left(d_{i}, \mathbf{p}_{i}\right)} \mathbf{p}_{i}^{\prime},\left.\mathbf{b}\right|_{S_{i}} \mathbf{b}_{i}^{\prime}}{N_{i} !} \\
& \left.\times \llbracket \Phi_{I ; \mathbf{c}}^{\left(g^{\prime}, \underline{\epsilon}\right)}(q) \prod_{i=1}^{i=m} \prod_{f \in\left[N_{i}\right]} \frac{\Phi_{p_{i ; f}^{\prime} ; b_{i ; f}^{\prime \prime}+\epsilon_{i ; f}+1+b_{i ; f}^{\prime}(q)}}{b_{i ; f}^{\prime \prime} ! \Phi_{0}(q)} \rrbracket_{q ; d^{\prime}}\right),
\end{aligned}
$$

with $\mathrm{c}_{g_{i} ;\left.\mathbf{p}\right|_{S_{i}} \mathbf{p}_{i}^{\prime},\left.\mathbf{b}\right|_{S_{i}} \mathbf{b}_{i}^{\prime}}^{\left(d_{i}, t_{i}\right)} \equiv 0$ if $b_{i ; f}^{\prime}<0$ for some $f \in\left[N_{i}\right]$ and $2 g_{i}+\left|S_{i}\right|+N_{i} \geq 3$ (if the last sum is 2, $\mathrm{c}_{g_{i} ;\left.\mathbf{p}\right|_{S_{i}} \mathbf{p}_{i}^{\prime},\left.\mathbf{b}\right|_{S_{i}} \mathbf{b}_{i}^{\prime}}^{\left(d_{i}, t_{i}\right)}$ is given by $(\underline{2.32})$ ).

If the summand in (2.33) above does not vanish, then

$$
-b_{i ; f}^{\prime} \leq b_{i ; f}^{\prime \prime}+\epsilon_{i ; f}+1 \quad \forall f \in\left[N_{i}\right], i \in[m] \quad \Longrightarrow \quad-\left|\mathbf{b}^{\prime}\right| \leq 3 g^{\prime}-3+2|\mathbf{N}| ;
$$

the last implication makes use of the condition $\left(\mathbf{b}^{\prime \prime}, \underline{\epsilon}, \mathbf{c}, I\right) \in \widetilde{\mathcal{A}}_{g^{\prime}, \mathbf{N}}^{\star}$. By induction, the non-vanishing coefficients $\mathrm{c}_{g ; \mathbf{p}, \mathbf{b}}^{(d, t)}$ thus satisfy the bound in (2.22). Furthermore,

$$
\mathrm{c}_{g ; \mathbf{p}, \mathbf{b}}^{(d, t)} \neq 0 \Longrightarrow|\mathbf{p}|-|\mathbf{b}|+n(d+t)=(n-4)(g-1)+(n-2) N .
$$

Thus, the coefficients $\mathrm{c}_{g ; \mathbf{p}, \mathbf{b}}^{(d, t)} \equiv \mathrm{c}_{g ; \mathbf{p}, \mathbf{b}}^{(d, 0)}$ defined by (2.32) and (2.33) satisfy (2.22).

Since $\Phi_{I ; \mathbf{c}}^{\left(g^{\prime}, \underline{\epsilon}\right)} \in q \mathbb{Q}[[q]]$ unless $\mathbf{c}=\mathbf{0}$ and $\Phi_{p^{\prime} ; b^{\prime}} \in q \mathbb{Q}[[q]]$ unless $b^{\prime}=0$, all nonzero contributions in the $d=0$ case of $(2.33)$ arise from the elements $\left(g^{\prime}, \mathbf{g}, \mathbf{S}, \mathbf{N}\right)$ of $\mathcal{P}_{g, N}^{(m)}$ so that $g_{i}=0$ and $\left|S_{i}\right|,\left|N_{i}\right|=1$ for every $i \in[m]$. These conditions imply that $g^{\prime}=g$ and $m=N$. By the first statement after (2.16) and (2.20),

$$
\llbracket \Phi_{p^{\prime} ; 0} \rrbracket_{q ; 0}=1 \forall p^{\prime} \in \llbracket n \rrbracket, \quad \llbracket \Phi_{I ; \mathbf{0}}^{(g, \underline{\epsilon})}(q) \rrbracket_{q ; 0}=(-1)^{\mu_{g}(I)+N} C_{g ; n ; I} \widehat{A}_{I ; \mathbf{0}}^{(g, \underline{\epsilon})} \forall I \in\left(\mathbb{Z}^{\geq 0}\right)^{g}, \underline{\epsilon} \in\left(\mathbb{Z}^{\geq 0}\right)^{N} .
$$

Combining these observations with (2.33) and (2.32), we obtain

$$
\begin{aligned}
\mathrm{c}_{g ; \mathbf{p}, \mathbf{b}}^{(0, t)}= & \sum_{\substack{\left.\left(\mathbf{b}^{\prime \prime}, \underline{\epsilon}, \mathbf{0}, I\right) \in \widetilde{\mathcal{A}}_{g,[N]}^{\star}\right] \\
\left|\mathbf{p}^{\prime}\right|=(n-1)(1-g)+n t+\|I\|}}(-1)^{\mu_{g}(I)+N} C_{g ; n ; I} \widehat{A}_{I ; \mathbf{0}}^{(g, \underline{\epsilon})} \prod_{s=1}^{s=N}(-1)^{b_{s}} \frac{\delta_{\widehat{p}_{s}, p_{s}^{\prime}} \delta_{b_{s}, b_{s}^{\prime \prime}+\epsilon_{s}}}{b_{s}^{\prime \prime} !} \\
= & \sum_{\substack{I \in(\mathbb{Z} \geq 0)^{g} \\
\mu_{g}(I)=|\mathbf{b}|-N}} \delta_{|\mathbf{p}|+\|I\|+n t,(n-1)(N-1+g)} \sum_{\substack{\epsilon \in(\mathbb{Z} \geq 0)^{N} \\
C_{g ; n ; I}}} A_{I ; \mathbf{0})}^{(g, \underline{\epsilon})} \prod_{s=1}^{s=N} \frac{1}{b_{s} !}\left(\begin{array}{c}
b_{s} \\
\epsilon_{s}
\end{array}\right) \\
= & \delta_{|\mathbf{p}|-|\mathbf{b}|+n t,(n-4)(g-1)+(n-2) N} \sum_{\substack{I \in(\mathbb{Z} \geq 0)^{g} \\
|\mathbf{b}|+\|I\|=3(g-1)+N}} C_{g ; n ; I}\left\langle\left\langle\lambda_{g ; I} ; \tau_{\mathbf{b}}\right\rangle\right)
\end{aligned}
$$

where $\delta_{a, b}$ is the Kronecker delta function (equals 1 if $a=b$ and 0 otherwise); the last equality above follows from (2.8). The $t=0$ case of the above statement can also be obtained from (2.29). 
The coefficients $\mathrm{c}_{g ; \mathbf{p}, \mathbf{b}}^{(d)}$ must be invariant under the permutations of $[N]$ (same permutations in the components of $\mathbf{p}$ and $\mathbf{b}$ ). This is not apparent from either of the above two descriptions of these coefficients, even in the extremal cases; thus, this is a consequence of the proofs of Theorem $\mathrm{A}$ in Section 4 .

\subsection{Proof of Theorem 0}

The statement of Theorem 0 is equivalent to

$$
\sum_{p=0}^{n-1}\left\langle\frac{H^{n-1-p}}{\hbar-\psi_{1}}\right\rangle_{1, d}^{\mathbb{P}^{n-1}} H^{p}=\frac{n}{24} \frac{(H+d \hbar)^{n-1}}{\hbar^{2} \prod_{r=1}^{d}(H+r \hbar)^{n}}-\frac{(n-1) n}{48} \frac{(H+d \hbar)^{n-2}}{\hbar \prod_{r=1}^{d}(H+r \hbar)^{n}},
$$

with the identity holding modulo $H^{n}$ and as a power series in $\hbar^{-1}$. We deduce this identity from Theorem A below.

By the defining property of the cohomology pushforward, the generating function (2.1) with $(g, N)=(1,1)$ is given by

$$
Z^{(1)}(\hbar, H, q)=\sum_{d=0}^{\infty}\left(q^{d} \sum_{p=0}^{n-1}\left\langle\frac{H^{n-1-p}}{\hbar-\psi_{1}}\right\rangle_{1, d}^{\mathbb{P}^{n-1}} H^{p}\right) \in H^{*}\left(\mathbb{P}^{n-1}\right)\left[\hbar^{-1}\right][[q]] .
$$

By (2.3),

$$
Z^{(1)}(\hbar, H, q)=\sum_{p \in \llbracket n \rrbracket b \in\{0,1\}} \sum_{d=0}^{\infty} c_{1 ; p, b}^{(d)} q^{d} \hbar^{-b} \Delta_{p}(\hbar, H, q)
$$

with the coefficients $\mathrm{c}_{1 ; p, b}^{(d)} \in \mathbb{Q}$ determined by either the closed formula (2.29) or the recursion (2.33). In order to compute these coefficients, we will make use of

$$
\begin{gathered}
\Phi_{() ; \mathbf{0}}^{\left(0,\left(\mathbf{0}_{3}\right)\right)}=\Phi_{0}^{2}, \quad \Phi_{(1) ; \mathbf{0}}^{(1,(0))}=-\frac{(n-1) n}{48}, \quad \Phi_{(0) ; \mathbf{0}}^{(1,(0))}=0, \quad \Phi_{(0) ; \mathbf{0}}^{(1,(1))}=-\frac{n}{24}, \quad \Phi_{(0) ; e_{1}}^{(1,(0))}=\frac{n}{24} \frac{\Phi_{1}}{\Phi_{0}}, \\
\sum_{p=0}^{n-1} \frac{\Phi_{p ; 0}}{\Phi_{0}} \frac{\Phi_{\widehat{p} ; 1}}{\Phi_{0}}=n L^{n-1} \frac{\Phi_{1}}{\Phi_{0}}-\frac{n^{2}-1}{12} L^{n-2}\left(1-L^{-n}\right),
\end{gathered}
$$

where $\mathbf{0}_{3} \in\left(\mathbb{Z}^{\geq 0}\right)^{3}$ and $\mathbf{0} \in\left(\mathbb{Z}^{\geq 0}\right)^{\infty}$ are the zero vectors and $e_{1}=(1,0, \ldots) \in \mathbb{Z}^{\infty}$; see Example 2.3 and (2.17).

The set $\mathcal{A}_{1,1}$ of connected trivalent 1 -marked genus 1 graphs consists of two elements, $\Gamma_{0}$ and $\Gamma_{1}$; they are depicted in Figure 2 along with the corresponding collections (2.26). The associated collections (2.27) are

$$
\begin{aligned}
& \mathcal{S}_{\Gamma_{0}}(d, p, b)= \begin{cases}\{(d, \cdot, \cdot)\}, & \text { if } d=0, p=n-2+b ; \\
\emptyset, & \text { otherwise; }\end{cases} \\
& \mathcal{S}_{\Gamma_{1}}(d, p, b)= \begin{cases}\{d\} \times \llbracket n \rrbracket \times \mathbb{Z} \geq 0, & \text { if } d=0, p=n-2+b ; \\
\emptyset, & \text { otherwise. }\end{cases}
\end{aligned}
$$




$$
\begin{aligned}
& \left|\operatorname{Aut}\left(\Gamma_{0}\right)\right|=1 \\
& \mathbf{1} \text { Ver }=\{v\} \\
& \begin{array}{l}
S_{v}=\{1\} \\
\overline{\mathrm{Fl}}_{v}\left(\Gamma_{0}\right)=\{1\}
\end{array} \\
& \begin{array}{l}
S_{v}=\{1\} \\
\overline{\mathrm{Fl}}_{v}\left(\Gamma_{0}\right)=\{1\}
\end{array} \\
& \text { Ver }=\{v\} \\
& S_{v}=\{1\} \\
& \overline{\mathrm{Fl}}_{v}\left(\Gamma_{1}\right)=\{1,+,-\} \\
& \widetilde{\mathcal{A}}^{\star}\left(\Gamma_{0}\right)=\left\{\left(0,0, e_{1},(0)\right),(0,0, \mathbf{0},(1)),(0,1, \mathbf{0},(0)),(1,0, \mathbf{0},(0))\right\}, \quad \widetilde{\mathcal{A}}^{\star}\left(\Gamma_{1}\right)=\left\{\left(\mathbf{0}_{3}, \mathbf{0}_{3}, \mathbf{0}, \cdot\right)\right\}
\end{aligned}
$$

Figure 2: The connected trivalent 1-marked genus 1 graphs

In particular, $\mathrm{c}_{1 ; p, b}^{(d)}=0$ unless $d=0$ and $p=n-2+b$. Below we assume that the pair $(p, b)$ satisfies the last condition.

By (2.29), the contribution of an element $\left(d, p^{\prime}, b^{\prime}\right)$ of $\mathcal{S}_{\Gamma_{1}}(d, p, b)$ to $c_{1 ; p, b}^{(0)}$ is

$$
\begin{aligned}
\frac{(-1)^{b+b^{\prime}}}{2} \llbracket \Phi_{() ; \mathbf{0}}^{\left(0, \mathbf{0}_{3}\right)}(q) \frac{\Phi_{\widehat{p} ;-b}(q)}{\Phi_{0}(q)} \frac{\Phi_{p^{\prime} ;-b^{\prime}}(q)}{\Phi_{0}(q)} \frac{\Phi_{\widehat{p}^{\prime} ; 1+b^{\prime}}(q)}{\Phi_{0}(q)} \rrbracket_{q ; 0} \\
= \begin{cases}\frac{1}{2} \llbracket \Phi_{() ; \mathbf{0}}^{\left(0, \mathbf{0}_{3}\right)}(q) \frac{\Phi_{1 ; 0}(q)}{\Phi_{0}(q)} \frac{\Phi_{p^{\prime} ; 0}(q)}{\Phi_{0}(q)} \frac{\Phi_{\widehat{p}^{\prime} ; 1}(q)}{\Phi_{0}(q)} \rrbracket_{q ; 0}, & \text { if } b, b^{\prime}=0 ; \\
0, & \text { otherwise. }\end{cases}
\end{aligned}
$$

In particular, $\Gamma_{1}$ does not contribute to $c_{1 ; n-1,1}^{(0)}$. By (2.38), (2.39), and (2.15), it contributes

$$
\frac{1}{2} \llbracket n L(q) \frac{\Phi_{1}(q)}{\Phi_{0}(q)}-\frac{n^{2}-1}{12}\left(1-L(q)^{-n}\right) \|_{q ; 0}=0
$$

to $\mathrm{c}_{1 ; n-2,0}^{(0)}$.

The contribution of an element $\left(b^{\prime \prime}, \epsilon, \mathbf{c},(i)\right)$ of $\widetilde{\mathcal{A}}^{\star}\left(\Gamma_{0}\right)$ to $c_{1 ; p, b}^{(0)}$ is

$$
(-1)^{b} \llbracket \Phi_{(i) ; \mathbf{c}}^{(1,(\epsilon))}(q) \frac{\Phi_{\widehat{p} ; b^{\prime \prime}+\epsilon-b}(q)}{\Phi_{0}(q)} \rrbracket_{q ; 0}= \begin{cases}\llbracket \Phi_{(i) ; \mathbf{c}}^{(1,(\epsilon))}(q) \frac{\Phi_{1 ; b^{\prime \prime}+\epsilon}(q)}{\Phi_{0}(q)} \rrbracket_{q ; 0}, & \text { if } b=0 ; \\ -\llbracket \Phi_{0 ; \mathbf{0}}^{(1,(1))}(q) \rrbracket_{q ; 0}, & \text { if } b=1, \epsilon=1 \\ 0, & \text { otherwise. }\end{cases}
$$

Along with the conclusion of the previous paragraph, (2.38), and (2.15), this implies that

$$
\mathrm{c}_{1 ; n-1,1}^{(0)}=\frac{n}{24}, \quad \mathrm{c}_{1 ; n-2,0}^{(0)}=-\frac{(n-1) n}{48} .
$$

Along with (2.37), this establishes (2.36). These two coefficients can also be obtained directly from (2.35) and (2.21).

We now obtain (2.42) and the vanishing of the remaining coefficients $c_{1 ; p, b}^{(d)}$ in (2.37) from the recursion (2.33). The collections $\mathcal{P}_{1,1}^{(1)}$ and $\mathcal{P}_{1,1}^{(2)}$ contain one element each,

$$
\Gamma_{0} \equiv(1,(0,\{1\}, 1)) \quad \text { and } \quad \Gamma_{1} \equiv(0,((0,\{1\}, 1),(0, \emptyset, 2))) \text {, }
$$


respectively $\left(\mathcal{P}_{1,1}^{(2)}\right.$ also contains a copy of $\Gamma_{1}$ with the two triplets swapped); the collections $\mathcal{P}_{1,1}^{(m)}$ with $m \geq 3$ are empty. The elements of the collections $\mathcal{P}_{1,1}^{(m)}$ precisely correspond to the two 1-marked genus 1 graphs depicted in Figure 2 (this is because $N=1$; see Remark 2.4). The collections (2.30) corresponding to $\Gamma_{0}$ and $\Gamma_{1}$ are also as in the first computation, i.e.

$$
\widetilde{\mathcal{A}}_{1,(1)}^{\star}=\widetilde{\mathcal{A}}^{\star}\left(\Gamma_{0}\right) \quad \text { and } \quad \widetilde{\mathcal{A}}_{0,(1,2)}^{\star}=\widetilde{\mathcal{A}}^{\star}\left(\Gamma_{1}\right) .
$$

The associated collections (2.31) are given by

$$
\begin{gathered}
\mathcal{S}_{1,(1)}(d, t)=\left\{\left(p^{\prime}, b^{\prime}\right) \in \llbracket n \rrbracket \times \mathbb{Z}: p^{\prime}-b^{\prime}=2+n(d+t)\right\}, \\
\mathcal{S}_{0,(1,2)}(d, t)=\left\{\left(\mathbf{p}^{\prime}, \mathbf{b}^{\prime}\right) \in\left(\llbracket n \rrbracket \times \llbracket n \rrbracket^{2}\right) \times\left(\mathbb{Z} \times \mathbb{Z}^{2}\right):\left|\mathbf{p}^{\prime}\right|-\left|\mathbf{b}^{\prime}\right|=n+2+n(d+t)\right\},
\end{gathered}
$$

with $\left|\mathbf{b}^{\prime}\right|$ above denoting the sum of the three components of $\mathbf{b}^{\prime}$ (not of their absolute values).

We first determine the contribution of $\mathcal{P}_{1,1}^{(1)}$ in (2.33) with $(g, N)=(1,1)$. The first product in (2.33) vanishes unless

$$
d^{\prime}=d, \quad t^{\prime}=t, \quad b \geq 0, \quad b^{\prime}=-1-b, \quad p^{\prime}=n-1-p ;
$$

if these conditions hold, then this product equals $(-1)^{b}$. Along with the condition on $\left(p^{\prime}, b^{\prime}\right)$ in (2.43), this implies that $\mathcal{P}_{1,1}^{(1)}$ does not contribute to $c_{1 ; p, b}^{(d)} \equiv \mathrm{c}_{1 ; p, b}^{(d, 0)}$ unless $d=0$ and $p=n-2-b$. If these two conditions hold, then the contribution of each element of $\widetilde{\mathcal{A}}_{1,(1)}^{\star}=\widetilde{\mathcal{A}}^{\star}\left(\Gamma_{0}\right)$ is given by (2.41). The sums of these contributions over the four elements of $\widetilde{\mathcal{A}}_{1,(1)}^{\star}$ in the two possibly non-trivial cases are given by (2.42).

We next determine the contribution of $\mathcal{P}_{1,1}^{(2)}$ in (2.33) with $(g, N)=(1,1)$. We write triples $\mathbf{p}^{\prime}$ and $\mathbf{b}^{\prime}$ appearing in (2.44) as $\left(p^{\prime}, p_{+}, p_{-}\right)$and $\left(b^{\prime}, b_{+}, b_{-}\right)$, respectively. The first product in (2.33) vanishes unless (2.45) is satisfied and

$$
b_{+} \geq 0, \quad b_{-}=-1-b_{+}, \quad p_{-}=n-1-p_{+} ;
$$

if these conditions hold, then this product equals $(-1)^{b+b_{+}}$. Along with the condition on $\left(\mathbf{p}^{\prime}, \mathbf{b}^{\prime}\right)$ in (2.44), this implies that $\mathcal{P}_{1,1}^{(2)}$ does not contribute to $\mathrm{c}_{1 ; p, b}^{(d)} \equiv \mathrm{c}_{1 ; p, b}^{(d, 0)}$ unless $d=0$ and $p=n-2-b$. If these two conditions hold, then the contribution of $\widetilde{\mathcal{A}}_{1,(1,2)}^{\star}=\widetilde{\mathcal{A}}^{\star}\left(\Gamma_{1}\right)$ is half of (2.40) and thus still vanishes. This re-establishes (2.42) and the vanishing of the remaining coefficients $c_{1 ; p, b}^{(d)}$ in (2.37).

\subsection{Proofs of Theorems 1 and 2}

These two theorems for $g=0$ are established in 22. Theorem 2 is meaningless if $n=1$, while Theorem 2 in this case is justified immediately after its statement. Thus, we can assume that $n \geq 2$ and $2 g+N \geq 3$. We also assume that the numbers in (1.1) satisfy

$$
c_{s} \in\left\lfloor n \rrbracket \forall s \in[N] \quad \text { and } \quad \sum_{s=1}^{N}\left(b_{s}+c_{s}\right)=n d+(n-4)(1-g)+N .\right.
$$

By (2.1), the GW-invariant (1.1) is the coefficient of

$$
q^{d} \prod_{s=1}^{N} H^{\widehat{c}_{s}} \hbar_{s}^{-b_{s}-1}, \quad \text { where } \quad \widehat{c}_{s}=n-1-c_{s},
$$


on the right-hand side of (2.3).

For tuples $\mathbf{d} \equiv\left(d_{s}\right)_{s \in[N]}$ and $\mathbf{b}^{\prime} \equiv\left(b_{s}^{\prime}\right)_{s \in[N]}$ in $\left(\mathbb{Z}^{\geq 0}\right)^{N}$, define

$$
\mathbf{p}\left(\mathbf{d}, \mathbf{b}^{\prime}\right) \in \mathbb{Z}^{N} \quad \text { by } \quad p_{s}\left(\mathbf{d}, \mathbf{b}^{\prime}\right)=n d_{s}+\widehat{c}_{s}-b_{s}+b_{s}^{\prime} .
$$

By (2.3) and (2.2),

$$
\begin{aligned}
&\left\langle\tau_{b_{1}} H^{c_{1}}, \ldots, \tau_{b_{N}} H^{c_{N}}\right\rangle_{g, d}^{\mathbb{P}^{n-1}} \\
&=\sum_{d^{\prime}=0}^{d^{\prime}=d} \sum_{\substack{\mathbf{d} \in(\mathbb{Z} \geq 0)^{N} \\
|\mathbf{d}|=d-d^{\prime}}} \sum_{\substack{\mathbf{b}^{\prime} \in\left(\mathbf{Z}^{\prime} \geq 0\right)^{N} \\
\left|\mathbf{b}^{\prime}\right| \leq 3(g-1)+N}} \mathrm{c}_{g ; \mathbf{p}\left(\mathbf{d}, \mathbf{b}^{\prime}\right), \mathbf{b}^{\prime}}^{\left(d^{\prime}\right)} \prod_{s=1}^{s=N} \llbracket \llbracket F_{p_{s}\left(\mathbf{d}, \mathbf{b}^{\prime}\right)}(w, q) \rrbracket_{q ; d_{s}} \rrbracket_{w ; b_{s}-b_{s}^{\prime}},
\end{aligned}
$$

with $\llbracket \ldots \rrbracket_{q ; d}$ denoting the coefficient of $q^{d}$ and $\mathrm{c}_{g ; \mathbf{p}\left(\mathbf{d}, \mathbf{b}^{\prime}\right), \mathbf{b}^{\prime}}^{\left(d^{\prime}\right)} \equiv$ if $p_{s}\left(\mathbf{d}, \mathbf{b}^{\prime}\right) \notin \llbracket n \rrbracket$ for some $s \in[N]$.

For any non-vanishing summand on the right-hand side of (2.46),

$$
p_{s}(\mathbf{d}, \mathbf{b}) \leq n-1, \quad b_{s}+c_{s} \geq n d_{s} \quad \forall s \in[N] .
$$

Thus, $d_{s}=0$ if $b_{s}+c_{s}<n$. Since the coefficient of $q^{0}$ in $\widehat{F}_{p}(w, q)$ is 1 , it follows that $b_{s}^{\prime}=b_{s}$ and $p_{s}\left(\mathbf{d}, \mathbf{b}^{\prime}\right)=\widehat{c}_{s}$ in such a case. Since $\left|\mathbf{b}^{\prime}\right| \leq 3(g-1)+N$, this implies Theorem 2, By [22, Corollary 5.3],

$$
\left|\llbracket \llbracket F_{p_{s}\left(\mathbf{d}, \mathbf{b}^{\prime}\right)}(w, q) \rrbracket_{q ; d_{s}} \rrbracket_{w ; b_{s}-b_{s}^{\prime}}\right| \leq \frac{C_{n}^{d_{s}}}{\left(n d_{s}\right) !}
$$

for some $C_{n} \in \mathbb{R}^{+}$dependent only on $n$. Along with the next statement below, which is proved in the reminder of this section, (2.46) and (2.47) imply Theorem 1 .

For $\mathbf{b} \in\left(\mathbb{Z}^{\geq 0}\right)^{N}$ as above, let

$$
\mathbf{b} !=\prod_{s=1}^{s=N} b_{s}, \quad\left(\begin{array}{c}
|\mathbf{b}| \\
\mathbf{b}
\end{array}\right)=\left(\begin{array}{c}
|\mathbf{b}| \\
b_{1}, \ldots, b_{N}
\end{array}\right) .
$$

Proposition 2.5. Let $g, n \in \mathbb{Z}^{\geq 0}$ with $n \geq 2$. There exists $C_{n, g} \in \mathbb{R}$ such that

$$
\left|\mathrm{c}_{g ; \mathbf{p}, \mathbf{b}}^{(d)}\right| \leq \frac{N !}{\mathbf{b} !} C_{n, g}^{N+d} \quad \forall d \in \mathbb{Z}^{\geq 0}, \mathbf{p} \in \llbracket n \rrbracket^{N}, \mathbf{b} \in\left(\mathbb{Z}^{\geq 0}\right)^{N} .
$$

Lemma 2.6. Let $g \in \mathbb{Z}^{\geq 0}$. There exists $C_{g} \in \mathbb{R}$ such that

$$
\left|\llbracket \Phi_{I ; \mathbf{c}}^{(g, \underline{\epsilon})}(q) \rrbracket_{q ; d}\right| \leq \frac{(3(g-1)+m+|\mathbf{c}|) !}{|\mathbf{c}| ! \underline{\epsilon} !} C_{g}^{\|\mathbf{c}\|} \llbracket\left(1-C_{g} q\right)^{-\|\mathbf{c}\|-1} \rrbracket_{q ; d}\left(\begin{array}{c}
|\mathbf{c}| \\
\mathbf{c}
\end{array}\right) \prod_{r=1}^{\infty}\left(\frac{1}{r+1}\right)^{c_{r}}
$$

for all $I \in\left(\mathbb{Z}^{\geq 0}\right)^{g}, \mathbf{c} \in\left(\mathbb{Z}^{\geq 0}\right)^{\infty}, \underline{\epsilon} \in\left(\mathbb{Z}^{\geq 0}\right)^{m}$, and $m \in \mathbb{Z}^{\geq 0}$ with $2 g+m \geq 3$.

Proof. By (2.19) and (2.7), there exists $C_{g} \in \mathbb{R}$ such that

$$
\left|\widehat{A}_{I ; \mathbf{c}}^{(g, \underline{\epsilon})}\right| \leq \frac{(3(g-1)+m+|\mathbf{c}|) !}{\underline{\epsilon} !} C_{g} 2^{|\mathbf{c}|+\|\mathbf{c}\|}
$$


for all $I, \mathbf{c}, \underline{\epsilon}$, and $m$ as above. By (2.15), there exists $C_{n, g} \in \mathbb{R}$ such that

$$
\left|\llbracket \Phi_{0}(q)^{2-2 g} \rrbracket_{q ; d}\right|=\left|\llbracket(1+q)^{\frac{(n-1)(g-1)}{n}} \rrbracket_{q ; d}\right| \leq C_{n, g}^{d}=\llbracket\left(1-C_{n, g} q\right)^{-1} \rrbracket_{q ; d} .
$$

By [22, Lemma 5.6], there exists $C_{n} \in \mathbb{R}$ such that

$$
\left|\llbracket \frac{\Phi_{r}(q)}{\Phi_{0}(q)} \rrbracket_{q ; d}\right| \leq C_{n}^{r} \llbracket\left(1-C_{n} q\right)^{-r} \rrbracket_{q ; d} r ! \quad \forall r, d \in \mathbb{Z}^{\geq 0} .
$$

Combining (2.20) with the above three estimates, we obtain the claim.

For $g, N \in \mathbb{Z}^{\geq 0}$ with $2 g+N \geq 2$, let

$$
a_{g, N}=\sum_{\Gamma \in \mathcal{A}_{g, N+1}} \frac{1}{|\operatorname{Aut}(\Gamma)|} \prod_{v \in \operatorname{Ver}}\left(3(\mathfrak{g}(v)-1)+\left|\overline{\mathrm{Fl}}_{v}(\Gamma)\right|\right) !,
$$

with each $\Gamma$ as in the $S=[N+1]$ case of $(2.23)$.

Lemma 2.7. There exist $C \in \mathbb{R}^{+}$and $C_{g} \in \mathbb{R}$ for each $g \in \mathbb{Z}^{\geq 0}$ such that

$$
a_{g, N} \leq C_{g} C^{N} N ! \quad \forall g, N \in \mathbb{Z}^{\geq 0} \text { with } 2 g+N \geq 2 .
$$

Proof. We define $a_{0,0}=0, a_{0,1}=1$, and

$$
f_{g}(q)=\sum_{N=0}^{\infty} \frac{a_{g, N}}{N !} q^{N} \in \mathbb{Q}[[q]] \quad \forall g \in \mathbb{Z}^{\geq 0} .
$$

The claim of the lemma is equivalent to the existence of some $C \in \mathbb{R}^{+}$such that all power series $f_{g}(q)$ converge whenever $|q|<1 / C$. The $g=0$ case of this claim is [22, Lemma 5.10]. It implies that the power series

$$
f_{0}(q) \in q \mathbb{Q}[[q]], \quad\left(1+\ln \left(1-f_{0}(q)\right)^{-1},\left(1-f_{0}(q)\right)^{-m} \in \mathbb{Q}[[q]] \text { with } m \in \mathbb{Z}\right.
$$

converge for $|q|<1 / C$ for some $C \in \mathbb{R}^{+}$.

We thus assume that $g \in \mathbb{Z}^{+}$. Suppose $N \in \mathbb{Z}^{\geq 0}, \Gamma$ is a connected trivalent $(N+1)$-marked genus $g$ graph as in (2.23) with

$$
S=\llbracket N+1 \rrbracket \equiv\{0,1, \ldots, N\},
$$

and $v=\eta(0)$ is the vertex to which the marked point labeled by 0 is attached. Let $S_{v} \subset \llbracket N+1 \rrbracket$ be the subset of marked points attached to $v$, as in Section 2.3. Breaking $\Gamma$ at $v$ and replacing the flags at $v$ with marked points, we obtain connected strands similarly to Figure 4 on page 30. The set of these strands consists of

- a genus 0 two-vertex graph, with the marked point 0 at one of the vertices and another marked point at the other vertex;

- $m$ additional strands $\Gamma_{i}$, each of which is of genus $g_{i}$ and carries $N_{i} \in \mathbb{Z} \geq 0$ of the original marked points of the set $[N]$ and $\left(s_{i}+1\right) \in \mathbb{Z}^{+}$additional marked points so that $g_{i}+N_{i}+s_{i}>0$ and $\Gamma_{i}$ is a trivalent graph if $2 g_{i}+N_{i}+s_{i} \geq 2$. 
Since $\Gamma$ is a trivalent $N$-marked genus $g$ graph,

$$
2 \mathfrak{g}(v)+m+\sum_{i=1}^{i=m} s_{i} \geq 2, \quad \mathfrak{g}(v)+\sum_{i=1}^{i=m} g_{i}+\sum_{i=1}^{i=m} s_{i}=g, \quad \sum_{i=1}^{i=m} N_{i}=N .
$$

Summing over all possibilities for $\Gamma$, we thus obtain

$$
a_{g, N}=\sum_{g^{\prime}=0}^{g^{\prime}=g} \sum_{m=1}^{\infty} \sum_{\substack{\mathbf{g}, \mathbf{s} \in(\mathbb{Z} \geq 0)^{m} \\
2 g^{\prime}+m+|\mathbf{s}| \geq 2 \\
g^{\prime}+|\mathbf{g}|+|\mathbf{s}|=g}} \sum_{\substack{\mathbf{N} \in(\mathbb{Z} \geq 0)^{m} \\
|\mathbf{N}|=N}} \frac{\left(3 g^{\prime}+m+|\mathbf{s}|-2\right) !}{m !}\left(\begin{array}{c}
N \\
\mathbf{N}
\end{array}\right)^{i=m} \prod_{i=1}^{i=m} \frac{a_{g_{i}, N_{i}+s_{i}}}{\left(s_{i}+1\right) !}
$$

This is equivalent to

$$
f_{g}(q)=\sum_{g^{\prime}=0}^{g^{\prime}=g} \sum_{m=1}^{\infty} \sum_{\substack{\mathbf{g}, \mathbf{s} \in\left(\mathbb{Z} \geq 0 \\ 2 g^{\prime}+m+|\mathbf{s}| \geq 2 \\ g^{\prime}+|\mathbf{g}|+|\mathbf{s}|=g\right.}} \frac{\left(3 g^{\prime}+m+|\mathbf{s}|-2\right) !}{m !} \prod_{i=1}^{i=m}\left(\frac{1}{\left(s_{i}+1\right) !} \frac{\mathrm{d}^{s_{i}} f_{g_{i}}}{\mathrm{~d} q^{s_{i}}}(q)\right) .
$$

We note that

$$
3 g^{\prime}+\left|\left\{i \in[m]:\left(g_{i}, s_{i}\right) \neq(0,0)\right\}\right|+|\mathbf{s}| \geq 2
$$

above unless $\left(g_{i}, s_{i}\right)=(g, 0)$ for some $i \in[m]$ and $\left(g_{j}, s_{j}\right)=(0,0)$ for all $j \neq i$. Thus,

$$
\begin{aligned}
& f_{g}(q)=f_{g}(q) \sum_{m^{\prime}=1}^{\infty} \frac{f_{0}(q)^{m^{\prime}}}{m^{\prime}}+\sum_{g^{\prime}=0}^{g^{\prime}=g} \sum_{m=0}^{\infty} \sum_{\substack{\mathbf{g}, \mathbf{s} \in(\mathbb{Z} \geq 0)^{m} \\
2 g^{\prime}+m+|\mathbf{s}| \geq 2 \\
g^{\prime}+|\mathbf{g}|+|\mathbf{s}|=g \\
\left(g_{i}, s_{i}\right) \neq \mathbf{0} \forall i}}\left(\frac{\left(3 g^{\prime}+m+|\mathbf{s}|-2\right) !}{m !} \prod_{i=1}^{i=m}\left(\frac{1}{\left(s_{i}+1\right) !} \frac{\mathrm{d}^{s_{i}} f_{g_{i}}}{\mathrm{~d} q^{s_{i}}}(q)\right)\right. \\
& \left.\times \sum_{m^{\prime}=0}^{\infty}\left(\begin{array}{c}
3 g^{\prime}+m+m^{\prime}+|\mathbf{s}|-2 \\
m^{\prime}
\end{array}\right) f_{0}(q)^{m^{\prime}}\right) .
\end{aligned}
$$

Combining the above with the last identity in Lemma 5.1, we obtain

$$
\begin{aligned}
& \left(1+\ln \left(1-f_{0}(q)\right) f_{g}(q)\right. \\
& =\sum_{g^{\prime}=0}^{g^{\prime}=g} \sum_{m=0}^{\infty} \sum_{\substack{\mathbf{g}, \mathbf{s} \in(\mathbb{Z} \geq 0) m \\
2 g^{\prime}+m+|\mathbf{s}| \geq 2 \\
g^{\prime}+|\mathbf{g}|+|\mathbf{s}|=g \\
\left(g_{i}, s_{i}\right) \neq \mathbf{0} \forall i}} \frac{\left(3 g^{\prime}+m+|\mathbf{s}|-2\right) !}{m !\left(1-f_{0}(q)\right)^{3 g^{\prime}+m+|\mathbf{s}|-1}} \prod_{i=1}^{i=m}\left(\frac{1}{\left(s_{i}+1\right) !} \frac{\mathrm{d}^{s_{i}} f_{g_{i}}}{\mathrm{~d} q^{s_{i}}}(q)\right) .
\end{aligned}
$$

The right-hand side above is a finite sum (the terms with $m>g-g^{\prime}$ vanish) and $g_{i}<g$ for all $i \in[m]$ and for all summands in (2.50). Since the power series (2.49) converge for $|q|<1 / C$, it follows by induction that so does the power series $f_{g}(q)$. This establishes the claim.

Proof of Proposition 2.5. Let $a ! \equiv 0$ if $a<0$. We first note that

$$
\frac{\left(b^{\prime \prime}+\epsilon-b\right) !}{b^{\prime \prime} !} \leq \frac{\left(b^{\prime \prime}+\epsilon\right) !}{b^{\prime \prime} ! b !} \leq\left(\begin{array}{c}
b^{\prime \prime}+\epsilon \\
\epsilon
\end{array}\right) \frac{\epsilon !}{b !} \leq 2^{b^{\prime \prime}+\epsilon} \frac{\epsilon !}{b !} \quad \forall b, b^{\prime \prime}, \epsilon \in \mathbb{Z}^{\geq 0} .
$$


Furthermore,

$$
\begin{aligned}
\frac{\left(b_{-}^{\prime \prime}+\epsilon_{-}-b^{\prime}\right) !}{b_{-}^{\prime \prime} !} \frac{\left(b_{+}^{\prime \prime}+\epsilon_{+}+1+b^{\prime}\right) !}{b_{+}^{\prime \prime} !} & \leq\left(\begin{array}{c}
b_{-}^{\prime \prime}+b_{+}^{\prime \prime}+\epsilon_{-}+\epsilon_{+}+1 \\
b_{-}^{\prime \prime}, b_{+}^{\prime \prime}, \epsilon_{-}, \epsilon_{+}, 1
\end{array}\right) \epsilon_{-} ! \epsilon_{+} ! \\
& \leq 5^{b_{-}^{\prime \prime}+b_{+}^{\prime \prime}+\epsilon_{-}+\epsilon_{+}+1} \epsilon_{-} ! \epsilon_{+} !
\end{aligned}
$$

for all $b^{\prime}, b_{-}^{\prime \prime}, b_{+}^{\prime \prime}, \epsilon_{-}, \epsilon_{+} \in \mathbb{Z}^{\geq 0}$.

Let $\Gamma \in \mathcal{A}_{g, N}$ be as in (2.23). Since $\Gamma$ is a trivalent graph,

$$
2 g_{v}+\left|\overline{\mathrm{Fl}}_{v}(\Gamma)\right| \geq 3 \quad \forall v \in \operatorname{Ver}, \quad|\operatorname{Ver}| \leq 2\left(\sum_{v \in \operatorname{Ver}}\left(g_{v}-1\right)+|\mathrm{Edg}|\right)+N=2(g-1)+N ;
$$

the second statement above follows from the first one, the first statement in (2.25), and (2.24). Combining (2.24), the above bound, and the first statement in (2.25), we obtain

$$
|\operatorname{Edg}| \leq g-1+|\operatorname{Ver}| \leq 3(g-1)+N, \quad \sum_{v \in \operatorname{Ver}}\left(\left(g_{v}-1\right)+\left|\overline{\mathrm{Fl}}_{v}(\Gamma)\right|\right) \leq 4(g-1)+2 N .
$$

If in addition $\mathbf{b}^{\prime} \in\left(\mathbb{Z}^{\geq 0}\right)^{\operatorname{Edg}}$ and $v \in$ Ver, let

$$
\Delta_{v}\left(\mathbf{b}^{\prime}\right)=3\left(g_{v}-1\right)+3\left|\overline{\mathrm{Fl}}_{v}(\Gamma)\right|+1-\sum_{f \in \mathrm{Fl}_{v}^{-}(\Gamma)} b_{e_{f}}^{\prime}+\sum_{f \in \mathrm{Fl}_{v}^{+}(\Gamma)} b_{e_{f}}^{\prime} .
$$

By the above bounds,

$$
\sum_{v \in \operatorname{Ver}} \Delta_{v}\left(\mathbf{b}^{\prime}\right) \leq 14(g-1)+7 N
$$

Let $\left(\mathbf{b}^{\prime \prime}, \underline{\epsilon}, \mathbf{c}, \mathbf{I}\right) \in \widetilde{\mathcal{A}}^{\star}(\Gamma)$ and $\mathbf{b}^{\prime} \in\left(\mathbb{Z}^{\geq 0}\right)^{\mathrm{Edg}}$. By (2.26),

$$
\begin{aligned}
\left\|\mathbf{c}_{v}\right\|+1+\sum_{s \in S_{v}}\left(b_{s}^{\prime \prime}+\epsilon_{s}-b_{s}+1\right) & +\sum_{f \in \mathrm{Fl}_{v}^{-}(\Gamma)}\left(b_{f}^{\prime \prime}+\epsilon_{f}-b_{e_{f}}^{\prime}+1\right) \\
& +\sum_{f \in \mathrm{Fl}_{v}^{+}(\Gamma)}\left(b_{f}^{\prime \prime}+\epsilon_{f}+1+b_{e_{f}}^{\prime}+1\right) \leq \Delta_{v}\left(\mathbf{b}^{\prime}\right)
\end{aligned}
$$

for every $v \in$ Ver. Along with (2.51) and (2.52), this implies that

$$
\frac{1}{\epsilon_{v} !} \prod_{s \in S_{v}} \frac{\left(b_{s}^{\prime \prime}+\epsilon_{s}-b_{s}\right) !}{b_{s}^{\prime \prime} !} \prod_{f \in \mathrm{Fl}_{v}^{-}(\Gamma)} \frac{\left(b_{f}^{\prime \prime}+\epsilon_{f}-b_{e_{f}}^{\prime}\right) !}{b_{f}^{\prime \prime} !} \prod_{f \in \mathrm{Fl}_{v}^{+}(\Gamma)} \frac{\left(b_{f}^{\prime \prime}+\epsilon_{f}+1+b_{e_{f}}^{\prime}\right) !}{b_{f}^{\prime \prime} !} \leq 5^{\Delta_{v}\left(\mathbf{b}^{\prime}\right)} \prod_{s \in S_{v}} \frac{1}{b_{s} !} .
$$

By the first statement in [22, Corollary 5.8], there exists $C_{n} \in \mathbb{R}$ such that

$$
\left|\llbracket \frac{\Phi_{p ; b}(q)}{\Phi_{0}(q)} \rrbracket_{q ; d}\right| \leq C_{n}^{b} \llbracket\left(1-C_{n} q\right)^{-b-1} \rrbracket_{q ; d} b ! \quad \forall b, d \in \mathbb{Z}^{\geq 0}, p \in \llbracket n \Downarrow .
$$

By Lemma 2.6, (2.57), (2.55), and (2.56), there exists $C_{n, g} \in \mathbb{R}$ such that the absolute value of each nonzero factor $\llbracket \cdot \rrbracket$ in (2.29) is bounded above by

$$
\frac{\left(3\left(g_{v}-1\right)+\left|\overline{\mathrm{Fl}}_{v}(\Gamma)\right|+\left|\mathbf{c}_{v}\right|\right) !}{\left|\mathbf{c}_{v}\right| !}\left(\begin{array}{c}
\left|\mathbf{c}_{v}\right| \\
\mathbf{c}_{v}
\end{array}\right) \prod_{r=1}^{\infty}\left(\frac{1}{r+1}\right)^{c_{v ; r}} C_{n, g}^{\Delta_{v}\left(\mathbf{b}^{\prime}\right)} \llbracket\left(1-C_{n, g} q\right)^{-\Delta_{v}\left(\mathbf{b}^{\prime}\right)} \rrbracket_{q ; d_{v}} \prod_{s \in S_{v}} \frac{1}{b_{s} !}
$$


Along with (2.54), this implies that the absolute value of each summand in (2.29) is bounded above by

$$
\begin{aligned}
\frac{C_{n, g}^{14(g-1)+7 N} \llbracket\left(1-C_{n, g} q\right)^{-(14(g-1)+7 N)} \rrbracket_{q ; d}}{\mathbf{b !} !} & \times \prod_{v \in \operatorname{Ver}}\left(\frac{\left(3\left(g_{v}-1\right)+\left|\overline{\mathrm{Fl}}_{v}(\Gamma)\right|+\left|\mathbf{c}_{v}\right|\right) !}{\left|\mathbf{c}_{v}\right| !}\left(\begin{array}{c}
\left|\mathbf{c}_{v}\right| \\
\mathbf{c}_{v}
\end{array}\right) \prod_{r=1}^{\infty}\left(\frac{1}{r+1}\right)^{c_{v ; r}}\right) .
\end{aligned}
$$

By (2.26), the number of possibly nonzero factors in (2.29) with $\mathbf{c}_{v}$ fixed (and $\mathbf{b}^{\prime \prime}, \underline{\epsilon}$, and $I$ varying) is bounded above by

$$
\left(\begin{array}{c}
3\left(g_{v}-1\right)+\left|\overline{\mathrm{Fl}}_{v}(\Gamma)\right|-\left\|\mathbf{c}_{v}\right\|+g_{v}+2\left|\overline{\mathrm{Fl}}_{v}(\Gamma)\right|-1 \\
g_{v}+2\left|\overline{\mathrm{Fl}}_{v}(\Gamma)\right|-1
\end{array}\right) \leq 2^{4\left(g_{v}-1\right)+3\left|\overline{\mathrm{Fl}}_{v}(\Gamma)\right|-\left\|\mathbf{c}_{v}\right\|} .
$$

Along with the conclusion of the previous paragraph and (2.53), this implies that the absolute value of the sum in (2.29) with $\Gamma,\left(\mathbf{d}, \mathbf{p}^{\prime}, \mathbf{b}^{\prime}\right)$, and $\mathbf{c} \equiv\left(\mathbf{c}_{v}\right)_{v \in \text { Ver }}$ fixed is bounded above by

$$
\begin{aligned}
& \frac{C_{n, g}^{30(g-1)+15 N} \llbracket\left(1-C_{n, g} q\right)^{-(14(g-1)+7 N)} \rrbracket_{q ; d}}{\mathbf{b} !} \prod_{v \in \operatorname{Ver}}\left(3\left(g_{v}-1\right)+\left|\overline{\mathrm{Fl}}_{v}(\Gamma)\right|\right) ! \\
& \times 2^{-\|\mathbf{c}\|} \prod_{v \in \operatorname{Ver}}\left(\frac{\left(3\left(g_{v}-1\right)+\left|\overline{\mathrm{Fl}}_{v}(\Gamma)\right|+\left|\mathbf{c}_{v}\right|\right) !}{\left(3\left(g_{v}-1\right)+\left|\overline{\mathrm{Fl}}_{v}(\Gamma)\right|\right) !\left|\mathbf{c}_{v}\right| !}\left(\begin{array}{c}
\left|\mathbf{c}_{v}\right| \\
\mathbf{c}_{v}
\end{array}\right) \prod_{r=1}^{\infty}\left(\frac{1}{r+1}\right)^{c_{v ; r}}\right) .
\end{aligned}
$$

By Lemma 5.2, the sum of the terms on the second line above over all possibilities for $\mathbf{c}$ is bounded by $2^{3 g+N}$.

By (2.26) and the second equality in (2.25),

$$
\left|\mathbf{b}^{\prime}\right| \leq\left|\mathbf{b}^{\prime \prime}\right|+|\underline{\epsilon}| \leq 3(g-1)+N-|\operatorname{Edg}|
$$

for every nonzero summand $\llbracket \cdot \rrbracket$ in (2.29). Thus, the number of tuples $\mathbf{b}^{\prime}$ in (2.29) which contribute to (2.29) with $\Gamma$ and $\left(\mathbf{d}, \mathbf{p}^{\prime}\right)$ fixed is bounded above by

$$
\left(\begin{array}{c}
3(g-1)+N-|\operatorname{Edg}|+|\operatorname{Edg}|-1 \\
|\operatorname{Edg}|-1
\end{array}\right) \leq 2^{3(g-1)+N} .
$$

Along with (2.28) and the first statement in (2.53), this implies that the number of elements of $\mathcal{S}_{\Gamma}(d, \mathbf{p}, \mathbf{b})$ with nonzero summands in (2.29) is bounded above by

$$
2^{3(g-1)+N} \cdot n^{|\mathrm{Edg}|} \leq(2 n)^{3(g-1)+N} .
$$

By the conclusions of the last two paragraphs, there exists $C_{n, g} \in \mathbb{R}$ such that the absolute value of the contribution of each $\Gamma \in \mathcal{A}_{g, n}$ in (2.29) times $|\operatorname{Aut}(\Gamma)|$ is bounded above by

$$
\begin{aligned}
& \frac{C_{n, g}^{N}}{\mathbf{b} !}\left|\left(\begin{array}{c}
-(14(g-1)+7 N) \\
d
\end{array}\right)\right| C_{n, g}^{d} \cdot \prod_{v \in \operatorname{Ver}}\left(3\left(g_{v}-1\right)+\left|\overline{\mathrm{Fl}}_{v}(\Gamma)\right|\right) ! \\
& =\frac{C_{n, g}^{N}}{\mathbf{b} !}\left(\begin{array}{c}
14(g-1)+7 N+d-1 \\
d
\end{array}\right) C_{n, g}^{d} \cdot \prod_{v \in \operatorname{Ver}}\left(3\left(g_{v}-1\right)+\left|\overline{\mathrm{Fl}}_{v}(\Gamma)\right|\right) ! \\
& \leq \frac{C_{n, g}^{N}}{\mathbf{b} !} 2^{14(g-1)+7 N+d} C_{n, g}^{d} \cdot \prod_{v \in \text { Ver }}\left(3\left(g_{v}-1\right)+\left|\overline{\mathrm{Fl}}_{v}(\Gamma)\right|\right) ! ;
\end{aligned}
$$


on the first line above

$$
\left(\begin{array}{l}
a \\
b
\end{array}\right) \equiv \frac{a(a-1) \ldots(a-d+1)}{d !}
$$

is as in the Binomial Theorem. The claimed bound for $\mathrm{c}_{g ; \mathbf{p}, \mathbf{b}}^{(d)}$ now follows from Lemma 2.7.

\section{Torus equivariant setting}

In Section 3.1, we first review the relevant aspects of equivariant cohomology; a more detailed discussion can be found in [20, Section 1.1]. We then state an equivariant version of Theorem $\mathrm{A}$; see Theorem B. Theorem $\mathrm{A}$ is obtained from Theorem $\mathrm{B}$ by setting $\alpha=0$ and using the $l=0$ case of the second statement of [18, Theorem 5]. In Section 3.2, we apply the Virtual Equivariant Localization Theorem of [10] to reduce the generating series (3.8) for equivariant GW-invariant to a sum over the fixed loci of the actions of the $n$-torus $\mathbb{T}$ on the moduli spaces $\overline{\mathfrak{M}}_{g, N}\left(\mathbb{P}^{n-1}, d\right)$. The two proofs of Theorem B carried out in Sections 4.2 and 4.3 are outlined in Section 3.3 , they involve breaking the fixed loci into pieces of finitely many types for each fixed pair $(g, N)$. The technical observations and background data needed for these proofs are gathered in Section 3.4.

\subsection{Equivariant GW-invariants}

Denote by $\gamma \longrightarrow \mathbb{P}^{\infty}$ the tautological line bundle and by $\mathbb{T}$ the complex $n$-torus $\left(\mathbb{C}^{*}\right)^{n}$. Its group cohomology is the polynomial algebra on $n$ generators:

$$
H_{\mathbb{T}}^{*} \equiv H^{*}(B \mathbb{T} ; \mathbb{Q})=\mathbb{Q}[\underline{\alpha}] \equiv \mathbb{Q}\left[\alpha_{1}, \ldots, \alpha_{n}\right],
$$

where $\alpha_{i}=\pi_{i}^{*} c_{1}\left(\gamma^{*}\right)$ and

$$
\pi_{i}: B \mathbb{T} \equiv\left(\mathbb{P}^{\infty}\right)^{N} \longrightarrow B \mathbb{C}^{*} \equiv \mathbb{P}^{\infty}
$$

is the projection onto the $i$-th component. For $r \in \mathbb{Z}^{\geq 0}$, let $\sigma_{r} \in H_{\mathbb{T}}^{*}$ be the $r$-th elementary symmetric polynomial in $\alpha_{1}, \alpha_{2}, \ldots, \alpha_{n}$ and

$$
\widehat{\sigma}_{r}=(-1)^{r-1} \sigma_{r} \in H_{\mathbb{T}}^{*} .
$$

Denote by

$$
\mathcal{I} \subset \mathbb{Q}\left[\alpha_{1}, \ldots, \alpha_{n}\right]^{S_{n}} \subset H_{\mathbb{T}}^{*}
$$

the ideal generated by $\sigma_{1}, \sigma_{2}, \ldots, \sigma_{n-1}$ inside of the ring of symmetric polynomials.

If $\mathbb{T}$ is acting on a topological space $M$, let

$$
H_{\mathbb{T}}^{*}(M) \equiv H^{*}(B M ; \mathbb{Q}), \quad \text { where } \quad B M=E \mathbb{T} \times_{\mathbb{T}} M,
$$

be the equivariant cohomology of $M$. If the $\mathbb{T}$-action on $M$ lifts to an action on a (complex) vector bundle $V \longrightarrow M$, let

$$
\mathbf{e}(V) \equiv e(B V), \mathbf{c}(V) \equiv c(B V) \in H_{\mathbb{T}}^{*}(M)
$$

denote the equivariant Euler and Chern classes of $V$.

The projection map $B M \longrightarrow B \mathbb{T}$ induces an action of $H_{\mathbb{T}}^{*}$ on $H_{\mathbb{T}}^{*}(M)$. If in addition $M$ is a compact oriented manifold, this projection induces a well-defined integration-along-the-fiber homomorphism

$$
\int_{M}: H_{\mathbb{T}}^{*}(M) \longrightarrow H_{\mathbb{T}}^{*}
$$


for the fiber bundle $B M \longrightarrow B \mathbb{T}$. It commutes with the actions of $H_{\mathbb{T}}^{*}$. If $M^{\prime}$ is another compact oriented manifold with a $\mathbb{T}$-action, a $\mathbb{T}$-equivariant continuous map $f: M \longrightarrow M^{\prime}$ determines an equivariant cohomology push-forward homomorphism

$$
f_{*}: H_{\mathbb{T}}^{*}(M) \longrightarrow H_{\mathbb{T}}^{*}\left(M^{\prime}\right) .
$$

It is characterized by the property that

$$
\int_{M} \psi\left(f^{*} \psi^{\prime}\right)=\int_{M^{\prime}}\left(f_{*} \psi\right) \psi^{\prime} \in H_{\mathbb{T}}^{*} \quad \forall \psi \in H_{\mathbb{T}}^{*}(M), \psi^{\prime} \in H_{\mathbb{T}}^{*}\left(M^{\prime}\right) .
$$

The homomorphism (3.3) commutes with the actions of $H_{\mathbb{T}}^{*}$.

Throughout the paper we work with the standard action of $\mathbb{T}$ on $\mathbb{P}^{n-1}$ :

$$
\left(e^{\mathrm{i} \theta_{1}}, \ldots, e^{\mathrm{i} \theta_{n}}\right) \cdot\left[z_{1}, \ldots, z_{n}\right]=\left[e^{\mathrm{i} \theta_{1}} z_{1}, \ldots, e^{\mathrm{i} \theta_{n}} z_{n}\right] .
$$

It naturally lifts to the tautological line bundle $\gamma$ and to the tangent bundle $T \mathbb{P}^{n-1}$. Let

$$
\mathbf{x} \equiv \mathbf{e}\left(\gamma^{*}\right) \in H_{\mathbb{T}}^{2}\left(\mathbb{P}^{n-1}\right)
$$

be the equivariant hyperplane class. For $N \in \mathbb{Z}^{\geq 0}$, the $\mathbb{T}$-equivariant cohomology of $\mathbb{P}_{N}^{n-1}$ with respect to the induced diagonal $\mathbb{T}$-action on $\mathbb{P}_{N}^{n-1}$ is given by

$$
H_{\mathbb{T}}^{*}\left(\mathbb{P}_{N}^{n-1}\right)=\mathbb{Q}\left[\underline{\alpha}, \mathbf{x}_{1}, \ldots, \mathbf{x}_{n}\right] /\left\{\left(\mathbf{x}_{s}-\alpha_{1}\right) \ldots\left(\mathbf{x}_{s}-\alpha_{n}\right): s=1, \ldots, N\right\},
$$

where $\mathbf{x}_{s}=\pi_{s}^{*} \mathbf{x}$ and $\pi_{s}: \mathbb{P}_{N}^{n-1} \longrightarrow \mathbb{P}^{n-1}$ is the projection onto the $s$-th component. For each $\mathbf{p} \in\left\lfloor n \rrbracket^{N}\right.$, let

$$
\underline{\mathbf{x}}^{\mathbf{p}}=\prod_{s=1}^{s=N} \mathbf{x}_{s}^{p_{s}} \in H_{\mathbb{T}}^{*}\left(\mathbb{P}_{N}^{n-1}\right) ;
$$

these elements form a basis for $H_{\mathbb{T}}^{*}\left(\mathbb{P}_{N}^{n-1}\right)$ as a module over $H_{\mathbb{T}}^{*}=\mathbb{Q}[\underline{\alpha}]$.

For $g, N \in \mathbb{Z}^{\geq 0}$, the action of $\mathbb{T}$ on $\mathbb{P}^{n-1}$ induces an action on $\overline{\mathfrak{M}}_{g, N}\left(\mathbb{P}^{n-1}, d\right)$ so that the evaluation map

$$
\mathrm{ev}^{d} \equiv \mathrm{ev}_{1} \times \ldots \times \mathrm{ev}_{N}: \overline{\mathfrak{M}}_{g, N}\left(\mathbb{P}^{n-1}, d\right) \longrightarrow \mathbb{P}_{N}^{n-1}
$$

is $\mathbb{T}$-equivariant. By [2, 12], the moduli space $\overline{\mathfrak{M}}_{g, N}\left(\mathbb{P}^{n-1}, d\right)$ carries an equivariant virtual fundamental class. It defines a homomorphism (3.3) with $M=\overline{\mathfrak{M}}_{g, N}\left(\mathbb{P}^{n-1}, d\right)$ which satisfies (3.4) with $\int_{M}$ replaced by the integration against this class. In particular, there is a well-defined equivariant cohomology push-forward homomorphism

$$
\mathrm{ev}_{*}^{d} \equiv\left\{\operatorname{ev}_{1} \times \ldots \times \mathrm{ev}_{N}\right\}_{*}: H_{\mathbb{T}}^{*}\left(\overline{\mathfrak{M}}_{g, N}\left(\mathbb{P}^{n-1}, d\right)\right) \longrightarrow H_{\mathbb{T}}^{*}\left(\mathbb{P}_{N}^{n-1}\right) .
$$

It is characterized by the property that

$$
\int_{\left.\left[\overline{\mathfrak{M}}_{g, N}\left(\mathbb{P}^{n-1}, d\right)\right]\right]^{\mathrm{vir}}} \psi\left(\mathrm{ev}^{d *} \psi^{\prime}\right)=\int_{M^{\prime}}\left(\operatorname{ev}_{d *} \psi\right) \psi^{\prime} \in H_{\mathbb{T}}^{*}
$$

for all $\mathbb{T}$-equivariant cohomology classes $\psi$ on $\overline{\mathfrak{M}}_{g, N}\left(\mathbb{P}^{n-1}, d\right)$ and $\psi^{\prime}$ on $\mathbb{P}_{N}^{n-1}$. The homomorphism (3.6) commutes with the actions of $H_{\mathbb{T}}^{*}$. 
With $\mathrm{ev}_{*}^{d}$ as in (3.6), $\underline{\hbar}$ and $\underline{\hbar}^{-1}$ as in (2.1) , and $\underline{\mathbf{x}}=\left(\mathbf{x}_{1}, \ldots, \mathbf{x}_{n}\right)$, let

$$
\mathcal{Z}^{(g)}(\underline{\hbar}, \underline{\mathbf{x}}, q)=\sum_{d=0}^{\infty} q^{d} \operatorname{ev}_{*}^{d}\left\{\prod_{s=1}^{s=N} \frac{1}{\hbar_{s}-\psi_{s}}\right\} \in H_{\mathbb{T}}^{*}\left(\mathbb{P}_{N}^{n-1}\right)\left[\left[\underline{\hbar}^{-1}, q\right]\right] .
$$

For $g=0$ and $N=1,2$, we define the coefficient of $q^{0}$ in (3.8) to be

$$
1 \text { and } \quad-\frac{1}{\hbar_{1}+\hbar_{2}} \sum_{\substack{p_{1}, p_{2}, r \in \mathbb{Z} \geq 0 \\ p_{1}+p_{2}+r=n-1}} \widehat{\sigma}_{r} \mathbf{x}_{1}^{p_{1}} \mathbf{x}_{2}^{p_{2}},
$$

respectively. For each $p \in \llbracket n \rrbracket$, let

$$
\mathcal{Z}_{p}(\hbar, \mathbf{x}, q)=\mathbf{x}^{p}+\sum_{d=1}^{\infty} q^{d} \operatorname{ev}_{1 *}^{d}\left\{\frac{\operatorname{ev}_{2}^{d *} \mathbf{x}^{p}}{\hbar-\psi_{1}}\right\} \in H_{\mathbb{T}}^{*}\left(\mathbb{P}^{n-1}\right)\left[\left[\hbar^{-1}, q\right]\right],
$$

where $\mathrm{ev}_{1}^{d}, \mathrm{ev}_{2}^{d}: \overline{\mathfrak{M}}_{0,2}\left(\mathbb{P}^{n-1}, d\right) \longrightarrow \mathbb{P}^{n-1}$. For $\mathbf{p}=\left(p_{1}, p_{2}, \ldots, p_{N}\right) \in\left\lfloor n \rrbracket^{N}\right.$, define

$$
\mathcal{Z}_{\mathbf{p}}(\underline{\hbar}, \underline{\mathbf{x}}, q)=\prod_{s=1}^{s=N} \hbar_{s}^{-1} \mathcal{Z}_{p_{s}}\left(\hbar, \mathbf{x}_{s}, q\right)
$$

Theorem B. Suppose $n, N \in \mathbb{Z}^{+}$and $g \in \mathbb{Z}^{\geq 0}$ with $n \geq 2$ and $2 g+N \geq 3$. The generating function (3.8) for the equivariant $N$-pointed genus $g G W$-invariants of $\mathbb{P}^{n-1}$ is given by

$$
\mathcal{Z}^{(g)}(\underline{\hbar}, \underline{\mathbf{x}}, q)=\sum_{\mathbf{p} \in \llbracket n \rrbracket^{N}} \sum_{\mathbf{b} \in(\mathbb{Z} \geq 0)} \sum_{d=0}^{\infty} \mathcal{C}_{g ; \mathbf{p}, \mathbf{b}}^{(d)} q^{d} \underline{\hbar}^{-\mathbf{b}} \mathcal{Z}_{\mathbf{p}}(\underline{\hbar}, \underline{\mathbf{x}}, q)
$$

for some $\mathcal{C}_{g ; \mathbf{p}, \mathbf{b}}^{(d)} \in \mathbb{Q}[\alpha]$ such that

$$
\mathcal{C}_{g ; \mathbf{p}, \mathbf{b}}^{(d)}-\sum_{t=0}^{\infty} \mathrm{c}_{g ; \mathbf{p}, \mathbf{b}}^{(d, t)} \widehat{\sigma}_{n}^{t} \in \mathcal{I},
$$

where $\mathrm{c}_{g ; \mathbf{p}, \mathbf{b}}^{(d, t)} \in \mathbb{Q}$ are the numbers defined in Section 2.3.

The closed formula (4.15) and separately the recursion (4.26) compute the coefficients $\mathcal{C}_{g ; \mathbf{p}, \mathbf{b}}^{(d)}$ in (3.11) and thus provide a straightforward (though laborious) algorithm for computing the generating function (3.8) for the equivariant $N$-pointed genus $g \mathrm{GW}$-invariants of $\mathbb{P}^{n-1}$. Let

$$
\mathcal{Y}(\hbar, \mathbf{x}, q)=\sum_{d=0}^{\infty} \frac{q^{d}}{\prod_{r=1}^{r=d}\left(\prod_{k=1}^{k=n}\left(\mathbf{x}-\alpha_{k}+r \hbar\right)-\prod_{k=1}^{k=n}\left(\mathbf{x}-\alpha_{k}\right)\right)} \in\left(\mathbb{Q}_{\alpha ; \hbar, \mathbf{x}}^{\prime} \cap \mathbb{Q}_{\alpha}[\mathbf{x}]\left[\left[\hbar^{-1}\right]\right]\right)[[Q]]
$$

By [11, Section 29.1] and [22, Lemma A.1], the power series $\zeta(\mathbf{x}, q)$ and $\Psi_{b}(\mathbf{x}, q)$ in (3.31) are described by

$$
\mathcal{Y}(\hbar, \mathbf{x}, q)=e^{\zeta(\mathbf{x}, q) / \hbar} \sum_{b=0}^{\infty} \Psi_{b}(\mathbf{x}, q) \hbar^{b} .
$$

By the proofs of Lemma A.1 and Proposition 2.1 in [22], this relation determines $\zeta(\mathbf{x}, q)$ and $\Psi_{b}(\mathbf{x}, q)$ through an explicit recursion involving differential operators. By (3.39) and the proof 
of [22, Proposition 4.2], the power series $\Psi_{b}(\mathbf{x}, q)$ determine the power series $\Psi_{I ; \mathbf{c}}^{(g, \underline{\epsilon})}$ in (3.37) and $\Psi_{p ; b}(\mathbf{x}, q)$ in (3.33).

For concreteness, we now describe the power series $\zeta(\mathbf{x}, q)$ and $\Psi_{0}(\mathbf{x}, q)$ explicitly. For any $r \in \mathbb{Z}^{\geq 0}$ and any power series $f$, denote by $\sigma_{r}(f)$ the power series obtained from $f$ by taking the $r$-th elementary symmetric polynomial in $\left\{f-\alpha_{i}\right\}_{i \in[n]}$. Define

$$
L(\mathbf{x}, q) \in \mathbf{x}+q \cdot \mathbb{Q}\left[\alpha, \mathbf{x}, \sigma_{n-1}(\mathbf{x})^{-1}\right]\left[\left[\mathbf{x}^{-1} q\right]\right] \quad \text { by } \quad \sigma_{n}(L(\mathbf{x}, q))-q=\sigma_{n}(\mathbf{x}) .
$$

The power series $\zeta(\mathbf{x}, q)$ and $\Psi_{0}(\mathbf{x}, q)$ are described by

$$
\begin{gathered}
\zeta \in \mathbf{x} q \cdot \mathbb{Q}\left[\alpha, \mathbf{x}, \sigma_{n-1}(\mathbf{x})^{-1}\right][[q]], \quad \mathbf{x}+D \zeta(\mathbf{x}, q)=L(\mathbf{x}, q), \\
\Psi_{0}(\mathbf{x}, q)=\left(\frac{\mathbf{x} \sigma_{n-1}(\mathbf{x})}{L(\mathbf{x}, q) \sigma_{n-1}(L(\mathbf{x}, q))}\right)^{1 / 2}\left(\frac{L(\mathbf{x}, q)}{\mathbf{x}}\right)^{1 / 2}
\end{gathered}
$$

setting $\alpha=0$ and $\mathbf{x}=1$ above gives the first formulas in (2.14) and in (2.15).

As demonstrated in [6, 18, 20], equivariant localization computations in GW-theory can sometimes be carried out by working with the residues of the equivariant mirror B-side functions and by extracting the non-equivariant terms at the end. In such situations, precise knowledge of the equivariant coefficients $\mathcal{C}_{g ; \mathbf{p}, \mathbf{b}}^{(d)}$ in (3.11) is not avoidable.

\subsection{Equivariant localization setup}

Denote by

$$
\mathcal{H}_{\mathbb{T}}^{*}=\mathbb{Q}_{\alpha} \equiv \mathbb{Q}\left(\alpha_{1}, \ldots, \alpha_{n}\right)
$$

the field of fractions of $H_{\mathbb{T}}^{*}$. If $M$ is a topological space with a $\mathbb{T}$-action, let

$$
\mathcal{H}_{\mathbb{T}}^{*}(M)=H_{\mathbb{T}}^{*}(M) \otimes_{H_{\mathbb{T}}^{*}} \mathcal{H}_{\mathbb{T}}^{*} .
$$

In the case $M$ is a compact oriented manifold, the classical equivariant localization theorem of [1] relates the homomorphism (3.2) to the fixed locus of the $\mathbb{T}$-action. The latter is a union of compact orientable manifolds $F$ and $\mathbb{T}$ acts on the normal bundle $\mathcal{N} F$ of each $F$. Once an orientation of $F$ is chosen, there is a well-defined integration-along-the-fiber homomorphism

$$
\int_{F}: H_{\mathbb{T}}^{*}(F) \longrightarrow H_{\mathbb{T}}^{*}
$$

The localization theorem of [1] states that

$$
\int_{M} \psi=\sum_{F} \int_{F} \frac{\left.\psi\right|_{F}}{\mathbf{e}(\mathcal{N} F)} \in H_{\mathbb{T}}^{*} \subset \mathcal{H}_{\mathbb{T}}^{*} \quad \forall \psi \in H_{\mathbb{T}}^{*}(M),
$$

where the sum is taken over all components $F$ of the fixed locus of $\mathbb{T}$. Part of the statement of (3.13) is that $\mathbf{e}(\mathcal{N} F)$ is invertible in $\mathcal{H}_{\mathbb{T}}^{*}(F)$.

The standard action of $\mathbb{T}$ on $\mathbb{P}^{n-1}$ has $n$ fixed points:

$$
P_{1} \equiv[1,0, \ldots, 0], \quad P_{2} \equiv[0,1,0, \ldots, 0], \quad \ldots \quad P_{n} \equiv[0, \ldots, 0,1] .
$$


By the choice of the lift of $\mathbb{T}$-action to the tautological line bundle $\gamma$ over $\mathbb{P}^{n-1}$,

$$
\left.\mathbf{x}\right|_{P_{i}}=\alpha_{i} \in H_{\mathbb{T}}^{*}=H_{\mathbb{T}}^{*}\left(P_{i}\right) \quad \forall i \in[n] .
$$

Along with the $\mathbb{T}$-equivariance of Euler's sequence for $\mathbb{P}^{n-1}$, this implies that

$$
\left.\mathbf{e}\left(T \mathbb{P}^{n-1}\right)\right|_{P_{i}} \equiv \mathbf{e}\left(T_{P_{i}} \mathbb{P}^{n-1}\right)=\prod_{k \in[n]-i}\left(\alpha_{i}-\alpha_{k}\right) \in H_{\mathbb{T}}^{*}\left(P_{i}\right)=H_{\mathbb{T}}^{*}=\mathbb{Q}[\underline{\alpha}] \quad \forall i \in[n] .
$$

For each $i \in[n]$, define

$$
\phi_{i}=\prod_{k \neq i}\left(\mathbf{x}-\alpha_{k}\right) \in H_{\mathbb{T}}^{*}\left(\mathbb{P}^{n-1}\right) .
$$

This is the equivariant Poincare dual of $P_{i}$ in $\mathbb{P}^{n-1}$ in the sense of the $N=1$ case of (3.17) below.

The standard diagonal $\mathbb{T}$-action on $\mathbb{P}_{N}^{n-1}$ has $n^{N}$ fixed points:

$$
P_{i_{1} \ldots i_{N}} \equiv P_{i_{1}} \times \ldots \times P_{i_{N}}, \quad i_{1}, \ldots, i_{N} \in[n] .
$$

By (3.13) and (3.15), this implies that

$$
\left.\eta\right|_{P_{i_{1} \ldots i_{N}}} \equiv \int_{P_{i_{1} \ldots i_{N}}} \eta=\int_{\mathbb{P}_{N}^{n-1}} \eta \prod_{s=1}^{N} \pi_{i_{s}}^{*} \phi_{i_{s}} \in H_{\mathbb{T}}^{*} \quad \forall \eta \in H_{\mathbb{T}}^{*}\left(\mathbb{P}_{N}^{n-1}\right), i_{1}, \ldots, i_{N} \in[n] .
$$

Under the identifications (3.1) and (3.5), the restriction maps on the equivariant cohomology induced by the inclusions of $P_{i_{1} \ldots i_{N}}$ into $\mathbb{P}_{N}^{n-1}$ are the homomorphisms

$$
H_{\mathbb{T}}^{*}\left(\mathbb{P}_{N}^{n-1}\right) \longrightarrow H_{\mathbb{T}}^{*}, \quad \mathbf{x}_{s} \longrightarrow \alpha_{i_{s}}, s \in[N] .
$$

By (3.5) and (3.18), an element of $H_{\mathbb{T}}^{*}\left(\mathbb{P}_{N}^{n-1}\right)$ is determined by its restrictions

$$
\left.\eta\right|_{P_{i_{1} \ldots i_{N}}} \equiv \int_{P_{i_{1} \times \ldots \times P_{i_{N}}}} \eta \in H_{\mathbb{T}}^{*}
$$

to the $n^{N}$ fixed points of the $\mathbb{T}$-action on $\mathbb{P}_{N}^{n-1}$. Along with (3.17) and (3.7), this implies that the power series $\mathcal{Z}^{(g)}(\underline{\hbar}, \underline{\mathbf{x}}, q)$ in $(\underline{3.8})$ is determined by the $n^{N}$ power series

$$
\mathcal{Z}^{(g)}\left(\underline{\hbar}, \alpha_{i_{1}, \ldots, i_{N}}, q\right)=\sum_{d=0}^{\infty} q^{d} \int_{\overline{\mathfrak{M}}_{g, N}\left(\mathbb{P}^{n-1}, d\right)} \prod_{s=1}^{s=N} \frac{\mathrm{ev}_{s}^{*} \phi_{i_{s}}}{\hbar_{s}-\psi_{s}}
$$

where $\alpha_{i_{1} \ldots i_{N}} \equiv\left(\alpha_{i_{1}}, \ldots, \alpha_{i_{N}}\right)$.

The virtual localization theorem of [10] extends (3.13) to the integration against equivariant virtual fundamental classes. It in particular determines an induced $\mathbb{T}$-action on the virtual normal bundle $\mathcal{N} \mathcal{Z}_{\Gamma}^{\text {vir }}$ of each topological components $\mathcal{Z}_{\Gamma}$ of the fixed locus of the $\mathbb{T}$-action and reduces (3.19) to integrals over $\mathcal{Z}_{\Gamma}$. By [10, Section 4],

$$
\int_{\overline{\mathfrak{M}}_{g, N}\left(\mathbb{P}^{n-1}, d\right)} \prod_{s=1}^{s=N} \frac{\mathrm{ev}_{s}^{*} \phi_{i_{s}}}{\hbar_{s}-\psi_{s}}=\sum_{\Gamma \in \mathcal{A}_{g, N}(n, d)} \int_{\mathcal{Z}_{\Gamma}} \frac{1}{\mathbf{e}\left(\mathcal{N} \mathcal{Z}_{\Gamma}^{\mathrm{vir}}\right)} \prod_{s=1}^{s=N} \frac{\mathrm{ev}_{s}^{*} \phi_{i_{s}}}{\hbar_{s}-\psi_{s}}
$$


where $\mathcal{A}_{g, N}(n, d)$ is the set of equivalence classes of connected $[n]$-valued $N$-marked genus $g$ degree $d$ graphs; these are defined below. Part of the statement of (3.20) is that $\mathbf{e}\left(\mathcal{N} \mathcal{Z}_{\Gamma}^{\text {vir }}\right)$ is invertible in $\mathcal{H}_{\mathbb{T}}^{*}\left(\mathcal{Z}_{\Gamma}\right)$. In Section 4.2, we use (3.20) to first reduce (3.19) to a sum over the set $\mathcal{A}_{g, N}(n)$ of equivalence classes of connected $[n]$-valued $N$-marked genus $g$ graphs defined below. We then sum up over all possibilities for the $[n]$-values of the vertices to reduce the resulting sum to a sum over the collection $\mathcal{A}_{g, N}$ of connected trivalent $N$-marked genus $g$ graphs to obtain Theorem $\mathrm{B}$ with the first definition of the structure coefficients $c_{g ; \mathbf{p}, \mathbf{b}}^{(d)}$ in Section 2.3 .

An $[n]$-valued $S$-marked weighted graph is a tuple

$$
\Gamma \equiv\left((\mathfrak{g}, \mu): \operatorname{Ver} \longrightarrow \mathbb{Z}^{\geq 0} \times[n], \eta: S \sqcup \mathrm{Fl} \longrightarrow \operatorname{Ver}, \mathfrak{d}: \operatorname{Edg} \longrightarrow \mathbb{Z}^{+}\right)
$$

such that the tuple

$$
\Gamma_{0} \equiv\left(\mathfrak{g}: \operatorname{Ver} \longrightarrow \mathbb{Z}^{\geq 0}, \eta: S \sqcup \mathrm{Fl} \longrightarrow \operatorname{Ver}, \mathrm{Edg}\right)
$$

is an $S$-marked graph and

$$
\mu\left(f_{e}^{-}\right) \neq \mu\left(f_{e}^{+}\right) \quad \forall e \equiv\left\{f_{e}^{-}, f_{e}^{+}\right\} \in \operatorname{Edg} .
$$

The first diagram in Figure 3 represents an $[n]$-valued 2-marked weighted graph $\Gamma$ with $\mathfrak{g}(v)=0$ for all $v \in$ Ver. The values of $\mu$ on the vertices and of $\mathfrak{d}$ on the edges are indicated by the numbers next to the vertices and the edges. By (3.23), no two consecutive vertex labels are the same.

An equivalence between an $S$-marked weighted graph as in (3.21) and another $S$-marked weighted graph

$$
\Gamma^{\prime} \equiv\left(\left(\mathfrak{g}^{\prime}, \mu^{\prime}\right): \operatorname{Ver}^{\prime} \longrightarrow \mathbb{Z}^{\geq 0} \times[n], \eta^{\prime}: S \sqcup \mathrm{Fl}^{\prime} \longrightarrow \operatorname{Ver}^{\prime}, \mathfrak{d}^{\prime}: \operatorname{Edg}^{\prime} \longrightarrow \mathbb{Z}^{+}\right)
$$

is an equivalence $\left(h_{\mathrm{Ver}}, h_{\mathrm{Fl}}\right)$ between the associated $S$-marked graphs $\Gamma_{0}$ and $\Gamma_{0}^{\prime}$ such that

$$
\mu=\mu^{\prime} \circ h_{\mathrm{Ver}}, \quad \mathfrak{d}(e)=\mathfrak{d}^{\prime}\left(h_{\mathrm{Fl}}(e)\right) \quad \forall e \in \mathrm{Edg} .
$$

We denote by $\operatorname{Aut}(\Gamma)$ the group of automorphisms of $\Gamma$.

For $\Gamma$ as in (3.21), we denote by

$$
|\Gamma| \equiv \sum_{e \in \operatorname{Edg}} \mathfrak{d}(e)
$$

its degree. We call a vertex $v$ of $\Gamma$ trivalent if $v$ is a trivalent vertex of the associated $N$-marked graph $\Gamma_{0}$. We call $\Gamma$ connected if $\Gamma_{0}$ is connected. If $\Gamma$ is connected, we define its arithmetic genus $\mathfrak{a}(\Gamma)$ to be $\mathfrak{a}\left(\Gamma_{0}\right)$. Let $\mathcal{A}_{g, N}(n, d)$ be the set of (equivalence classes of) connected [ $\left.n\right]$-valued $N$-marked genus $g$ degree $d$ graphs and $\mathcal{A}_{g, N}(n, *)$ be the union of the sets $\mathcal{A}_{g, N}(n, d)$ over $d \in \mathbb{Z}$.

An $[n]$-valued $N$-marked graph is a tuple

$$
\Gamma \equiv\left((\mathfrak{g}, \mu): \text { Ver } \longrightarrow \mathbb{Z}^{\geq 0} \times[n], \eta:[N] \sqcup \mathrm{Fl} \longrightarrow \text { Ver, Edg }\right)
$$

such that the tuple (3.22) is an $N$-marked graph and $\mu$ satisfies (3.23). We define the notions of equivalence, trivalence, connectedness, and genus for such graphs via the associated graph (3.22) as above. We denote by $\operatorname{Aut}(\Gamma)$ the group of automorphisms of a graph $\Gamma$ as above and by $\mathcal{A}_{g, N}(n)$ the set of equivalence classes of connected trivalent $[n]$-valued $N$-marked genus $g$ graphs. 

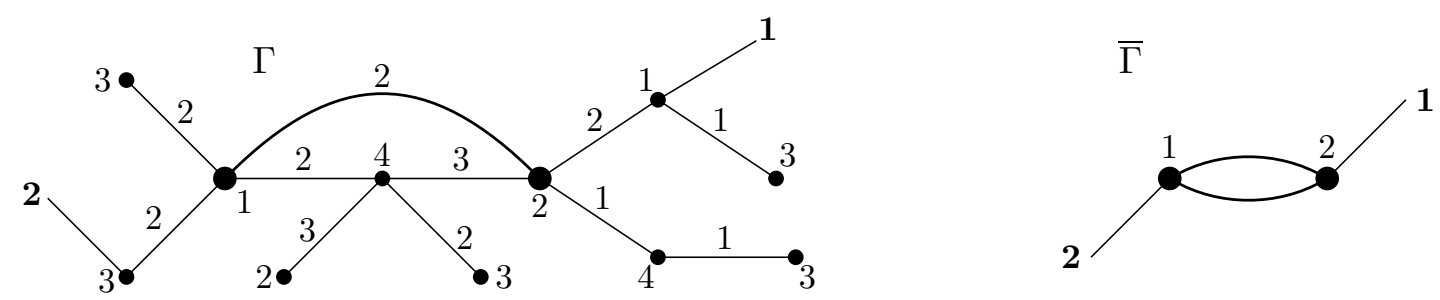

Figure 3: An $[n]$-valued 2-marked genus 1 degree 21 graph $\Gamma$, with special vertices indicated by larger dots, and its core $\bar{\Gamma}$.

\subsection{Outline of proofs of Theorem B}

Let $\Gamma$ be a connected $N$-marked genus $g$ graph as in $(2.23)$ with $2 \mathfrak{a}(\Gamma)+N \geq 3$. A vertex $v \in$ Ver such that $\operatorname{val}_{v}(\Gamma) \leq 0$ can then be contracted to obtain another connected $N$-marked genus $g$ graph

$$
\begin{gathered}
\Gamma^{\prime} \equiv\left(\mathfrak{g}^{\prime}: \operatorname{Ver}^{\prime} \longrightarrow \mathbb{Z}^{\geq 0}, \eta^{\prime}:[N] \sqcup \mathrm{Fl}^{\prime} \longrightarrow \operatorname{Ver}^{\prime}, \mathrm{Edg}^{\prime}\right) \quad \text { s.t. } \\
\operatorname{Ver}^{\prime}=\operatorname{Ver}-\{v\}, \quad \mathfrak{g}^{\prime}=\left.\mathfrak{g}\right|_{\mathrm{Ver}^{\prime}}, \quad \mathrm{Fl}^{\prime} \subset \mathrm{Fl} \cap \eta^{-1}\left(\operatorname{Ver}^{\prime}\right), \quad \eta^{\prime}=\eta \quad \text { on }\left([N] \cap \eta^{-1}\left(\operatorname{Ver}^{\prime}\right)\right) \sqcup \mathrm{Fl}^{\prime},
\end{gathered}
$$

as follows. If $\left|\mathrm{Fl} \cap \eta^{-1}(v)\right|=2$, we take

$$
\mathrm{Fl}^{\prime}=\mathrm{Fl} \cap \eta^{-1}\left(\operatorname{Ver}^{\prime}\right), \quad \operatorname{Edg}^{\prime}=\left\{e \in \operatorname{Edg}: e \cap \eta^{-1}(v)=\emptyset\right\} \sqcup\left\{\left\{f \in \mathrm{Fl}^{\prime}: e_{f} \cap \eta^{-1}(v) \neq \emptyset\right\}\right\} .
$$

If $\left|\mathrm{Fl} \cap \eta^{-1}(v)\right|=1$, we take

$$
\mathrm{Fl}^{\prime}=\left\{f \in \mathrm{Fl}: e_{f} \cap \eta^{-1}(v)=\emptyset\right\}, \quad \operatorname{Edg}^{\prime}=\left\{e \in \operatorname{Edg}: e \cap \eta^{-1}(v)=\emptyset\right\} .
$$

In this case, there is a unique $f^{c} \in \mathrm{Fl} \cap \eta^{-1}\left(\operatorname{Ver}^{\prime}\right)$ with $e_{f^{c}} \cap \eta^{-1}(v) \neq \emptyset$. We complete the definition of $\eta^{\prime}$ by requiring that

$$
\eta^{\prime}\left(S_{v}\right)=\left\{f^{c}\right\}
$$

the set $S_{v}$ consists of at most one element in this case (it is empty in the previous case).

Let $\Gamma$ be a connected $[n]$-valued $N$-marked genus $g$ weighted graph with $2 \mathfrak{a}(\Gamma)+N \geq 3$. We call the connected trivalent $[n]$-valued $N$-marked genus $g$ graph

$$
\bar{\Gamma} \equiv\left((\overline{\mathfrak{g}}, \bar{\mu}): \overline{\mathrm{Ver}} \longrightarrow \mathbb{Z}^{\geq 0} \times[n], \bar{\eta}:[N] \sqcup \overline{\mathrm{Fl}} \longrightarrow \overline{\mathrm{Ver}}, \overline{\mathrm{Edg}}\right)
$$

obtained by forgetting the map $\mathfrak{d}$ and repeatedly contracting the non-trivalent vertices of $\Gamma_{0}$ until all vertices become trivalent the core of $\Gamma$. It is independent of the choice of the order in which the non-trivalent vertices are contracted and satisfies

$$
\begin{gathered}
\{v \in \operatorname{Ver}: g(v) \geq 1\} \cup\{v \in \mathrm{Ver}:\{v\}=\eta(e) \text { for some } e \in \operatorname{Edg}\} \subset \overline{\mathrm{Ver}} \subset \mathrm{Ver}, \\
\overline{\operatorname{Edg}} \cap \operatorname{Edg}=\left\{e \in \operatorname{Edg}: \eta\left(e_{f}\right) \subset \overline{\mathrm{Ver}}\right\}, \quad\left\{f \in \mathrm{Fl}: e_{f} \in \overline{\operatorname{Edg}} \cap \operatorname{Edg}\right\} \subset \overline{\mathrm{Fl}} \subset \mathrm{Fl}, \\
(\overline{\mathfrak{g}}, \bar{\mu})=\left.(\mathfrak{g}, \mu)\right|_{\overline{\mathrm{Ver}}},\left.\quad \bar{\eta}\right|_{\left([N] \cap \eta^{-1}(\overline{\mathrm{Ver}})\right) \sqcup \overline{\mathrm{Fl}}}=\left.\eta\right|_{\left([N] \cap \eta^{-1}(\overline{\mathrm{Ver}})\right) \sqcup \overline{\mathrm{Fl}}} .
\end{gathered}
$$

We call the vertices $\overline{\mathrm{Ver}}$ of the core $\bar{\Gamma}$ the special vertices of $\Gamma$. The graph $\bar{\Gamma}$ on the right-hand side of Figure 3 is the core of the graph $\Gamma$ on its left-hand side. 

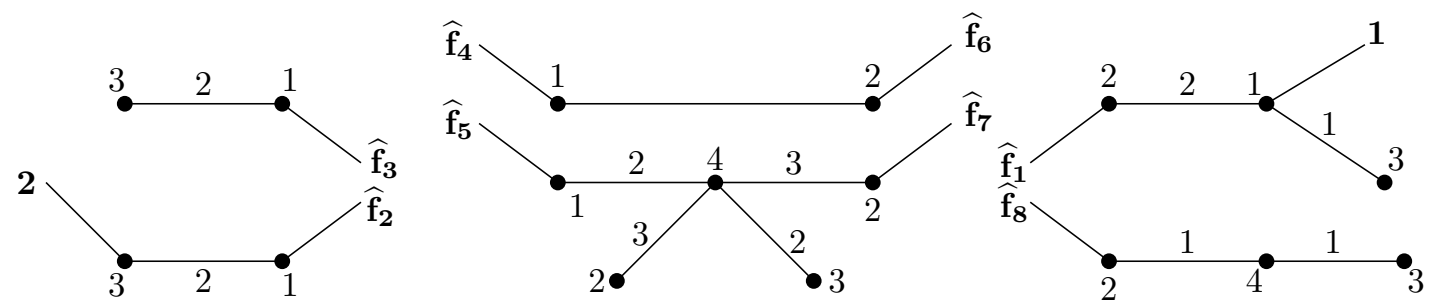

Figure 4: The strands of the graph in the first diagram in Figure 3 .

We compute (3.19) by breaking each graph $\Gamma \in \mathcal{A}_{g, N}(n, *)$ at its special vertices into strands

$$
\Gamma_{\mathfrak{p}} \equiv\left(\left(\mathfrak{g}_{\mathfrak{p}}, \mu_{\mathfrak{p}}\right): \operatorname{Ver}_{\mathfrak{p}} \longrightarrow \mathbb{Z}^{\geq 0} \times[n], \eta_{\mathfrak{p}}: S_{\mathfrak{p}} \sqcup \mathrm{Fl}_{\mathfrak{p}} \longrightarrow \operatorname{Ver}_{\mathfrak{p}}, \mathfrak{d}_{\mathfrak{p}}: \operatorname{Edg}_{\mathfrak{p}} \longrightarrow \mathbb{Z}^{+}\right)
$$

see Figure 4. The sets $\operatorname{Edg}_{\mathfrak{p}}$ of the edges of these strands partition the set Edg of the edges of $\Gamma$. Each edge $e \equiv\left\{f, f^{\prime}\right\}$ of $\Gamma$ so that $v \equiv \eta(f)$ is a special vertex of $\Gamma$ keeps a copy $v_{f}$ of $v$ with

$$
\mathfrak{g}_{\mathfrak{p}}\left(v_{f}\right)=0, \quad \mu_{\mathfrak{p}}\left(v_{f}\right)=\mu(v)
$$

We also add a marked point labeled by $\widehat{f}$ to this vertex. Thus, the collection $S_{\mathfrak{p}}$ of the marked points of the strands $\Gamma_{\mathfrak{p}}$ consists of the original $[N]$-marked points of $\Gamma$ and of a copy $\widehat{f}$ of each flag $f \in \mathrm{Fl}$ of $\Gamma$ so that $\mu(f) \in \overline{\mathrm{Ver}}$.

There are three types of strands $\Gamma_{\mathfrak{p}}$ :

(S1) genus 0 strands with one new marked point;

(S2) genus 0 strands with two new marked points;

(S3) genus 0 strands with one new marked point and one of the original $N$ marked points.

By (3.13), each one-pointed strand at a special vertex $v \in \overline{\operatorname{Ver}} \subset$ Ver contributes to

$$
\mathcal{Z}^{*}\left(\hbar, \alpha_{j}, q\right) \equiv \sum_{d=1}^{\infty} q^{d} \int_{\overline{\mathfrak{M}}_{0,1}\left(\mathbb{P}^{n-1}, d\right)} \frac{\mathrm{ev}_{1}^{*} \phi_{j}}{\hbar-\psi_{1}},
$$

where $j=\mu(v) \in[n]$ is the label of the vertex $v$ of $\Gamma$. By the dilaton relation [11, p527],

$$
\widetilde{\mathcal{Z}}^{*}\left(\hbar, \alpha_{j}, q\right) \equiv \sum_{d=1}^{\infty} q^{d} \int_{\overline{\mathfrak{M}}_{0,2}\left(\mathbb{P}^{n-1}, d\right)}\left(\frac{\mathrm{ev}_{1}^{*} \phi_{j}}{\hbar-\psi_{1}}\right)=\hbar^{-1} \mathcal{Z}^{*}\left(\hbar, \alpha_{j}, q\right) .
$$

Each of the two-pointed strands contributes to

$$
\mathcal{Z}^{*}\left(\hbar_{1}, \hbar_{2}, \alpha_{j_{1}}, \alpha_{j_{2}}, q\right) \equiv \sum_{d=1}^{\infty} q^{d} \int_{\overline{\mathfrak{M}}_{0,2}\left(\mathbb{P}^{n-1}, d\right)} \frac{\mathrm{ev}_{1}^{*} \phi_{j_{1}}}{\hbar_{1}-\psi_{1}} \frac{\mathrm{ev}_{2}^{*} \phi_{j_{2}}}{\hbar_{2}-\psi_{2}}
$$

where $j_{1}, j_{2} \in[n]$ are the labels of the vertices to which the marked points are attached. This implies that the power series $\mathcal{Z}^{(g)}(\underline{\hbar}, \underline{\mathbf{x}}, q)$ in (3.8) is determined by the previously computed power series for one- and two-pointed GW-invariants and by Hodge integrals over the Deligne-Mumford 
moduli spaces of stable curves.

While the number of one-marked strands at each node can be arbitrarily large, as indicated in [20, Sections 2.1,2.2] it is possible to sum over all possibilities for these strands at each special vertex; see Proposition 3.4 below. On the other hand, the numbers of special vertices, of two-pointed strands of type (S2), and of two-pointed strands of type (S3), are bounded (by $3 g+N)$. Using the Residue Theorem for $\mathbb{P}^{1}$, one can then sum up over all possibilities of the markings for each of the special nodes. Thus, the approach of breaking trees at special vertices reduces (3.19) to a finite sum, with one summand for each connected trivalent $N$-marked genus $g$ graph.

The first description of the structure constants $\mathrm{c}_{g ; \mathbf{p}, \mathbf{b}}^{(d)}$ after Theorem $\mathrm{A}$ is obtained by breaking every graph $\Gamma \in \mathcal{A}_{g, N}(n, d)$ at all special vertices of $\Gamma$. On the other hand, the second description is obtained by breaking each such graph at the special vertex $\bar{\eta}(N)$ only. In addition to the strands (S1), we then obtain strands $\Gamma_{\mathfrak{p}}$ of various genera $g_{\mathfrak{p}}$ with $N_{\mathfrak{p}} \in \mathbb{Z}^{+}$new marked points $\widehat{f}$ corresponding to the flags $f \in \mathrm{Fl}_{v}(\Gamma)$ with $e_{f} \in \operatorname{Edg}_{\mathfrak{p}}$ and subsets $S_{\mathfrak{p}}^{*} \subset[N]$ partitioning the original marked points $s$ of $\Gamma$ not lying on $v$ (i.e. $\eta(s) \neq v$ ). If $N \in S_{\mathfrak{p}}^{*}$ for some strand $\Gamma_{\mathfrak{p}}$, then $g_{\mathfrak{p}}=0, N_{\mathfrak{p}}=1$, and $S_{\mathfrak{p}}^{*}=\{N\}$. Setting $g_{s}=0, N_{s}=1$, and $S_{s}^{*}=\{s\}$ for $s \in S_{v}$, we thus obtain an element

$$
\left(g_{v},\left(g_{\mathfrak{p}}, S_{\mathfrak{p}}^{*}, N_{\mathfrak{p}}\right)_{\mathfrak{p} \in[m]}\right) \in \mathcal{P}_{g, N}^{(m)},
$$

where $m \in \mathbb{Z}^{+}$is the number the non-(S1) strands and of the marked points in $S_{v}$. With either approach, the main step is summing over all possibilities for the strands (S1), as is done in Proposition 3.4 .

\subsection{Key equivariant inputs}

With the notation as at the beginning of Section 3.1, let

$$
D_{\alpha}=\prod_{j \neq k}\left(\alpha_{j}-\alpha_{k}\right) \in \mathbb{Q}\left[\alpha_{1}, \ldots, \alpha_{n}\right]^{S_{n}} \subset H_{\mathbb{T}}^{*} .
$$

If $f=f(\hbar)$ is a rational function in $\hbar$ and $\hbar_{0} \in \mathbb{P}^{1}$, let

$$
\underset{\hbar=\hbar_{0}}{\mathfrak{R}}\{f(\hbar)\}=\frac{1}{2 \pi \mathfrak{i}} \oint f(\hbar) \mathrm{d} \hbar,
$$

where the integral is taken over a positively oriented loop around $\hbar=\hbar_{0}$ containing no other singular points of $f$, denote the residue of $f(\hbar) \mathrm{d} \hbar$ at $\hbar=\hbar_{0}$. With this definition,

$$
\underset{\hbar=\infty}{\mathfrak{R}}\{f(\hbar)\}=-\underset{w=0}{\mathfrak{R}}\left\{w^{-2} f\left(w^{-1}\right)\right\} .
$$

If $f$ involves variables other than $\hbar, \underset{\hbar=\hbar_{0}}{\Re}\{f(\hbar)\}$ will be a function of such variables. If $f$ is a power series in $q$ with coefficients that are rational functions in $\hbar$ and possibly other variables, denote by $\underset{\hbar=\hbar_{0}}{\mathfrak{R}}\{f(\hbar)\}$ the power series in $q$ obtained by replacing each of the coefficients by its residue at $\hbar=\hbar_{0}$. If $\hbar_{1}, \ldots, \hbar_{k}$ is a collection of distinct points in $\mathbb{P}^{1}$, let

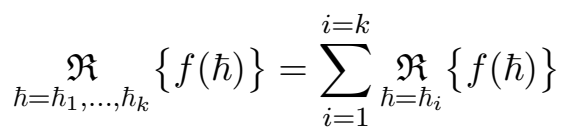


be the sum of the residues at the specified values of $\hbar$.

We denote by

$$
\mathbb{Q}_{\alpha}^{\prime} \equiv \mathbb{Q}\left[\alpha, \sigma_{n}^{-1}, D_{\alpha}^{-1}\right]^{S_{n}} \subset \mathbb{Q}_{\alpha}
$$

the subring of symmetric rational functions in $\alpha_{1}, \ldots, \alpha_{n}$ with denominators that are products of $\sigma_{n}$ and $D_{\alpha}$. Let

$$
\mathbb{Q}_{\alpha ; \hbar, \mathbf{x}}^{\prime} \equiv \mathbb{Q}_{\alpha}^{\prime}\left[\hbar, \mathbf{x}^{ \pm 1}\right]\left\langle(\mathbf{x}+r \hbar)^{n}-\mathbf{x}^{n}, \prod_{k=1}^{k=n}\left(\mathbf{x}-\alpha_{k}+r \hbar\right)-\prod_{k=1}^{k=n}\left(\mathbf{x}-\alpha_{k}\right) \mid r \in \mathbb{Z}^{+}\right\rangle \mathbb{Q}_{\alpha}(\hbar, \mathbf{x})
$$

be the subring of rational functions in $\alpha_{1}, \ldots, \alpha_{n}, \hbar$, and $\mathbf{x}$, symmetric in $\alpha_{1}, \ldots, \alpha_{n}$, with numerators that are polynomials in $\alpha_{1}, \ldots, \alpha_{n}, \hbar$, and $\mathbf{x}$, and with denominators that are products of

$$
\sigma_{n}, \quad D_{\alpha}, \quad \mathbf{x}, \quad(\mathbf{x}+r \hbar)^{n}-\mathbf{x}^{n}, \quad \prod_{k=1}^{k=n}\left(\mathbf{x}-\alpha_{k}+r \hbar\right)-\prod_{k=1}^{k=n}\left(\mathbf{x}-\alpha_{k}\right), \quad \text { with } r \in \mathbb{Z}^{+} .
$$

If $R$ is one of the rings $\mathbb{Q}_{\alpha}^{\prime}, \mathbb{Q}_{\alpha}^{\prime}\left[\mathbf{x}^{ \pm 1}\right]$, or $\mathbb{Q}_{\alpha ; \hbar, \mathbf{x}}^{\prime}$ and $f_{1}$ and $f_{2}$ are elements of $R$ or $R[[q]]$, we will write $f_{1} \sim f_{2}$ if $f_{1}-f_{2}$ lies in $\mathcal{I} \cdot R$ or $\mathcal{I} \cdot R[[q]]$, respectively. By the next lemma, certain operations on these rings respect these equivalence relations.

Lemma 3.1. (1) If $f \in \mathbb{Q}_{\alpha ; \hbar, \mathbf{x}}^{\prime}$, there exists $g \in \mathbb{Q}_{\alpha}^{\prime}\left[\mathbf{x}^{ \pm 1}\right]$ such that

$$
\underset{\hbar=0}{\Re}\left\{f\left(\hbar, \mathbf{x}=\alpha_{j}\right)\right\}=g\left(\mathbf{x}=\alpha_{j}\right) \quad \forall j \in[n] .
$$

(2) If $g \in \mathbb{Q}_{\alpha}^{\prime}\left[\mathbf{x}^{ \pm 1}\right]$,

$$
\underset{\mathbf{x}=0, \infty}{\mathfrak{R}}\left\{\frac{g(\mathbf{x})}{\prod_{k=1}^{k=n}\left(\mathbf{x}-\alpha_{k}\right)}\right\} \in \mathbb{Q}_{\alpha}^{\prime} .
$$

(3) For every $p \in \mathbb{Z}$,

$$
-\underset{\mathbf{x}=0, \infty}{\Re}\left\{\frac{\mathbf{x}^{p}}{\prod_{k=1}^{k=n}\left(\mathbf{x}-\alpha_{k}\right)}\right\} \sim \begin{cases}\widehat{\sigma}_{n}^{t}, & \text { if } p=n-1+n t \text { with } t \in \mathbb{Z} ; \\ 0, & \text { if } p+1 \notin n \mathbb{Z} .\end{cases}
$$

Proof. This is a modification of [22, Lemma 4.1], with the elements of $\mathcal{I}, \mathbb{Q}_{\alpha}^{\prime}$, and $\mathbb{Q}_{\alpha ; \hbar, \mathbf{x}}^{\prime}$ required to be symmetric in $\alpha_{1}, \ldots, \alpha_{n}$. The proof of [22, Lemma 4.1] applies verbatim in this setting.

We will also use the Residue Theorem on $\mathbb{P}^{1}$ :

$$
\sum_{\mathbf{x}_{0} \in \mathbb{P}^{1}} \underset{\mathbf{x}=\mathbf{x}_{0}}{\mathfrak{R}}\{f(\mathbf{x})\}=0
$$

for every rational function $f=f(\mathbf{x})$ on $\mathbb{P}^{1} \supset \mathbb{C}$. 
The most fundamental generating function for GW-invariants in the mirror symmetry computations following [8] is

$$
\begin{aligned}
\widetilde{\mathcal{Z}}(\hbar, \mathbf{x}, q) & \equiv 1+\widetilde{\mathcal{Z}}^{*}(\hbar, \mathbf{x}, q) \\
& \equiv 1+\sum_{d=1}^{\infty} q^{d} \operatorname{ev}_{1 *}^{d}\left\{\frac{1}{\hbar-\psi_{1}}\right\} \in H_{\mathbb{T}}^{*}\left(\mathbb{P}^{n-1}\right)\left[\left[\hbar^{-1}, q\right]\right],
\end{aligned}
$$

where $\operatorname{ev}_{1}^{d}: \overline{\mathfrak{M}}_{0,2}\left(\mathbb{P}^{n-1}, d\right) \longrightarrow \mathbb{P}^{n-1}$. By [8], $\widetilde{\mathcal{Z}}\left(\hbar, \alpha_{i}, q\right) \in \mathbb{Q}_{\alpha}(\hbar)$ for all $i \in[n]$. Thus, we can define

$$
\zeta\left(\alpha_{i}, q\right)=\underset{\hbar=0}{\mathfrak{R}}\left\{\ln \left(1+\widetilde{\mathcal{Z}}^{*}\left(\hbar, \alpha_{i}, q\right)\right)\right\} \in q \cdot \mathbb{Q}_{\alpha}[[q]]
$$

for each $i \in[n]$.

The proof of $\left[22\right.$, Proposition 4.2] provides power series $\Psi_{0}, \Psi_{1}, \ldots \in \mathbb{Q}_{\alpha}^{\prime}\left[\mathbf{x}^{ \pm 1}\right][[q]]$ such that

$$
\begin{gathered}
\Psi_{0}(0)=1, \quad \Psi_{b}(0)=0 \quad \forall b \in \mathbb{Z}^{+}, \quad \Psi_{b}(\mathbf{x}, q) \sim \Phi_{b}\left(q / \mathbf{x}^{n}\right) \mathbf{x}^{-b} \quad \forall b \in \mathbb{Z}^{\geq 0}, \\
\widetilde{\mathcal{Z}}\left(\hbar, \alpha_{i}, q\right)=e^{\zeta\left(\alpha_{i}, q\right) / \hbar} \sum_{b=0}^{\infty} \Psi_{b}\left(\alpha_{i}, q\right) \hbar^{b} \quad \forall i \in[n] .
\end{gathered}
$$

Furthermore, for every $p \in\left\lfloor n \rrbracket\right.$ there exist $\Psi_{b ; 0}, \Psi_{b ; 1}, \ldots \in \mathbb{Q}_{\alpha}^{\prime}\left[\mathbf{x}^{ \pm 1}\right][[q]]$ such that

$$
\begin{aligned}
\Psi_{p ; b}(\mathbf{x}, q) & \sim \Phi_{p ; b}\left(q / \mathbf{x}^{n}\right) \mathbf{x}^{p-b} & & \forall b \in \mathbb{Z}^{\geq 0}, \\
\mathcal{Z}_{p}\left(\hbar, \alpha_{i}, q\right) & =e^{\zeta\left(\alpha_{i}, q\right) / \hbar} \sum_{b=0}^{\infty} \Psi_{p ; b}\left(\alpha_{i}, q\right) \hbar^{b} & & \forall i \in[n] .
\end{aligned}
$$

Lemma 3.2. There exists a collection $\left\{\mathcal{C}_{p_{-} p_{+}}\right\}_{p_{ \pm} \in \llbracket n \rrbracket} \subset \mathbb{Q}[\underline{\alpha}]^{S_{n}}[[q]]$ such that

$$
\begin{aligned}
\underset{\hbar_{-}=0}{\mathfrak{R}}\left\{\frac{e^{-\frac{\zeta\left(\alpha_{i_{-}}, q\right)}{\hbar_{-}}}}{\hbar_{-}^{1+b_{-}}} \mathcal{Z}\left(\hbar_{-}, \hbar_{+}, \alpha_{i_{-}}, \alpha_{i_{+}}, q\right)\right\} \\
\quad=\sum_{b=0}^{b=b_{-}}\left(\frac{(-1)^{b}}{\hbar_{+}^{b+1}} \sum_{p_{-}, p_{+} \in \llbracket n \rrbracket} \mathcal{C}_{p_{-} p_{+}}(q) \Psi_{p_{-} ; b_{-}-b}\left(\alpha_{i_{-}}, q\right) \mathcal{Z}_{p_{+}}\left(\hbar_{+}, \alpha_{i_{+}}, q\right)\right)
\end{aligned}
$$

for all $b_{-} \in \mathbb{Z}^{\geq 0}$ and $i_{-}, i_{+} \in[n]$ and

$$
\mathcal{C}_{p_{-} p_{+}}(q) \sim \begin{cases}1, & \text { if } p_{-}+p_{+}=n-1 \\ 0, & \text { otherwise. }\end{cases}
$$

Proof. The proof of [22, Lemma 4.4] establishes the present lemma (the equivalence $\sim$ in the statement of $[22$, Lemma 4.4] is taken with respect to the ideal $\mathcal{I}$ inside of the entire ring $\mathbb{Q}[\alpha]$ ).

Corollary 3.3. For all $b_{-}, b_{+} \in \mathbb{Z}^{\geq 0}$ and $i_{-}, i_{+} \in[n]$,

$$
\begin{aligned}
\underset{\hbar_{+}=0}{\mathfrak{R}}\left\{\underset{\hbar_{-}=0}{\mathfrak{R}}\left\{\frac{e^{-\frac{\zeta\left(\alpha_{i_{-}}, q\right)}{\hbar_{-}}-\frac{\zeta\left(\alpha_{i_{+}}, q\right)}{\hbar_{+}}}}{\hbar_{-}^{1+b_{-}} \hbar_{+}^{1+b_{+}}} \mathcal{Z}\left(\hbar_{-}, \hbar_{+}, \alpha_{i_{-}}, \alpha_{i_{+}}, q\right)\right\}\right\} \\
=\sum_{b^{\prime}=0}^{b^{\prime}=b_{-}} \sum_{p_{-}, p_{+} \in \llbracket n \rrbracket}(-1)^{b^{\prime}} \mathcal{C}_{p_{-} p_{+}}(q) \Psi_{p_{-} ; b_{-}-b^{\prime}}\left(\alpha_{i_{-}}, q\right) \Psi_{p_{+} ; b_{+}+1+b^{\prime}}\left(\alpha_{i_{+}}, q\right) .
\end{aligned}
$$


Proof. This follows immediately from Lemma 3.2 and (3.33).

Let $g \in(\mathbb{Z})^{\geq 0}$. By the $\mathbb{T}$-equivariance of Euler's sequence for $\mathbb{P}^{n-1}$ and (2.18), there exist $h_{g, n ; I} \in \mathcal{I}[\mathbf{x}]$ with $I \in(\mathbb{Z} \geq 0)^{g}$ such that

$$
\begin{aligned}
\mathbf{e}\left(\mathbb{E}_{g}^{*} \otimes T_{P_{i}} \mathbb{P}^{n-1}\right)= & \left.\left(\sum_{I \in(\mathbb{Z} \geq 0)^{g}}\left(C_{g, n ; I} \mathbf{x}^{(n-1) g-\|I\|}+h_{g, n ; I}(\mathbf{x})\right) \lambda_{g ; I}\right)\right|_{\mathbf{x}=\alpha_{i}} \\
& \in H_{\mathbb{T}}^{*}\left(\overline{\mathcal{M}}_{g ; m} \times P_{i}\right)=H^{*}\left(\overline{\mathcal{M}}_{g ; m}\right)[\underline{\alpha}]
\end{aligned}
$$

for all $m \in \mathbb{Z}^{\geq 0}$ with $2 g+m \geq 0$ and $i \in[n]$. For $m \in \mathbb{Z}^{\geq 0}$ and $k \in[m]$, we denote the $k$-th component of $\mathbf{b} \in\left(\mathbb{Z}^{\geq 0}\right)^{m}$ by $b_{k} \in \mathbb{Z}^{\geq 0}$. If $m \in \mathbb{Z}^{\geq 0}$ with $2 g+m \geq 0, I \in\left(\mathbb{Z}^{\geq 0}\right)^{g}, \mathbf{b} \in\left(\mathbb{Z}^{\geq 0}\right)^{m}$, and $i \in[n]$, let

$$
\begin{aligned}
& \widetilde{\mathcal{Z}}_{\mathbf{b}, I}^{(g)}\left(\alpha_{i}, q\right)=\left(C_{g, n ; I} \alpha_{i}^{(n-1) g-\|I\|}+h_{g ; n ; I}\left(\alpha_{i}\right)\right) \\
& \times \sum_{m^{\prime}=0}^{\infty} \sum_{\substack{\mathbf{b}^{\prime} \in(\mathbb{Z} \geq 0) m^{\prime} \\
|\mathbf{b}|+\left|\mathbf{b}^{\prime}\right|=\mu_{g}(I)+m+m^{\prime}}}\left(\frac{\left\langle\left\langle\lambda_{g ; I} ; \widetilde{\tau}_{\mathbf{b}^{\prime}}\right\rangle\right\rangle}{m^{\prime} !} \prod_{k \in\left[m^{\prime}\right]} \underset{\substack{\hbar=0 \\
k}}{\Re}\left\{\frac{(-\hbar)^{-b_{k}^{\prime}}}{b_{k}^{\prime} !} \widetilde{\mathcal{Z}}^{*}\left(\hbar, \alpha_{i}, q\right)\right\}\right) .
\end{aligned}
$$

Each residue above is an element of $\mathbb{Q}_{\alpha}[[q]]$. Since the power series $\widetilde{\mathcal{Z}}^{*}(\hbar, \mathbf{x}, q)$ has no $q$-constant term, the above sum is finite in each $q$-degree. By Section 4.3 , the power series $\widetilde{\mathcal{Z}}_{\mathbf{b}, I}^{\left(g^{\prime}\right)}\left(\alpha_{i}, q\right)$ describe the contributions to (3.19) of the strands (S1) of the graphs $\Gamma$ with a fixed core $\bar{\Gamma}$ at a vertex $v$ of $\bar{\Gamma}$ with $\left|\overline{\mathrm{Fl}}_{v}(\bar{\Gamma})\right|=m$ and $\bar{\mu}(v)=i$.

Proposition 3.4. Let $g, m \in \mathbb{Z}^{\geq 0}$ with $2 g+m \geq 3$ and $I \in\left(\mathbb{Z}^{\geq 0}\right)^{g}$. There exist $\Psi_{I ; \mathbf{c}}^{(g, \epsilon)} \in \mathbb{Q}_{\alpha}^{\prime}\left[\mathbf{x}^{ \pm 1}\right][[q]]$ with $\mathbf{c} \in\left(\mathbb{Z}^{\geq 0}\right)^{\infty}$ and $\underline{\epsilon} \in\left(\mathbb{Z}^{\geq 0}\right)^{m}$ such that

$$
\begin{aligned}
\widetilde{\mathcal{Z}}_{\mathbf{b}, I}^{(g)}\left(\alpha_{i}, q\right)=\sum_{\mathbf{c} \in(\mathbb{Z} \geq 0)^{\infty}} & \sum_{\substack{\epsilon \in(\mathbb{Z} \geq 0) m \\
|\underline{\epsilon}| \leq \mu_{g}(I)+m \\
\epsilon_{k} \leq b_{k} \forall k \in[m]}}\left((-1)^{\mu_{g}(I)+m-\|\mathbf{c}\|} \Psi_{I ; \mathbf{c}}^{(g, \underline{c})}\left(\alpha_{i}, q\right)\right. \\
& \left.\quad \times \zeta\left(\alpha_{i}, q\right)^{|\mathbf{b}|-\left(\mu_{g}(I)+m-\|\mathbf{c}\|\right)}\left(\begin{array}{c}
|\mathbf{b}|-|\underline{\epsilon}| \\
\mu_{g}(I)+m-\|\mathbf{c}\|-|\underline{\epsilon}|
\end{array}\right) \prod_{k=1}^{m} \frac{b_{k} !}{\left(b_{k}-\epsilon_{k}\right) !}\right)
\end{aligned}
$$

for all $\mathbf{b} \in\left(\mathbb{Z}^{\geq 0}\right)^{m}$ and $i \in[n]$ and

$$
\Psi_{I ; \mathbf{c}}^{(g, \underline{\epsilon})}(\mathbf{x}, q) \sim \frac{\Phi_{I ; \mathbf{c}}^{(g, \underline{\epsilon})}\left(q / \mathbf{x}^{n}\right)}{\Phi_{0}\left(q / \mathbf{x}^{n}\right)^{m}} \mathbf{x}^{(n-1) g-\|I\|-\|\mathbf{c}\|} .
$$

Proof. By the first two statements in (3.30), (3.31), and Proposition 5.8, (3.37) holds with

$$
\begin{aligned}
\Psi_{I ; \mathbf{c}}^{(g, \underline{\epsilon})}(\mathbf{x}, q)=\frac{(-1)^{\mu_{g}(I)+m+|\mathbf{c}|}\left(C_{g, n ; I} \mathbf{x}^{(n-1) g-\|I\|}+h_{g ; n ; I}(\mathbf{x})\right) \widehat{A}_{I ; \mathbf{c}}^{(g, \underline{\mathbf{c}})}}{\Psi_{0}(\mathbf{x}, q)^{2 g-2+m}} \\
\times \prod_{r=1}^{\infty} \frac{1}{c_{r} !}\left(\frac{\Psi_{r}(\mathbf{x}, q)}{(r+1) ! \Psi_{0}(\mathbf{x}, q)}\right)^{c_{r}} .
\end{aligned}
$$

By the last statement in (3.30) and (2.20), $\Psi_{I ; \mathbf{c}}^{(g, \epsilon)}$ satisfies (3.38). 


\section{Proof of Theorem B}

We prove Theorem $\mathrm{B}$, with each of the two definitions of the structure constants $\mathrm{c}_{g ; \mathbf{p}, \mathbf{b}}^{(d)}$, by summing up the contributions of the $\mathbb{T}$-fixed loci $\mathcal{Z}_{\Gamma}$ of $\overline{\mathfrak{M}}_{g, N}\left(\mathbb{P}^{n-1}, d\right)$ as in (3.20) over all possibilities for the graph $\Gamma$ as in (3.21). As outlined in Section 3.3, this will be done by breaking each $\Gamma$ (and correspondingly each fixed locus $\mathcal{Z}_{\Gamma}$ ) either at every special vertex of $\Gamma$ or only at the special vertex $v=\bar{\mu}(N)$.

\subsection{Some preparation and notation}

Sections 4.2 and 4.3 describe coefficients $\mathcal{C}_{g ; \mathbf{p}, \mathbf{b}}^{(d)} \in \mathbb{Q}_{\alpha}$ by a closed formula and via a recursion, respectively, so that (3.11) is satisfied and (3.12) holds with $t \in \mathbb{Z}$ instead of $\mathbb{Z} \geq 0$. These coefficients are symmetric in $\alpha_{1}, \ldots, \alpha_{n}$ (this is also implied by the proof of the first claim below). The full statement of Theorem $\mathrm{B}$ then follows from the next observation.

Lemma 4.1. Let $g, n, N \in \mathbb{Z}^{\geq 0}$ be as in Theorems $\triangle$ and $\mathbb{B}$. If $\mathcal{C}_{g ; \mathbf{p}, \mathbf{b}}^{(d)} \in \mathbb{Q}_{\alpha}$ are such that (3.11) is satisfied, then $\mathcal{C}_{g ; \mathbf{p}, \mathbf{b}}^{(d)} \in \mathbb{Q}[\alpha]$. If in addition (3.12) holds with $t \in \mathbb{Z}$ instead of $\mathbb{Z} \geq 0$ for some $\mathrm{c}_{g ; \mathbf{p}, \mathbf{b}}^{(d, t)} \in \mathbb{Q}$, then it holds as stated with same coefficients $\mathrm{c}_{g ; \mathbf{p}, \mathbf{b}}^{(d, t)}$.

Proof. Let $\mathbf{b} \in\left(\mathbb{Z}^{\geq 0}\right)^{N}$ and $d \in \mathbb{Z}^{\geq 0}$. By (3.9) -( (3.11) , the coefficient of

$$
q^{d} \prod_{s=1}^{s=N}\left(\left(\hbar_{s}^{-1}\right)^{b_{s}+1}\right)
$$

in the power series $\mathcal{Z}^{(g)}(\underline{\hbar}, \underline{\mathbf{x}}, q)$ is

$$
\begin{aligned}
& \llbracket \mathcal{Z}^{(g)}(\underline{\hbar}, \underline{\mathbf{x}}, q) \|_{\underline{\hbar}^{-1}, q ; \mathbf{b}+\mathbf{1}, d}=\sum_{\mathbf{p} \in \llbracket n \rrbracket^{N}} \mathcal{C}_{g ; \mathbf{p}, \mathbf{b}}^{(d)} \underline{\mathbf{x}}^{\mathbf{p}} \\
& \quad+\sum_{\substack { d^{\prime} \in \llbracket d \rrbracket \\
\begin{subarray}{c}{\mathbf{d} \in(\mathbb{Z} \geq 0)^{N} \\
|\mathbf{d}|=d-d^{\prime}{ d ^ { \prime } \in \llbracket d \rrbracket \\
\begin{subarray} { c } { \mathbf { d } \in ( \mathbb { Z } \geq 0 ) ^ { N } \\
| \mathbf { d } | = d - d ^ { \prime } } }\end{subarray}} \sum_{\mathbf{p} \in \llbracket n \rrbracket^{N}} \sum_{\substack{\mathbf{b}^{\prime} \in\left(\mathbb{Z}^{\geq 0}\right)^{N} \\
b_{s}^{\prime} \leq b_{s} \forall s}} \mathcal{C}_{g ; \mathbf{p}, \mathbf{b}^{\prime}}^{\left(d^{\prime}\right)} \prod_{s=1}^{s=N} \llbracket \mathcal{Z}_{p_{s}}\left(\hbar_{s}, \mathbf{x}_{s}, q\right) \rrbracket_{\hbar_{s}^{-1}, q ; b_{s}-b_{s}^{\prime}, d_{s}},
\end{aligned}
$$

where $\llbracket \mathcal{Z}_{p}(\hbar, \mathbf{x}, q) \rrbracket_{\hbar^{-1}, q ; b, d^{\prime}}$ is the coefficient of $q^{d^{\prime}}\left(\hbar^{-1}\right)^{b}$ in

$$
\mathcal{Z}_{p}(\hbar, \mathbf{x}, q) \in H_{\mathbb{T}}^{*}\left(\mathbb{P}^{n-1}\right)\left[\left[\hbar^{-1}, q\right]\right] .
$$

Since $H_{\mathbb{T}}^{*}\left(\mathbb{P}^{n-1}\right)$ and $H_{\mathbb{T}}^{*}\left(\mathbb{P}_{N}^{n-1}\right)$ are free modules over $\mathbb{Q}[\alpha]$ with bases $\left\{\mathbf{x}^{p}\right\}_{p \in\lfloor n \rrbracket}$ and $\left\{\underline{\mathbf{x}}^{\mathbf{p}}\right\}_{\mathbf{p} \in\left\lfloor n \rrbracket^{N}\right.}$, respectively, and

$$
\llbracket \mathcal{Z}_{p}(\hbar, \mathbf{x}, q) \rrbracket_{\hbar^{-1}, q ; b, d^{\prime}} \in H_{\mathbb{T}}^{*}\left(\mathbb{P}^{n-1}\right), \quad \llbracket \mathcal{Z}^{(g)}(\underline{\hbar}, \underline{\mathbf{x}}, q) \rrbracket_{\underline{\hbar}^{-1}, q ; \mathbf{b}+\mathbf{1}, d} \in H_{\mathbb{T}}^{*}\left(\mathbb{P}_{N}^{n-1}\right)
$$

by (3.9) and (3.8), respectively, (4.1) and induction on $d$ imply that $\mathcal{C}_{g ; \mathbf{p}, \mathbf{b}}^{(d)} \in \mathbb{Q}[\alpha]$.

By [20, Lemma 3.3], the ideal $\mathcal{I} \subset \mathbb{Q}[\alpha]^{S_{n}}$ does not contain any power of $\sigma_{n} D_{\alpha}$. Along with the algebraic independence of elementary symmetric polynomials $\sigma_{1}, \ldots, \sigma_{n}$, this implies the second claim of the lemma. 
For $g \in \mathbb{Z}^{\geq 0}$ and a finite set $S$ with $2 g+|S| \geq 3$, we denote by $\overline{\mathcal{M}}_{g, S}$ the Deligne-Mumford moduli space of stable $S$-marked genus $g$ curves and by

$$
\mathbb{E}_{g} \longrightarrow \overline{\mathcal{M}}_{g, S}
$$

the Hodge vector bundle of holomorphic differentials. For each $f \in S$, let

$$
L_{f} \longrightarrow \overline{\mathcal{M}}_{g, S}
$$

be the universal tangent line bundle for the marked point labeled by $f$.

For $g \in \mathbb{Z}^{\geq 0}$, a finite set $S$, and $d \in \mathbb{Z}^{+}$, we denote by $\overline{\mathfrak{M}}_{g, S}\left(\mathbb{P}^{n-1}, d\right)$ the moduli space of stable $S$-marked genus $g$ degree $d$ maps to $\mathbb{P}^{n-1}$. For each $f \in S$, let

$$
\psi_{f} \equiv c_{1}\left(L_{f}^{*}\right) \in H^{2}\left(\overline{\mathfrak{M}}_{g, S}\left(\mathbb{P}^{n-1}, d\right)\right)
$$

be the first Chern class of the universal cotangent line bundle $L_{f}$ over $\overline{\mathfrak{M}}_{g, S}\left(\mathbb{P}^{n-1}, d\right)$ for the marked point labeled by $f$.

For an $[n]$-valued $N$-marked graph $\bar{\Gamma}$ as in (3.24), let

$$
\mathcal{A}(\bar{\Gamma})=\left(\mathbb{Z}^{\geq 0}\right)^{\overline{\mathrm{Fl}}(\bar{\Gamma})} \times \prod_{v \in \overline{\mathrm{Ver}}}\left(\mathbb{Z}^{\geq 0}\right)^{\overline{\mathfrak{g}}(v)} .
$$

Let $\Gamma$ be a connected $[n]$-valued $N$-marked genus $g$ weighted graph as in (3.21) and $\bar{\Gamma}$ be its core as in (3.24). Define

$$
\begin{aligned}
& \mathrm{Fl}(\Gamma)=\bigsqcup_{v \in \overline{\mathrm{Ver}}} \mathrm{Fl}_{v}(\Gamma), \quad \overline{\mathrm{Fl}}(\Gamma)=\bigsqcup_{v \in \overline{\mathrm{Ver}}} \overline{\mathrm{Fl}}_{v}(\Gamma)=\left([N] \cap \eta^{-1}(\overline{\mathrm{Ver}})\right) \sqcup \mathrm{Fl}(\Gamma), \\
& \mathrm{Fl}^{*}(\Gamma)=\left([N]-\eta^{-1}(\overline{\mathrm{Ver}})\right) \sqcup \overline{\mathrm{Edg}}, \quad \overline{\mathrm{Fl}}^{*}(\Gamma)=[N] \sqcup \overline{\mathrm{Edg}}=\left([N] \cap \eta^{-1}(\overline{\mathrm{Ver}})\right) \sqcup \mathrm{Fl}^{*}(\Gamma), \\
& \mathcal{A}^{\star}(\Gamma)=\left\{\left(\mathbf{b},\left(I_{v}\right)_{v \in \overline{\mathrm{Ver}}}\right) \in\left(\mathbb{Z}^{\geq 0}\right)^{\overline{\mathrm{Fl}}(\Gamma)} \times \prod_{v \in \overline{\mathrm{Ver}}}\left(\mathbb{Z}^{\geq 0}\right)^{g_{v}}:|\mathbf{b}|_{\overline{\mathrm{Fl}}_{v}(\Gamma)}=\mu_{g_{v}}\left(I_{v}\right)+\left|\overline{\mathrm{Fl}}_{v}(\Gamma)\right| \forall v \in \overline{\mathrm{Ver}}\right\} .
\end{aligned}
$$

For $v \in \overline{\operatorname{Ver}}$ and $I \in\left(\mathbb{Z}^{\geq 0}\right)^{g_{v}}$, let

$$
\mathcal{A}_{v ; I}^{\star}(\Gamma)=\left\{\mathbf{b} \in\left(\mathbb{Z}^{\geq 0}\right)^{\overline{\mathrm{Fl}}_{v}(\Gamma)}:|\mathbf{b}|=\mu_{g_{v}}(I)+\left|\overline{\mathrm{Fl}}_{v}(\Gamma)\right|\right\} .
$$

We denote the components of an element $(\mathbf{b}, \mathbf{I})$ of $\mathcal{A}^{\star}(\Gamma)$ by $b_{f} \in \mathbb{Z}^{\geq 0}$ for $f \in \overline{\mathrm{Fl}}(\Gamma)$ and $I_{v} \in\left(\mathbb{Z}^{\geq 0}\right)^{g_{v}}$

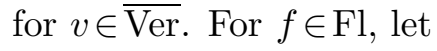

$$
\mu(f)=\mu(\eta(f)) \in[n] \text { and } \mu_{c}(f)=\mu\left(\eta\left(f^{\prime}\right)\right) \in[n] \text { if } e_{f}=\left\{f, f^{\prime}\right\}
$$

be the $\mu$-values at the two flags contained in the edge $e_{f}$.

\subsection{The closed formula approach}

We first break $\Gamma$ and $\mathcal{Z}_{\Gamma}$ at all vertices $v$ of $\overline{\operatorname{Ver}} \subset$ Ver as described in Section 3.3. The set of strands of type (S2) is naturally indexed by the edges $\overline{\operatorname{Edg}}$ of $\bar{\Gamma}$. By the constructions of the core and of the strands $\Gamma_{\mathfrak{p}}$ in Section 3.3 .

$$
f_{e}^{ \pm} \in \mathrm{Fl}_{\eta\left(f_{e}^{ \pm}\right)}(\Gamma) \quad \forall e \equiv\left\{f_{e}^{+}, f_{e}^{-}\right\} \in \overline{\operatorname{Edg}}
$$


and the set $S_{e}$ of the marked points on the strand $\Gamma_{e}$ corresponding to an edge $e$ as above is $\left\{\widehat{f}_{e}^{+}, \widehat{f}_{e}^{-}\right\}$. The set of strands of type (S3) is naturally indexed by the subset $[N]-\eta^{-1}(\overline{\mathrm{Ver}})$ of the original marked points. By the construction of the core in Section 3.3, for each such $s \in[N]$ there exists a unique flag $f_{s} \in \mathrm{Fl}_{\bar{\eta}(s)}(\Gamma)$ so that the edge $e_{f_{s}} \in \operatorname{Edg}$ splits the graph $\Gamma$ into two, one containing the vertex $\bar{\eta}(s)$ and the other containing the vertex $\eta(s)$. The set $S_{s}$ of the marked points on the corresponding strand $\Gamma_{s}$ is $\left\{s, \widehat{f}_{s}\right\}$. All of the flags $f_{s}$ with $s \in[N]-\eta^{-1}(\overline{\mathrm{Ver}})$ and $f_{e}^{+}, f_{e}^{-}$with $e \in \overline{\operatorname{Edg}}$ are distinct. The set of strands of type (S1) is naturally indexed by the complement

$$
\mathrm{Fl}^{\prime}(\Gamma) \equiv \mathrm{Fl}(\Gamma)-\left\{f_{s}: s \in[N]-\eta^{-1}(\overline{\mathrm{Ver}})\right\}-\left\{f_{e}^{ \pm}: e \in \overline{\mathrm{Edg}}\right\} \subset \mathrm{Fl}
$$

of such flags inside of all flags of $\Gamma$ at the vertices $\overline{\mathrm{Ver}} \subset$ Ver. The set $S_{f}$ of the marked points on the strand $\Gamma_{f}$ corresponding to $f \in \mathrm{Fl}^{\prime}(\Gamma)$ is $\{\widehat{f}\}$.

The set

$$
\mathrm{Fl}^{\dagger}(\Gamma) \equiv \mathrm{Fl}^{*}(\Gamma) \sqcup \mathrm{Fl}^{\prime}(\Gamma)
$$

of all strands $\Gamma_{\mathfrak{p}}$ of $\Gamma$ is thus a quotient of $\mathrm{Fl}(\Gamma)$ so that two flags $f_{1}, f_{2} \in \mathrm{Fl}(\Gamma)$ determine the same element $f_{1}^{\dagger}=f_{2}^{\dagger}$ of $\mathrm{Fl}^{\dagger}(\Gamma)$ if and only if the marked points labeled by $\widehat{f}_{1}$ and $\widehat{f}_{2}$ lie on the same strand. The set

$$
\overline{\mathrm{Fl}}^{\dagger}(\Gamma) \equiv\left([N] \cap \eta^{-1}(\overline{\mathrm{Ver}})\right) \sqcup \mathrm{Fl}^{\dagger}(\Gamma)=[N] \sqcup \overline{\operatorname{Edg}^{\prime}} \sqcup \mathrm{Fl}^{\prime}(\Gamma)
$$

is similarly a quotient of the set $\overline{\mathrm{Fl}}(\Gamma)$. For $\mathfrak{p} \in \overline{\mathrm{Fl}}^{\dagger}(\Gamma)$, we write $f \in \mathfrak{p}$ if $f \in \overline{\mathrm{Fl}}(\Gamma)$ and $f^{\dagger}=\mathfrak{p}$. Denote by $S_{\mathfrak{p}}^{*} \subset[N]$ the empty set if $\mathfrak{p} \notin[N]$ and $\{\mathfrak{p}\}$ if $\mathfrak{p} \in[N]$. For each $v \in \overline{\mathrm{Ver}}$, let

$$
\mathrm{Fl}_{v}^{\prime}(\Gamma) \equiv \mathrm{Fl}_{v}(\Gamma) \cap \mathrm{Fl}^{\prime}(\Gamma)
$$

be the subset of strands of type (S1) arising from $v$.

The fixed locus $\mathcal{Z}_{\Gamma}$ corresponding to $\Gamma$ and the Euler class of the virtual normal bundle of $\mathcal{Z}_{\Gamma}$ are given by

$$
\begin{gathered}
\mathcal{Z}_{\Gamma}=\prod_{v \in \overline{\mathrm{Ver}}} \overline{\mathcal{M}}_{g_{v}, \overline{\mathrm{Fl}}_{v}(\Gamma)} \times \prod_{\mathfrak{p} \in \mathrm{Fl}^{\dagger}(\Gamma)} \mathcal{Z}_{\Gamma_{\mathfrak{p}}}, \\
\frac{\prod_{v \in \overline{\mathrm{Ver}}} \mathbf{e}\left(T_{P_{\mu(v)}} \mathbb{P}^{n-1}\right)}{\mathbf{e}\left(\mathcal{N} \mathcal{Z}_{\Gamma}^{\text {vir }}\right)}=\prod_{v \in \overline{\mathrm{Ver}}} \mathbf{e}\left(\mathbb{E}_{g_{v}}^{*} \otimes T_{P_{\mu(v)}} \mathbb{P}^{n-1}\right) \prod_{\mathfrak{p} \in \mathrm{Fl}^{\dagger}(\Gamma)} \frac{1}{\mathbf{e}\left(\mathcal{N} \mathcal{Z}_{\Gamma_{\mathfrak{p}}}\right)} \prod_{f \in \mathrm{Fl}(\Gamma)} \frac{\mathbf{e}\left(T_{P_{\mu(f)}} \mathbb{P}^{n-1}\right)}{\hbar_{f}^{\prime}-\psi_{\widehat{f}}},
\end{gathered}
$$

where

$$
\hbar_{f}^{\prime} \equiv c_{1}\left(L_{f}\right) \in H^{*}\left(\overline{\mathcal{M}}_{g_{v}, \overline{F l}_{v}(\Gamma)}\right) \quad \forall f \in \mathrm{Fl}_{v}(\Gamma), v \in \overline{\operatorname{Ver}} .
$$

By [11, Section 27.2],

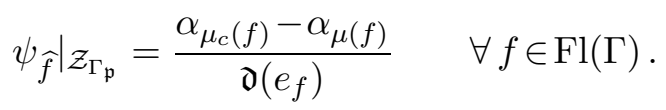


Thus,

$$
\begin{aligned}
& \int_{\overline{\mathcal{M}}_{g_{v}, \overline{\mathrm{F}}_{v}(\Gamma)}} \lambda_{g_{v} ; I}\left\{\left(\prod_{f \in \mathrm{Fl}_{v}(\Gamma)} \frac{1}{\hbar_{f}^{\prime}-\psi_{\widehat{f}}}\right)\left(\prod_{s \in S_{v}} \frac{1}{\hbar_{s}-\psi_{s}}\right)\right\} \\
& =(-1)^{\left|\mathrm{Fl}_{v}(\Gamma)\right|} \sum_{\mathbf{b} \in(\mathbb{Z} \geq 0)^{\mathrm{FI}_{v}(\Gamma)}} \int_{\overline{\mathcal{M}}_{g_{v}, \overline{\mathrm{F}}_{v}(\Gamma)}} \lambda_{g_{v} ; I}\left\{\left(\prod_{f \in \mathrm{Fl}_{v}(\Gamma)} \psi_{\widehat{f}}^{-b_{f}-1} \hbar_{f}^{\prime b_{f}}\right)\left(\prod_{s \in S_{v}} \hbar_{s}^{-b_{s}-1} \psi_{s}^{b_{s}}\right)\right\} \\
& =\sum_{\mathbf{b} \in \mathcal{A}_{v ; I}^{\star}(\Gamma)}\left\{\left\langle\left\langle\lambda_{g_{v} ; I} ; \tau_{\mathbf{b}}\right\rangle\right\rangle\left(\prod_{f \in \mathrm{Fl}_{v}(\Gamma)}\left(\frac{\alpha_{\mu(f)}-\alpha_{\mu_{c}(f)}}{\mathfrak{d}\left(e_{f}\right)}\right)^{-b_{f}-1}\right)\left(\prod_{s \in S_{v}} \hbar_{s}^{-b_{s}-1}\right)\right\}
\end{aligned}
$$

for all $I \in\left(\mathbb{Z}^{\geq 0}\right)^{g_{v}}$ and $v \in \overline{\text { Ver. }}$

Combining (4.4)-(4.6) with (3.15), (3.35), and (3.16), we obtain

$$
\begin{aligned}
\left(\prod_{v \in \overline{\operatorname{Ver}}} \prod_{k \neq \mu(v)}\left(\alpha_{\mu(v)}-\alpha_{k}\right)\right) \int_{\mathcal{Z}_{\Gamma}} \frac{1}{\mathbf{e}\left(\mathcal{N} \mathcal{Z}_{\Gamma}^{\text {vir }}\right)} \prod_{s=1}^{s=N} \frac{\mathrm{ev}_{s}^{*} \phi_{i_{s}}}{\hbar_{s}-\psi_{s}} \\
=\sum_{(\mathbf{b}, \mathbf{I}) \in \mathcal{A}^{\star}(\Gamma)}\left\{\prod_{v \in \overline{\operatorname{Ver}}}\left(C_{g_{v}, n ; I_{v}} \alpha_{\mu(v)}^{(n-1) g_{v}-\left\|I_{v}\right\|}+h_{g_{v} ; n ; I_{v}}\left(\alpha_{\mu(v)}\right)\right)\left\langle\left\langle\lambda_{g_{v} ; I_{v}} ; \widetilde{\tau}_{\mathbf{b}}\right\rangle\right\rangle\right. \\
\left.\quad \times \prod_{\mathfrak{p} \in \overline{\mathrm{Fl}}^{\dagger}(\Gamma)}\left(\prod_{f \in \mathfrak{p}} \frac{1}{b_{f} !}\left(\frac{\alpha_{\mu(f)}-\alpha_{\mu_{c}(f)}}{\mathfrak{d}\left(e_{f}\right)}\right)^{-b_{f}-1} \int_{\mathcal{Z}_{\Gamma_{\mathfrak{p}}}} \frac{\left.\prod_{f \in \mathfrak{p}}^{\mathbf{e}\left(\mathcal{N e v}_{f}^{*} \phi_{\mu}(f)\right.} \prod_{\Gamma_{\mathfrak{p}}}\right)}{\prod_{s \in S_{\mathfrak{p}}^{*}}} \frac{\mathrm{ev}_{s}^{*} \phi_{i_{s}}}{\hbar_{s}-\psi_{s}}\right)\right\}
\end{aligned}
$$

where

$$
\prod_{f \in \mathfrak{p}} \frac{1}{b_{f} !}\left(\frac{\alpha_{\mu(f)}-\alpha_{\mu_{c}(f)}}{\mathfrak{d}\left(e_{f}\right)}\right)^{-b_{f}-1} \int_{\mathcal{Z}_{\Gamma_{\mathfrak{p}}}} \frac{\prod_{f \in \mathfrak{p}} \mathbf{e}\left(\mathcal{N} \mathcal{Z}_{\Gamma_{\mathfrak{p}}}^{*} \phi_{\mu(f)}\right.}{\prod_{s \in S_{\mathfrak{p}}^{*}}} \frac{\mathrm{ev}_{s}^{*} \phi_{i_{s}}}{\hbar_{s}} \equiv \frac{1}{b_{\mathfrak{p}} !}\left(\hbar_{\mathfrak{p}}^{-b_{\mathfrak{p}}-1} \prod_{k \neq i_{\mathfrak{p}}}\left(\alpha_{\mu(\eta(\mathfrak{p}))}-\alpha_{k}\right)\right)
$$

if $\mathfrak{p} \in[N] \cap \eta^{-1}(\overline{\mathrm{Ver}}) \subset \overline{\mathrm{Fl}}^{\dagger}(\Gamma) \cap \overline{\mathrm{Fl}}(\Gamma)$. The equality in (4.7) holds after taking into account the automorphism groups; this is done below after summing over all possibilities for the strands $\Gamma_{\mathfrak{p}}$.

Lemma 4.2. If $\mathfrak{p} \in \mathrm{Fl}^{\prime}(\Gamma)$, then

$$
\begin{aligned}
\sum_{\Gamma_{\mathfrak{p}}} q^{\left|\Gamma_{\mathfrak{p}}\right|} \prod_{f \in \mathfrak{p}}\left(\frac{\alpha_{\mu(f)}-\alpha_{\mu_{c}(f)}}{\mathfrak{d}\left(e_{f}\right)}\right)^{-b_{f}-1} \int_{\mathcal{Z}_{\Gamma_{\mathfrak{p}}}} \frac{\prod_{f \in \mathfrak{p}} \mathbf{e}\left(\mathcal{N} \mathcal{Z}_{\Gamma_{\mathfrak{p}}}^{*} \phi_{\mu(f)}\right.}{\prod_{s \in S_{\mathfrak{p}}^{*}}} \frac{\mathrm{ev}_{s}^{*} \phi_{i_{s}}-\psi_{s}}{} \\
=\underset{\substack{\mathfrak{R}=0 \\
\hbar_{\mathfrak{p}}=0}}{\mathfrak{R}}\left\{\left(-\hbar_{\mathfrak{p}}\right)^{-b_{\mathfrak{p}}} \widetilde{\mathcal{Z}}^{*}\left(\hbar_{\mathfrak{p}}, \alpha_{v}^{\prime}, q\right)\right\}
\end{aligned}
$$

where the sum is taken over all possibilities for the strand $\Gamma_{\mathfrak{p}}$, leaving the vertex $v \equiv \eta(\mathfrak{p})$, with $\alpha_{v}^{\prime} \equiv \alpha_{\mu(v)}$ fixed.

Proof. In this case, $S_{\mathfrak{p}}^{*}=\emptyset$ and each $\Gamma_{\mathfrak{p}}$ is a connected 1-marked genus 0 graph. Thus, the claim of this lemma is [20, (2.14)] with $\mathbf{e}\left(\mathcal{V}_{0}^{\prime}\right)=1$ and slightly different notation (the left-hand side of [20, $(2.14)]$ is also missing $\left.q^{\left|\Gamma_{e}\right|}\right)$. 
Lemma 4.3. If $\mathfrak{p} \in[N]$, then

$$
\begin{aligned}
\sum_{\Gamma_{\mathfrak{p}}} q^{\left|\Gamma_{\mathfrak{p}}\right|} \prod_{f \in \mathfrak{p}}\left(\frac{\alpha_{\mu(f)}-\alpha_{\mu_{c}(f)}}{\mathfrak{d}\left(e_{f}\right)}\right)^{-b_{f}-1} \int_{\mathcal{Z}_{\Gamma_{\mathfrak{p}}}} \frac{\prod_{f \in \mathfrak{p}} \mathbf{e}\left(\mathcal{N} \mathcal{Z}_{\Gamma_{\mathfrak{p}}}^{*} \phi_{\mu(f)}\right.}{\prod_{s \in S_{\mathfrak{p}}^{*}}} \frac{\mathrm{ev}_{s}^{*} \phi_{i_{s}}}{\hbar_{s}-\psi_{s}} \\
=-\underset{\hbar_{f}=0}{\mathfrak{R}}\left\{\left(-\hbar_{f}\right)^{-b_{f}-1} \mathcal{Z}\left(\hbar_{f}, \hbar_{\mathfrak{p}}, \alpha_{v}^{\prime}, \alpha_{i_{\mathfrak{p}}}, q\right)\right\}
\end{aligned}
$$

where the sum is taken over

- all possibilities for the strand $\Gamma_{\mathfrak{p}}$, leaving the vertex $v \equiv \bar{\eta}(\mathfrak{p})$, with $\alpha_{v}^{\prime} \equiv \alpha_{\mu(v)}$ fixed and $\left|\Gamma_{\mathfrak{p}}\right| \in \mathbb{Z}^{+}$, and carrying the marked point indexed by $\mathfrak{p}$,

- and the special case $\Gamma_{\mathfrak{p}}$ corresponding to the case $\eta(\mathfrak{p})=v$.

Proof. In this case, $S_{\mathfrak{p}}^{*}=\{\mathfrak{p}\}, \eta(f)=v$ for the unique element $f \in \mathfrak{p}$, and each $\Gamma_{\mathfrak{p}}$ is a connected 2 -marked genus 0 graph. There is a unique edge $e \equiv e_{f_{\mathfrak{p}}}$ from the vertex $v \equiv \bar{\eta}(\mathfrak{p})$ which is contained in $\Gamma_{\mathfrak{p}}$. By (3.24) and Lemma 1.2 in [21] with $m=2$ and $\mathbf{e}\left(\mathcal{V}_{0}^{\prime \prime}\right)=1$, the left-hand side of (4.9) summed over $\Gamma_{\mathfrak{p}}$ with $\mathfrak{d}(e)=d$ for $d \in \mathbb{Z}^{+}$and $\mu_{c}\left(f_{\mathfrak{p}}\right)=j$ for $j \in[n]-\mu(v)$ is the residue of

$$
\left(-\hbar_{f}\right)^{-b_{f}-1} \mathcal{Z}^{*}\left(\hbar_{f}, \hbar_{\mathfrak{p}}, \alpha_{v}^{\prime}, \alpha_{i_{\mathfrak{p}}}, q\right) \mathrm{d} \hbar_{f} \in \mathbb{Q}_{\alpha}\left(\hbar_{f}, \hbar_{\mathfrak{p}}\right)[[q]] \mathrm{d} \hbar_{f}
$$

at $\hbar_{f}=\left(\alpha_{j}-\alpha_{v}^{\prime}\right) / d$. Furthermore, this meromorphic 1-form on $\mathbb{P}^{1}$ has no poles outside of $\hbar_{f}=\left(\alpha_{j}-\alpha_{v}^{\prime}\right) / d$ with $d \in \mathbb{Z}^{+}$and $j \in[n]-\mu(v)$ and $\hbar=0, \infty$.

By [18, (3.10)] with $l=0$ and $\langle\mathbf{a}\rangle=1$,

$$
\mathcal{Z}\left(\hbar_{f}, \hbar_{\mathfrak{p}}, \alpha_{v}^{\prime}, \alpha_{i_{\mathfrak{p}}}, q\right)=-\frac{1}{\hbar_{f}+\hbar_{\mathfrak{p}}} \sum_{\substack{p_{-}, p_{+}, r \in \mathbb{Z} \geq 0 \\ p_{-}+p_{+}+r=n-1}} \widehat{\sigma}_{r} \mathcal{Z}_{p_{-}}\left(\hbar_{f}, \alpha_{v}^{\prime}, q\right) \mathcal{Z}_{p_{+}}\left(\hbar_{\mathfrak{p}}, \alpha_{i_{\mathfrak{p}}}, q\right)
$$

By [18, (3.11)], $\hbar_{f}^{-2} \mathcal{Z}_{p_{-}}\left(\hbar_{f}, \alpha_{v}^{\prime}, q\right) \mathrm{d} \hbar_{f}$ has no pole at $\hbar_{f}=\infty$. Combining these statements with the Residue Theorem on $\mathbb{P}^{1}$, we find that

$$
\begin{aligned}
\sum_{\Gamma_{\mathfrak{p}}} q^{\left|\Gamma_{\mathfrak{p}}\right|} \prod_{f \in \mathfrak{p}}\left(\frac{\alpha_{\mu(f)}-\alpha_{\mu_{c}(f)}}{\mathfrak{d}\left(e_{f}\right)}\right)^{-b_{f}-1} \int_{\mathcal{Z}_{\Gamma_{\mathfrak{p}}}} \frac{\prod_{f \in \mathfrak{p}} \mathbf{e}\left(\mathcal{N} \mathcal{Z}_{\Gamma_{\mathfrak{p}}}^{*} \phi_{\mu(f)}\right.}{\prod_{s \in S_{\mathfrak{p}}^{*}} \frac{\mathrm{ev}_{s}^{*} \phi_{i_{s}}}{\hbar_{s}-\psi_{s}}} \\
=-\underset{\substack{\mathfrak{R} \\
\hbar_{f}=0}}{\mathfrak{R}}\left\{\left(-\hbar_{f}\right)^{-b_{f}-1_{\mathcal{Z}}}\left(\hbar_{f}, \hbar_{\mathfrak{p}}, \alpha_{v}^{\prime}, \alpha_{i_{\mathfrak{p}}}, q\right)\right\}
\end{aligned}
$$

if the sum is taken over all possibilities for the strand $\Gamma_{\mathfrak{p}}$, leaving the vertex $v \equiv \bar{\eta}(\mathfrak{p})$, with $\alpha_{v}^{\prime} \equiv \mu(v)$ fixed and $\left|\Gamma_{\mathfrak{p}}\right| \in \mathbb{Z}^{+}$, and carrying the marked point indexed by $\mathfrak{p}$.

By the definition of the degree 0 term in the $(g, m)=(0,2)$ case of (3.8),

$$
\hbar_{\mathfrak{p}}^{-b_{\mathfrak{p}}-1} \prod_{k \neq i_{\mathfrak{p}}}\left(\alpha_{v}^{\prime}-\alpha_{k}\right)=(-1)^{b_{\mathfrak{p}}} \underset{\substack{\hbar_{f}=0 \\ \mathfrak{R}}}{\mathfrak{n}}\left\{\hbar_{f}^{-b_{\mathfrak{p}}-1} \llbracket \mathcal{Z}\left(\hbar_{f}, \hbar_{\mathfrak{p}}, \alpha_{v}^{\prime}, \alpha_{i_{\mathfrak{p}}}, q\right) \rrbracket_{q ; 0}\right\} .
$$

This is the summand for the special case $\Gamma_{\mathfrak{p}}$ corresponding to the case $\eta(\mathfrak{p})=v$. 
Lemma 4.4. If $\mathfrak{p} \in \overline{\mathrm{Edg}}$, then

$$
\begin{aligned}
\sum_{\Gamma_{\mathfrak{p}}} q^{\left|\Gamma_{\mathfrak{p}}\right|} & \prod_{f \in \mathfrak{p}}\left(\frac{\alpha_{\mu(f)}-\alpha_{\mu_{c}(f)}}{\mathfrak{d}\left(e_{f}\right)}\right)^{-b_{f}-1} \int_{\mathcal{Z}_{\Gamma_{\mathfrak{p}}}} \frac{\prod_{f \in \mathfrak{p}} \mathrm{ev}_{f}^{*} \phi_{\mu(f)}}{\mathbf{e}\left(\mathcal{N} \mathcal{Z}_{\Gamma_{\mathfrak{p}}}\right)} \prod_{s \in S_{\mathfrak{p}}^{*}} \frac{\mathrm{ev}_{s}^{*} \phi_{i_{s}}}{\hbar_{s}-\psi_{s}} \\
& =\underset{\substack{\mathfrak{R} \\
\hbar_{+}=0}}{\mathfrak{R}}\left\{\underset{\hbar_{-}=0}{\mathfrak{R}}\left\{\left(-\hbar_{-}\right)^{-b_{\mathfrak{p}}^{-}-1}\left(-\hbar_{+}\right)^{-b_{\mathfrak{p}}^{+}-1} \mathcal{Z}\left(\hbar_{-}, \hbar_{+}, \alpha_{\mathfrak{p}}^{-}, \alpha_{\mathfrak{p}}^{+}, q\right)\right\}\right\},
\end{aligned}
$$

where $b_{\mathfrak{p}}^{ \pm} \equiv b_{f^{ \pm}}$if $\mathfrak{p}=\left\{f^{+}, f^{-}\right\}$and the sum is taken over all possibilities for the strand $\Gamma_{\mathfrak{p}}$ between the vertices $v_{-} \equiv \bar{\eta}\left(f_{\mathfrak{p}}^{-}\right)$and $v_{+} \equiv \bar{\eta}\left(f_{\mathfrak{p}}^{+}\right)$, with $\alpha_{\mathfrak{p}}^{-} \equiv \mu\left(v_{-}\right)$and $\alpha_{\mathfrak{p}}^{+} \equiv \mu\left(v_{+}\right)$fixed.

Proof. By the reasoning as in the proof of Lemma 4.3 applied twice,

$$
\begin{aligned}
\sum_{\Gamma_{\mathfrak{p}}} q^{\left|\Gamma_{\mathfrak{p}}\right|} & \prod_{f \in \mathfrak{p}}\left(\frac{\alpha_{\mu(f)}-\alpha_{\mu_{c}(f)}}{\mathfrak{d}\left(e_{f}\right)}\right)^{-b_{f}-1} \int_{\mathcal{Z}_{\Gamma_{\mathfrak{p}}}} \frac{\prod_{f \in \mathfrak{p}} \mathbf{e v} \mathrm{ev}_{f}^{*} \phi_{\mu(f)}}{\mathbf{e}\left(\mathcal{N} \mathcal{Z}_{\Gamma_{\mathfrak{p}}}\right)} \prod_{s \in S_{\mathfrak{p}}^{*}} \frac{\mathrm{ev}_{s}^{*} \phi_{i_{s}}}{\hbar_{s}-\psi_{s}} \\
= & \underset{\hbar_{+}=0}{\mathfrak{R}}\left\{\underset{\hbar_{-}=0}{\mathfrak{R}}\left\{\left(-\hbar_{-}\right)^{-b_{\mathfrak{p}}^{-}-1}\left(-\hbar_{+}\right)^{-b_{\mathfrak{p}}^{+}-1} \mathcal{Z}^{*}\left(\hbar_{-}, \hbar_{+}, \alpha_{\mathfrak{p}}^{-}, \alpha_{\mathfrak{p}}^{+}, q\right)\right\}\right\} .
\end{aligned}
$$

Since

$$
\underset{\hbar_{+}=0}{\mathfrak{R}}\left\{\underset{\hbar_{-}=0}{\mathfrak{R}}\left\{\hbar_{-}^{-b_{\mathfrak{p}}^{-}-1} \hbar_{+}^{-b_{\mathfrak{p}}^{+}-1} \llbracket \mathcal{Z}\left(\hbar_{-}, \hbar_{+}, \alpha_{\mathfrak{p}}^{-}, \alpha_{\mathfrak{p}}^{+}, q\right) \rrbracket_{q ; 0}\right\}\right\}=0 \quad \forall b_{\mathfrak{p}}^{-}, b_{\mathfrak{p}}^{+} \in \mathbb{Z}^{\geq 0},
$$

we can replace $\mathcal{Z}^{*}$ in the previous expression by $\mathcal{Z}$.

We now combine (4.7) with (4.3) and Lemmas 4.24 4.4 and sum over all possibilities for $\mathrm{Fl}^{\prime}(\Gamma)$. Taking into account the automorphism groups, we obtain

$$
\begin{aligned}
|\operatorname{Aut}(|\bar{\Gamma}|)|\left(\prod_{v \in \overline{\operatorname{Ver}}} \prod_{k \neq \mu(v)}\left(\alpha_{v}^{\prime}-\alpha_{k}\right)\right) \sum_{\Gamma} q^{|\Gamma|} \int_{\mathcal{Z}_{\Gamma}} \frac{1}{\mathbf{e}\left(\mathcal{N} \mathcal{Z}_{\Gamma}^{\mathrm{vir}}\right)} \prod_{s=1}^{s=N} \frac{\mathrm{ev}_{s}^{*} \phi_{i_{s}}}{\hbar_{s}-\psi_{s}} \\
=\sum_{(\mathbf{b}, \mathbf{I}) \in \mathcal{A}(\bar{\Gamma})}\left\{\prod_{v \in \overline{\operatorname{Ver}}} \widetilde{\mathcal{Z}}_{\mathbf{b}_{v}, I_{v}}^{\left(g_{v}\right)}\left(\alpha_{v}^{\prime}, q\right) \prod_{s \in[N]} \underset{\hbar=0}{\mathfrak{R}}\left\{\frac{(-\hbar)^{-b_{s}}}{b_{s} ! \hbar} \mathcal{Z}\left(\hbar, \hbar_{s}, \alpha_{\bar{\eta}(s)}^{\prime}, \alpha_{i_{s}}, q\right)\right\}\right. \\
\quad \times \prod_{e \in \overline{\operatorname{Edg}}} \underset{\hbar_{+}^{\mathfrak{R}}=0}{\mathfrak{R}}\left\{\underset{\hbar_{-}=0}{\mathfrak{R}}\left\{\frac{\left(-\hbar_{-}\right)^{-b_{e}^{-}}\left(-\hbar_{+}\right)^{-b_{e}^{+}}}{b_{e}^{-} ! b_{e}^{+} ! \hbar_{-} \hbar_{+}} \mathcal{Z}\left(\hbar_{-}, \hbar_{+}, \alpha_{e}^{-}, \alpha_{e}^{+}, q\right)\right\}\right\},
\end{aligned}
$$

with $\widetilde{\mathcal{Z}}_{\mathbf{b}_{v}, I_{v}}^{\left(g_{v}\right)}$ as in (3.36). The sum on the left-hand side above is taken over all equivalence classes of connected $[n]$-valued $N$-marked genus $g$ weighted graphs $\Gamma$ as in (3.21) with a fixed core $\bar{\Gamma}$ as in (3.24).

By the first statement of Lemma 5.1 ,

$$
\begin{aligned}
& (-1)^{\mu_{g_{v}}\left(I_{v}\right)+m_{v}-c} \zeta\left(\alpha_{v}^{\prime}, q\right)^{\left|\mathbf{b}_{v}\right|-\left(\mu_{g_{v}}\left(I_{v}\right)+m_{v}-c\right)}\left(\begin{array}{c}
\left|\mathbf{b}_{v}\right|-\left|\underline{\epsilon}_{v}\right| \\
\mu_{g_{v}}\left(I_{v}\right)+m_{v}-c-\left|\underline{\epsilon}_{v}\right|
\end{array}\right) \prod_{f \in \overline{\mathrm{FI}}_{v}(\bar{\Gamma})}\left(\begin{array}{c}
b_{f} \\
\epsilon_{f}
\end{array}\right) \\
& =\sum_{\substack{\mathbf{b}_{v}^{\prime} \in(\mathbb{Z} \geq 0) \overline{\mathrm{F}}_{v}(\bar{\Gamma}) \\
\left|\mathbf{b}_{v}^{\prime}\right|+\left|\underline{\epsilon}_{v}\right|+c=\mu_{g v}\left(I_{v}\right)+m_{v} \\
b_{f}^{\prime}+\epsilon_{f} \leq b_{f} \forall f \in \overline{\mathrm{Fl}}_{v}(\bar{\Gamma})}} \prod_{\substack{\left.f \bar{\Gamma}_{v}(\bar{\Gamma}) \\
\forall f\right)^{b_{f}} b_{f} !}}\left(\frac{\left(-\zeta\left(\alpha_{v}^{\prime}, q\right)\right)^{b_{f}-b_{f}^{\prime}-\epsilon_{f}}}{\epsilon_{f} ! b_{f}^{\prime} !}\right)
\end{aligned}
$$


for all $c \in \mathbb{Z}$ and $\mathbf{b}, \underline{\epsilon} \in\left(\mathbb{Z}^{\geq 0}\right)^{\overline{\mathrm{Fl}}(\bar{\Gamma})}$, where $m_{v}=\left|\overline{\mathrm{Fl}}_{v}(\bar{\Gamma})\right|$. Combining (4.13) with (3.37), we thus obtain

$$
\begin{aligned}
|\operatorname{Aut}(|\bar{\Gamma}|)| & \left(\prod_{v \in \overline{\mathrm{Ver}}} \prod_{k \neq \mu(v)}\left(\alpha_{v}^{\prime}-\alpha_{k}\right)\right) \sum_{\Gamma} q^{|\Gamma|} \int_{\mathcal{Z}_{\Gamma}} \frac{1}{\mathbf{e}\left(\mathcal{N} \mathcal{Z}_{\Gamma}^{\mathrm{vir}}\right)} \prod_{s=1}^{s=N} \frac{\mathrm{ev}_{s}^{*} \phi_{i_{s}}}{\hbar_{s}-\psi_{s}} \\
= & \sum_{\left(\mathbf{b}^{\prime}, \underline{\epsilon}, \mathbf{c}, \mathbf{I}\right) \in \widetilde{\mathcal{A}}^{\star}(\bar{\Gamma})}\left\{\prod_{v \in \overline{\operatorname{Ver}}} \Psi_{I_{v} ; \mathbf{c}_{v}}^{\left(g_{v}, \underline{c}_{v}\right)}\left(\alpha_{v}^{\prime}, q\right) \times \prod_{s=1}^{s=N}\left(\frac{1}{b_{s}^{\prime} !} \mathfrak{\Re = 0}\left\{\frac{e^{-\frac{\zeta\left(\alpha_{\bar{\eta}}^{\prime}(s), q\right)}{\hbar}}}{\hbar^{b_{s}^{\prime}+\epsilon_{s}+1}} \mathcal{Z}\left(\hbar, \hbar_{s}, \alpha_{\bar{\eta}(s)}^{\prime}, \alpha_{i_{s}}, q\right)\right\}\right)\right. \\
& \left.\times \prod_{e \in \overline{\mathrm{Edg}}}\left(\frac{1}{b_{e}^{\prime-} ! b_{e}^{\prime+} !} \underset{\hbar_{+}=0}{\mathfrak{R}}\left\{\underset{\hbar_{-}^{\mathfrak{R}}=0}{\mathfrak{R}}\left\{\frac{e^{-\frac{\zeta\left(\alpha_{e}^{-}, q\right)}{\hbar_{-}}-\frac{\zeta\left(\alpha_{e}^{+}, q\right)}{\hbar_{+}}}}{\hbar_{-}^{b_{e}^{\prime-}+\epsilon_{e}^{-}+1} \hbar_{+}^{b_{e}^{\prime+}+\epsilon_{e}^{+}+1}} \mathcal{Z}\left(\hbar_{-}, \hbar_{+}, \alpha_{e}^{-}, \alpha_{e}^{+}, q\right)\right\}\right\}\right)\right\},
\end{aligned}
$$

where $b_{e}^{\prime \pm}=b_{f^{ \pm}}^{\prime}$ and $\epsilon_{e}^{\prime \pm}=\epsilon_{f^{ \pm}}^{\prime}$ if $e=\left\{f^{+}, f^{-}\right\}$and $\widetilde{\mathcal{A}}^{\star}(\bar{\Gamma})$ is as in (2.26).

The first statement of Lemma 3.2 and Corollary 3.3 reduce the last two products above to

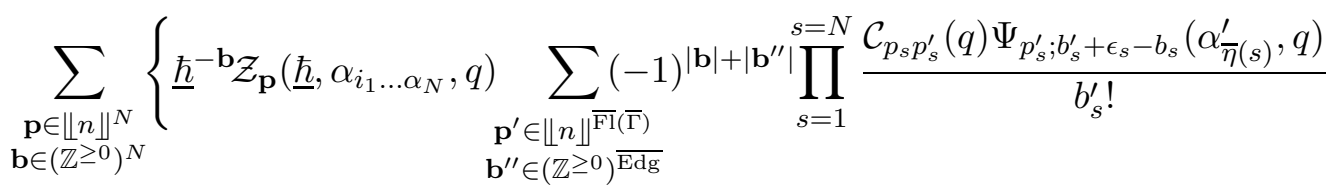

$$
\begin{aligned}
& \left.\times \prod_{e \in \overline{\mathrm{Edg}}} \frac{\mathcal{C}_{p_{e}^{\prime} p_{e}^{\prime+}}(q) \Psi_{p_{e}^{\prime-} ; b_{e}^{\prime-}+\epsilon_{e}^{-}-b_{e}^{\prime \prime}}\left(\alpha_{e}^{-}, q\right) \Psi_{p_{e}^{\prime+} ; b_{e}^{\prime+}+\epsilon_{e}^{+}+1+b_{e}^{\prime \prime}}\left(\alpha_{e}^{+}, q\right)}{b_{e}^{\prime-} ! b_{e}^{\prime+} !}\right\} .
\end{aligned}
$$

By the Residue Theorem on $\mathbb{P}^{1}$,

$$
\begin{array}{r}
\sum_{j=1}^{n} \frac{\Psi_{I_{v} ; \mathbf{c}_{v}}^{\left(g_{v}\right)}\left(\alpha_{j}, q\right)}{\prod_{k \neq j}\left(\alpha_{j}-\alpha_{k}\right)} \prod_{s \in \bar{\eta}^{-1}(v)} \frac{\Psi_{p_{s}^{\prime} ; b_{s}^{\prime}+\epsilon_{s}-b_{s}}\left(\alpha_{j}, q\right)}{b_{s}^{\prime} !} \prod_{f \in \mathrm{Fl}_{v}^{-}(\bar{\Gamma})} \frac{\Psi_{p_{f}^{\prime} ; b_{f}^{\prime}+\epsilon_{f}-b_{e_{f}}^{\prime \prime}}\left(\alpha_{j}, q\right)}{b_{f}^{\prime} !} \prod_{f \in \mathrm{Fl}_{v}^{+}(\bar{\Gamma})} \frac{\Psi_{p_{f}^{\prime} ; b_{f}^{\prime}+\epsilon_{f}+1+b_{e_{f}}^{\prime \prime}}\left(\alpha_{j}, q\right)}{b_{f}^{\prime} !} \\
=-\underset{\mathbf{x}=0, \infty}{\mathfrak{R}}\left\{\frac{\mathcal{F}_{I_{v} ; \mathbf{c}_{v} ; \mathbf{p}_{v}^{\prime}, \mathbf{b}_{v}^{\prime}, \mathbf{b}_{v}^{\prime \prime}(\mathbf{x}, q)}^{k=n}}{\left.\prod_{k=1}^{\left(g_{j}, \epsilon_{v}\right)} \alpha_{k}\right)}\right\},
\end{array}
$$

where

$$
\begin{aligned}
& \mathcal{F}_{I_{v} ; \mathbf{c}_{v} ; \mathbf{p}_{v}^{\prime}, \mathbf{b}_{v}^{\prime}, \mathbf{b}_{v}^{\prime \prime}}^{\left(g_{v}, \epsilon_{v}\right)}(\mathbf{x}, q)=\Psi_{I_{v} ; \mathbf{c}_{v}}^{\left(g_{v}, \epsilon_{v}\right)}(\mathbf{x}, q) \prod_{s \in \bar{\eta}^{-1}(v)} \frac{\Psi_{p_{s}^{\prime} ; b_{s}^{\prime}+\epsilon_{s}-b_{s}}(\mathbf{x}, q)}{b_{s}^{\prime} !} \prod_{f \in \mathrm{Fl}_{v}^{-}(\bar{\Gamma})} \frac{\Psi_{p_{f}^{\prime} ; b_{f}^{\prime}+\epsilon_{f}-b_{e_{f}^{\prime \prime}}}(\mathbf{x}, q)}{b_{f}^{\prime} !} \\
& \times \prod_{f \in \mathrm{Fl}_{v}^{+}(\bar{\Gamma})} \frac{\Psi_{p_{f}^{\prime} ; b_{f}^{\prime}+\epsilon_{f}+1+b_{e_{f}}^{\prime \prime}}(\mathbf{x}, q)}{b_{f}^{\prime} !}
\end{aligned}
$$

We now divide both sides of (4.14) by the first two factors on the left-hand side and sum over all possibilities for $\mu(v) \in[n]$ and $\bar{\Gamma} \in \mathcal{A}_{g, N}$; we replace $\bar{\Gamma}$ with $\Gamma$ below. Using the equations after (4.14), 
we obtain an explicit formula for the coefficients $\mathcal{C}_{g ; \mathbf{p}, \mathbf{b}}^{(d)}$ in Theorem B,

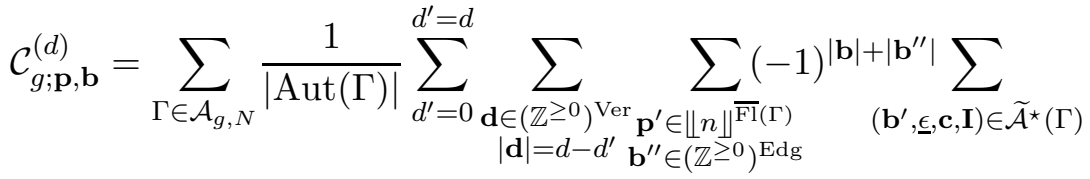

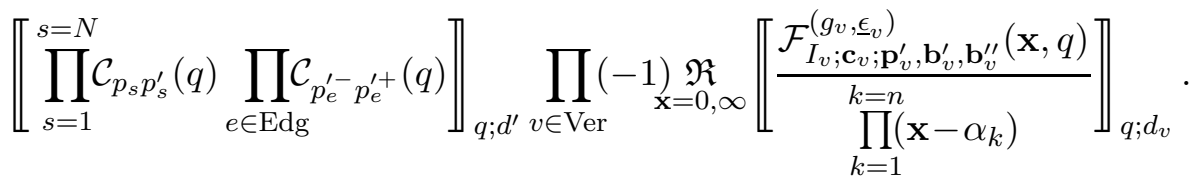

This establishes (3.11).

It remains to show that (3.12) with the summation over $t \in \mathbb{Z}$ instead of $\mathbb{Z}^{\geq 0}$ holds for some $\mathrm{c}_{g ; \mathbf{p}, \mathbf{b}}^{(d, t)} \in \mathbb{Q}$ such that $\mathrm{c}_{g ; \mathbf{p}, \mathbf{b}}^{(d, 0)}=\mathrm{c}_{g ; \mathbf{p}, \mathbf{b}}^{(d)}$ with $\mathrm{c}_{g ; \mathbf{p}, \mathbf{b}}^{(d)}$ given by (2.29) . Let $\left(\mathbf{b}^{\prime}, \underline{\epsilon}, \mathbf{c}, \mathbf{I}\right) \in \widetilde{\mathcal{A}}^{\star}(\bar{\Gamma})$ and $\mathbf{p}, \mathbf{p}^{\prime}, \mathbf{b}, \mathbf{b}^{\prime \prime}$ be as above. For $v \in \overline{\mathrm{Ver}}$, define

$$
\left|\mathbf{p}_{v}^{\prime}\right|=\sum_{f \in \overline{\mathrm{Fl}}_{v}(\bar{\Gamma})} p_{f}^{\prime}, \quad\left|\mathbf{b}_{v}\right|=\sum_{s \in \bar{\eta}^{-1}(v)} b_{s}, \quad\left|\mathbf{b}_{v}^{\prime \prime-}\right|=\sum_{f \in \mathrm{Fl}_{v}^{-}(\bar{\Gamma})} b_{e_{f}}^{\prime \prime}, \quad\left|\mathbf{b}_{v}^{\prime \prime+}\right|=\sum_{f \in \mathrm{Fl}_{v}^{+}(\bar{\Gamma})} b_{e_{f}}^{\prime \prime}
$$

By (3.34),

$$
\prod_{s=1}^{s=N} \mathcal{C}_{p_{s} p_{s}^{\prime}}(q) \prod_{e \in \overline{\mathrm{Edg}}} \mathcal{C}_{p_{e}^{\prime-}} p_{e}^{\prime+}(q) \sim \begin{cases}1, & \text { if } p_{s}+p_{s}^{\prime}, p_{e}^{\prime-}+p_{e}^{\prime+}=n-1 \forall s \in[N], e \in \overline{\mathrm{Edg}} ; \\ 0 & \text { otherwise. }\end{cases}
$$

By (3.38), (3.32), and (2.26),

$$
\begin{array}{r}
\llbracket \mathcal{F}_{I_{v} ; \mathbf{c}_{v} ; \mathbf{p}_{v}^{\prime}, \mathbf{b}_{v}^{\prime}, \mathbf{b}_{v}^{\prime \prime}}^{\left(g_{v}, \epsilon^{\prime}\right.}(\mathbf{x}, q) \rrbracket_{q ; d_{v}} \sim \mathbf{x}^{(n-1)-n d_{v}+(n-4)\left(g_{v}-1\right)-\mid \overline{\mathrm{FI}}}(\bar{\Gamma})|-| \mathrm{Fl}_{v}^{+}(\bar{\Gamma})|+| \mathbf{p}_{v}^{\prime}|+| \mathbf{b}_{v}|+| \mathbf{b}_{v}^{\prime \prime-}|-| \mathbf{b}_{v}^{\prime \prime+} \mid \\
\times \llbracket F_{I_{v} ; \mathbf{c}_{v} ; \mathbf{p}_{v}^{\prime}, \mathbf{b}_{v}^{\prime}, \mathbf{b}_{v}^{\prime \prime}}^{\left(g_{v} \epsilon_{i}\right)} \rrbracket_{q ; d_{v}},
\end{array}
$$

where

$$
F_{I_{v} ; \mathbf{c}_{v} ; \mathbf{p}_{v}^{\prime}, \mathbf{b}_{v}^{\prime}, \mathbf{b}_{v}^{\prime \prime}}^{\left(g_{v}, \epsilon^{\prime}\right.}(q)=\Phi_{I_{v} ; \mathbf{c}_{v}}^{\left(g_{v}, \epsilon_{v}\right)}(q) \prod_{s \in \bar{\eta}^{-1}(v)} \frac{\Phi_{p_{s}^{\prime} ; b_{s}^{\prime}+\epsilon_{s}-b_{s}}(q)}{b_{s}^{\prime} ! \Phi_{0}(q)} \prod_{f \in \mathrm{F}_{v}^{-}(\bar{\Gamma})} \frac{\Phi_{p_{f}^{\prime} ; b_{f}^{\prime}+\epsilon_{f}-b_{e_{f}}^{\prime \prime}}(q)}{b_{f}^{\prime} ! \Phi_{0}(q)} \prod_{f \in \mathrm{Fl}_{v}^{+}(\bar{\Gamma})} \frac{\Phi_{p_{f}^{\prime} ; b_{f}^{\prime}+\epsilon_{f}+1+b_{e_{f}}^{\prime \prime}}(q)}{b_{f}^{\prime} ! \Phi_{0}(q)} .
$$

Along with the last two statements in Lemma 3.1, this implies that

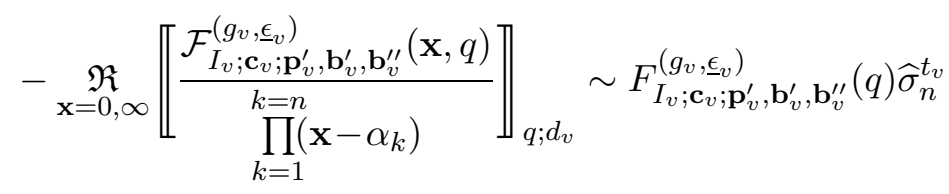

with $t_{v} \in \mathbb{Z}$ defined by

$$
\left|\mathbf{p}_{v}^{\prime}\right|+\left|\mathbf{b}_{v}\right|+\left|\mathbf{b}_{v}^{\prime \prime-}\right|-\left|\mathbf{b}_{v}^{\prime \prime+}\right|-\left|\mathrm{Fl}_{v}^{+}(\bar{\Gamma})\right|=(n-4)\left(1-g_{v}\right)+\left|\overline{\mathrm{Fl}}_{v}(\bar{\Gamma})\right|+n\left(d_{v}+t_{v}\right)
$$

if an integer $t_{v}$ satisfying the above condition does not exist, we define $\widehat{\sigma}_{n}^{t_{v}}$ to be 0 . 
By (4.15), the above paragraph, and the middle statement in Lemma 3.1 ,

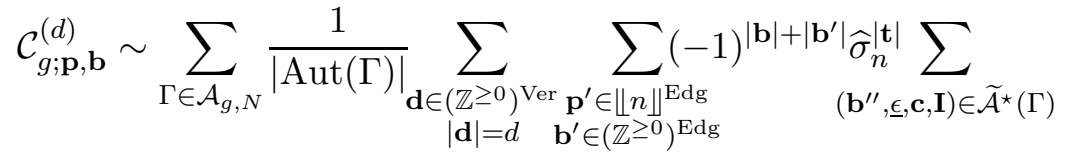

$$
\begin{aligned}
& \prod_{v \in \operatorname{Ver}} \llbracket \Phi_{I_{v} ; \mathbf{c}_{v}}^{\left(g_{v}, \epsilon_{v}\right)}(q) \prod_{s \in S_{v}} \frac{\Phi_{\widehat{p}_{s} ; b_{s}^{\prime \prime}+\epsilon_{s}-b_{s}}(q)}{b_{s}^{\prime \prime} ! \Phi_{0}(q)} \prod_{f \in \mathrm{Fl}_{v}^{-}(\Gamma)} \frac{\Phi_{p_{e_{f}}^{\prime} ; b_{f}^{\prime \prime}+\epsilon_{f}-b_{e_{f}}^{\prime}}(q)}{b_{f}^{\prime \prime} ! \Phi_{0}(q)} \prod_{f \in \mathrm{Fl}_{v}^{+}(\Gamma)} \frac{\Phi_{\widehat{p}_{e_{f}}^{\prime} ; b_{f}^{\prime \prime}+\epsilon_{f}+1+b_{e_{f}}^{\prime}}(q)}{b_{f}^{\prime \prime} ! \Phi_{0}(q)} \|_{q ; d_{v}}
\end{aligned}
$$

with $\mathbf{t} \equiv\left(t_{v}\right)_{v \in \text { Ver }} \in \mathbb{Z}^{\text {Ver }}$ defined by

$$
\begin{aligned}
\sum_{s \in S_{v}}\left(\widehat{p}_{s}+b_{s}\right)+ & \sum_{f \in \mathrm{Fl}_{v}^{-}(\Gamma)}\left(p_{e_{f}}^{\prime}+b_{e_{f}}^{\prime}\right)+\sum_{f \in \mathrm{Fl}_{v}^{+}(\Gamma)}\left(\widehat{p}_{e_{f}}^{\prime}-1-b_{e_{f}}^{\prime}\right) \\
& =(n-4)\left(1-g_{v}\right)+\left|\overline{\mathrm{Fl}}_{v}(\Gamma)\right|+n\left(d_{v}+t_{v}\right) \quad \forall v \in \text { Ver; }
\end{aligned}
$$

the corresponding summand above is taken to be 0 if an integer $t_{v}$ satisfying the above condition does not exist for some $v \in$ Ver. This confirms (3.12) with $\mathrm{c}_{g ; \mathbf{p}, \mathbf{b}}^{(d)} \equiv \mathrm{c}_{g ; \mathbf{p}, \mathbf{b}}^{(d, 0)}$ as defined in (2.29) (and describes $\mathrm{c}_{g ; \mathbf{p}, \mathbf{b}}^{(d, t)}$ with $t \in \mathbb{Z}^{+}$as well).

\subsection{The recursion approach}

We next show that (3.12) holds with the coefficients $c_{g ; \mathbf{p}, \mathbf{b}}^{(d, t)}$ as defined recursively at the end of Section 2.3 . Let $\Gamma$ be a connected $[n]$-valued $N$-marked genus $g$ weighted graph and $\bar{\Gamma}$ be its core as before. This time we break $\Gamma$ only at the vertex

$$
v \equiv \bar{\eta}(N) \in \overline{\mathrm{Ver}} \subset \mathrm{Ver}
$$

into strands $\Gamma_{\mathfrak{p}}$ as in (3.25). Each edge $e \equiv\left\{f, f^{\prime}\right\}$ of the original graph $\Gamma$ with $\eta(f)=v$ keeps a copy $v_{f}$ of the vertex $v$ satisfying (3.26) and carrying an additional marked point labeled by $\widehat{f}$. The set of strands (i.e. of the connected components of the graph) obtained from $\Gamma$ in this way is indexed by a quotient $\mathrm{Fl}_{v}^{\dagger}(\Gamma)$ of the set $\mathrm{Fl}_{v}(\Gamma)$ of flags of $\Gamma$ at $v$ so that $f_{1}, f_{2} \in \mathrm{Fl}_{v}(\Gamma)$ determine the same element $f_{1}^{\dagger}=f_{2}^{\dagger}$ of $\mathrm{Fl}_{v}^{\dagger}(\Gamma)$ if and only if the marked points labeled by $\widehat{f}_{1}$ and $\widehat{f}_{2}$ lie on the same strand. Since the strands $\Gamma_{\mathfrak{p}}$ are obtained from the genus $g$ graph $\Gamma$ by breaking all edges at $v$,

$$
g_{v}+\sum_{\mathfrak{p} \in \mathrm{Fl}_{v}^{\dagger}(\Gamma)} \mathfrak{a}\left(\Gamma_{\mathfrak{p}}\right)+\left|\mathrm{Fl}_{v}(\Gamma)\right|=g+\left|\mathrm{Fl}_{v}^{\dagger}(\Gamma)\right| .
$$

The set of strands of type (S1) is now the subset

$$
\mathrm{Fl}_{v}^{\prime}(\Gamma) \equiv \mathrm{Fl}^{\prime}(\Gamma) \cap \mathrm{Fl}_{v}(\Gamma)
$$

of the set of strands of type (S1) in Section 4.2 that leave from the vertex $v$. With the notation as in (4.2), the set $\mathrm{Fl}_{v}^{*}(\Gamma)$ of the remaining strands $\mathfrak{p}$ is a quotient of the set

$$
\mathrm{Fl}_{v}(\Gamma)-\mathrm{Fl}_{v}^{\prime}(\Gamma) \equiv\left\{f_{s}: s \in[N], \bar{\eta}(s)=v \neq \eta(s)\right\} \sqcup \mathrm{Fl}_{v}(\bar{\Gamma}) .
$$

With $S_{v} \equiv[N] \cap \eta^{-1}(v)$ as before, let

$$
\overline{\mathrm{Fl}}_{v}^{*}(\Gamma) \equiv S_{v} \sqcup \mathrm{Fl}_{v}^{*}(\Gamma)
$$


The set

$$
\overline{\mathrm{Fl}}_{v}^{\dagger}(\Gamma) \equiv S_{v} \sqcup \mathrm{Fl}_{v}^{\dagger}(\Gamma)=S_{v} \sqcup \mathrm{Fl}_{v}^{*}(\Gamma) \sqcup \mathrm{Fl}^{\prime}(\Gamma)=\overline{\mathrm{Fl}}_{v}^{*}(\Gamma) \sqcup \mathrm{Fl}^{\prime}(\Gamma)
$$

is similarly a quotient of the set $\overline{\mathrm{Fl}}_{v}(\Gamma)$. For $\mathfrak{p} \in \overline{\mathrm{Fl}}_{v}^{\dagger}(\Gamma)$, we write $f \in \mathfrak{p}$ if $f \in \overline{\mathrm{Fl}}_{v}(\Gamma)$ and $f^{\dagger}=\mathfrak{p}$.

For $s \in S_{v}$, define

$$
\mathfrak{a}\left(\Gamma_{s}\right)=0, \quad S_{s}^{*}=\{s\}, \quad|s|=\left|S_{s}^{\prime}\right|=1
$$

For $\mathfrak{p} \in \mathrm{Fl}_{v}^{\dagger}(\Gamma)$, let

$$
S_{\mathfrak{p}}^{*} \equiv S_{\mathfrak{p}} \cap[N], \quad S_{\mathfrak{p}}^{\prime} \equiv S_{\mathfrak{p}}-[N]=\left\{\widehat{f}: f^{\dagger}=\mathfrak{p}\right\}, \quad \text { and } \quad|\mathfrak{p}| \equiv\left|S_{\mathfrak{p}}^{\prime}\right|
$$

be the set of the original marked points carried by $\mathfrak{p}$, the set of the additional marked points, and the cardinality of the latter, respectively. Thus,

$$
\begin{aligned}
& |\mathfrak{p}| \in \mathbb{Z}^{+} \quad \forall \mathfrak{p} \in \overline{\mathrm{Fl}}^{\dagger}(\Gamma), \quad \mathrm{Fl}_{v}^{\prime}(\Gamma)=\left\{\mathfrak{p} \in \overline{\mathrm{Fl}}^{\dagger}(\Gamma): \mathfrak{a}\left(\Gamma_{\mathfrak{p}}\right)=0, S_{\mathfrak{p}}^{*}=\emptyset,|\mathfrak{p}|=1\right\}, \\
& {[N]-S_{v}=\bigsqcup_{\mathfrak{p} \in \mathrm{Fl}^{*}(\Gamma)} S_{\mathfrak{p}}^{*}, \quad \mathrm{Fl}_{v}(\Gamma)-\mathrm{Fl}_{v}^{\prime}(\Gamma)=\bigsqcup_{\mathfrak{p} \in \mathrm{Fl}^{*}(\Gamma)} S_{\mathfrak{p}}^{\prime}, \quad g_{v}+\sum_{\mathfrak{p} \in \overline{\mathrm{Fl}}_{v}^{*}(\Gamma)}\left(\mathfrak{a}\left(\Gamma_{\mathfrak{p}}\right)+|\mathfrak{p}|\right)=g+\left|\overline{\mathrm{Fl}}_{v}^{*}(\Gamma)\right| .}
\end{aligned}
$$

By the choice of $v$, either $N \in S_{v}$ or $S_{\mathfrak{p}}=\{N\}$ for some $\mathfrak{p} \in \mathrm{Fl}_{v}^{*}(\Gamma)$ with $\mathfrak{a}\left(\Gamma_{\mathfrak{p}}\right)=0$ and $|\mathfrak{p}|=1$. Thus,

$$
\left(g_{v},\left(\mathfrak{a}\left(\Gamma_{\mathfrak{p}}\right), S_{\mathfrak{p}}^{*},|\mathfrak{p}|\right)_{\mathfrak{p} \in \overline{\mathrm{Fl}}_{v}^{*}(\Gamma)}\right) \in \mathcal{P}_{g, N}^{\left(\left|\overline{\mathrm{Fl}}_{v}^{*}(\Gamma)\right|\right)}
$$

The analogues of the decompositions (4.4) and (4.5) in this case are

$$
\begin{gathered}
\mathcal{Z}_{\Gamma}=\overline{\mathcal{M}}_{g_{v}, \overline{\mathrm{Fl}}_{v}(\Gamma)} \times \prod_{\mathfrak{p} \in \mathrm{Fl}_{v}^{\dagger}(\Gamma)} \mathcal{Z}_{\Gamma_{\mathfrak{p}}} \\
\frac{\mathbf{e}\left(T_{P_{\mu(v)}} \mathbb{P}^{n-1}\right)}{\mathbf{e}\left(\mathcal{N} \mathcal{Z}_{\Gamma}^{\mathrm{vir}}\right)}=\mathbf{e}\left(\mathbb{E}_{g_{v}}^{*} \otimes T_{P_{\mu(v)}} \mathbb{P}^{n-1}\right) \prod_{\mathfrak{p} \in \mathrm{Fl} l_{v}^{\dagger}(\Gamma)} \frac{1}{\mathbf{e}\left(\mathcal{N} \mathcal{Z}_{\Gamma_{\mathfrak{p}}}^{\mathrm{vir}}\right)} \prod_{f \in \mathrm{Fl} l_{v}(\Gamma)} \frac{\mathbf{e}\left(T_{P_{\mu(v)}} \mathbb{P}^{n-1}\right)}{\hbar_{f}^{\prime}-\psi_{\widehat{f}}}
\end{gathered}
$$

For each $I \in\left(\mathbb{Z}^{\geq 0}\right)^{g_{v}}$, (4.6) still applies. The analogue of (4.7) is now

$$
\begin{aligned}
& \prod_{k \neq \mu(v)}\left(\alpha_{\mu(v)}-\alpha_{k}\right) \int_{\mathcal{Z}_{\Gamma}} \frac{1}{\mathbf{e}\left(\mathcal{N} \mathcal{Z}_{\Gamma}^{\text {vir }}\right)} \prod_{s=1}^{s=N}\left(\frac{\mathrm{ev}_{s}^{*} \phi_{i_{s}}}{\hbar_{s}-\psi_{s}}\right) \\
& =\sum_{I \in(\mathbb{Z} \geq 0)^{g_{v}}} \sum_{\mathbf{b} \in \mathcal{A}_{v ; I}^{\star}(\Gamma)}\left\{\left(C_{g_{v}, n ; I} \alpha_{\mu(v)}^{(n-1) g_{v}-\|I\|}+h_{g_{v} ; n ; I}\left(\alpha_{\mu(v)}\right)\right)\left\langle\left\langle\lambda_{g_{v} ; I} ; \widetilde{\tau}_{\mathbf{b}}\right\rangle\right\rangle\right. \\
& \left.\times \prod_{\mathfrak{p} \in \overline{\mathrm{Fl}}_{v}^{\dagger}(\Gamma)}\left(\prod_{f \in \mathfrak{p}} \frac{1}{b_{f} !}\left(\frac{\alpha_{\mu(v)}-\alpha_{\mu_{c}(f)}}{\mathfrak{d}\left(e_{f}\right)}\right)^{-b_{f}-1} \int_{\mathcal{Z}_{\Gamma_{\mathfrak{p}}}} \frac{\prod_{f \in \mathfrak{p}} \mathbf{e}\left(\mathcal{N} \mathcal{Z}_{f}^{*} \phi_{\Gamma_{\mathfrak{p}}}^{\text {vir }}\right)}{\prod_{s \in S_{\mathfrak{p}}^{*}}} \frac{\mathrm{ev}_{s}^{*} \phi_{i_{s}}}{\hbar_{s}-\psi_{s}}\right)\right\},
\end{aligned}
$$

where

$$
\prod_{f \in \mathfrak{p}} \frac{1}{b_{f} !}\left(\frac{\alpha_{\mu(v)}-\alpha_{\mu_{c}(f)}}{\mathfrak{d}\left(e_{f}\right)}\right)^{-b_{f}-1} \int_{\mathcal{Z}_{\Gamma_{\mathfrak{p}}}} \frac{\prod_{f \in \mathfrak{p}} \mathbf{e}\left(\mathcal{N} \mathcal{Z}_{\Gamma_{\mathfrak{p}}}^{*} \phi_{\mu}\right)}{\prod_{s \in S_{\mathfrak{p}}^{*}}} \prod_{\hbar_{s}-\psi_{s}}^{\mathrm{ev}_{s}^{*} \phi_{i_{s}}} \equiv \frac{1}{b_{\mathfrak{p}} !}\left(\hbar_{\mathfrak{p}}^{-b_{\mathfrak{p}}-1} \prod_{k \neq i_{\mathfrak{p}}}\left(\alpha_{\mu(v)}-\alpha_{k}\right)\right)
$$


if $\mathfrak{p} \in S_{v} \subset \overline{\mathrm{Fl}}_{v}^{\dagger}(\Gamma) \cap \overline{\mathrm{Fl}}_{v}(\Gamma)$. The equality in (4.19) holds after taking into account the automorphism groups; this is done below after summing over all possibilities for the strands $\Gamma_{\mathfrak{p}}$.

The relevant sum over all possibilities for the strands $\Gamma_{\mathfrak{p}}$ with $\mathfrak{p} \in \mathrm{Fl}_{v}^{\prime}(\Gamma)$ is described by (4.8) with $\mu(f)=\mu(v)$. For $\mathfrak{p} \in \overline{\mathrm{Fl}}_{v}^{*}(\Gamma)$ such that $\mathfrak{a}\left(\Gamma_{\mathfrak{p}}\right)=0,|\mathfrak{p}|=1$, and $S_{\mathfrak{p}}=\left\{s_{\mathfrak{p}}\right\}$ for some $s_{\mathfrak{p}} \in \bar{\eta}^{-1}(v)$, the corresponding sum is described by (4.9) with $\mu(f)=\mu(v), \hbar_{\mathfrak{p}}=\hbar_{s_{\mathfrak{p}}}$, and $i_{\mathfrak{p}}=i_{s_{\mathfrak{p}}}$. Lemma 4.5] below extends (4.9) to the remaining cases. For each $\mathfrak{p} \in \overline{\mathrm{Fl}}_{v}^{*}(\Gamma)$, we order the elements of $S_{\mathfrak{p}}^{\prime} \subset \mathrm{Fl}_{v}(\Gamma)$ as $f_{1}, \ldots, f_{|\mathfrak{p}|}$ and define

$$
\underset{\left(\hbar_{f}=0\right)_{f \in \mathfrak{p}}}{\mathfrak{R}}\{\cdots\}=\underset{\hbar_{f_{|\mathfrak{p}|}}=0}{\mathfrak{R}}\left\{\ldots \underset{\hbar_{f_{1}}=0}{\mathfrak{R}}\{\ldots\} \ldots\right\} .
$$

Lemma 4.5. If $\mathfrak{p} \in \overline{\mathrm{Fl}}_{v}^{*}(\Gamma)$ with $2\left(\mathfrak{a}\left(\Gamma_{\mathfrak{p}}\right)+|\mathfrak{p}|\right)+\left|S_{\mathfrak{p}}^{*}\right|>3$, then

$$
\begin{aligned}
& \sum_{\Gamma_{\mathfrak{p}}} q^{\left|\Gamma_{\mathfrak{p}}\right|} \prod_{f \in \mathfrak{p}}\left(\frac{\alpha_{\mu(v)}-\alpha_{\mu_{c}(f)}}{\mathfrak{d}\left(e_{f}\right)}\right)^{-b_{f}-1} \int_{\mathcal{Z}_{\Gamma_{\mathfrak{p}}}} \frac{\prod_{f \in \mathfrak{p}} \mathbf{e}\left(\mathcal{N} \mathcal{Z}_{\Gamma_{\mathfrak{p}}}^{*} \phi_{\mu(f)}\right)}{\prod_{s \in S_{\mathfrak{p}}^{*}}} \frac{\mathrm{ev}_{s}^{*} \phi_{i_{s}}}{\hbar_{s}-\psi_{s}} \\
& =(-1)^{|\mathfrak{p}|} \underset{\left(\hbar_{f}=0\right)_{f \in \mathfrak{p}}}{\mathfrak{R}}\left\{\prod_{f \in \mathfrak{p}}\left(-\hbar_{f}\right)^{-b_{f}-1} \mathcal{Z}^{\left(\mathfrak{a}\left(\Gamma_{\mathfrak{p}}\right)\right)}\left(\left(\hbar_{f}\right)_{f \in S_{\mathfrak{p}}},\left(\alpha_{v}^{\prime}\right)_{f \in \mathfrak{p}},\left(\alpha_{i_{f}}\right)_{f \in S_{\mathfrak{p}}^{*}}, q\right)\right\},
\end{aligned}
$$

where the sum is taken over all possibilities for the strand $\Gamma_{\mathfrak{p}}$ with $v=\bar{\eta}(f)$ for all $f \in S_{\mathfrak{p}}^{\prime}$ and $\alpha_{v}^{\prime} \equiv \alpha_{\mu(v)}$ fixed.

Proof. If $\mathfrak{a}\left(\Gamma_{\mathfrak{p}}\right)=0,|\mathfrak{p}|=2$, and $S_{\mathfrak{p}}^{*}=\emptyset$, (4.20) is the case of (4.12) with $\mu(f)=\mu(v)$ and $\alpha_{\mathfrak{p}}^{ \pm}=\alpha_{v}^{\prime}$. Suppose $2 \mathfrak{a}\left(\Gamma_{\mathfrak{p}}\right)+|\mathfrak{p}|+\left|S_{\mathfrak{p}}^{*}\right| \geq 3$. By the proofs of (3.24) and Lemma 1.2 in [21] applied with arbitrary genus and $\mathbf{e}\left(\mathcal{V}_{0}^{\prime \prime}\right)=1$, the left-hand side of (4.20) summed over $\Gamma_{\mathfrak{p}}$ with $\mathfrak{d}\left(e_{f}\right)=d_{f}$ for $d_{f} \in \mathbb{Z}^{+}$and $\mu_{c}(f)=j_{f}$ for $j_{f} \in[n]-\mu(v)$ fixed for each $f \in \mathfrak{p}$ is the $|\mathfrak{p}|$-fold residue of

$$
\prod_{f \in \mathfrak{p}}\left(-\hbar_{f}\right)^{-b_{f}-1} \mathcal{Z}^{\left(\mathfrak{a}\left(\Gamma_{\mathfrak{p}}\right)\right)}\left(\left(\hbar_{f}\right)_{f \in S_{\mathfrak{p}}},\left(\alpha_{v}^{\prime}\right)_{f \in \mathfrak{p}},\left(\alpha_{i_{f}}\right)_{f \in S_{\mathfrak{p}}^{*}}, q\right)
$$

at $\hbar_{f}=\left(\alpha_{j_{f}}-\alpha_{v}^{\prime}\right) / d_{f}$ for each $f \in \mathfrak{p}$ (i.e. first take the residue of the above power series in rational functions at $\hbar_{f_{1}}=\left(\alpha_{j_{1}}-\alpha_{v}^{\prime}\right) / d_{f_{1}}$, then the residue of the resulting power series at $\hbar_{f_{1}}=\left(\alpha_{j_{f_{1}}}-\alpha_{v}^{\prime}\right) / d_{f_{1}}$, and so on). Furthermore, for each $f \in \mathfrak{p}$ the associated meromorphic 1-form on $\mathbb{P}^{1}$ in $\hbar_{f}$ has no poles outside of $\hbar_{f}=\left(\alpha_{j}-\alpha_{v}^{\prime}\right) / d$ with $d \in \mathbb{Z}^{+}$and $j \in[n]-\mu(v)$ and $\hbar_{f}=0$. The claim now follows from the Residue Theorem on $\mathbb{P}^{1}$, as in the proof of Lemma 4.3, but applied $|\mathfrak{p}|$ times.

We now combine (4.19) with Lemmas 4.2, 4.3, and 4.5 and sum over all possibilities for $\mathrm{Fl}_{v}^{\prime}(\Gamma)$. Taking into account the automorphism groups, we obtain an analogue (4.13):

$$
\begin{aligned}
\prod_{k \neq \mu(v)}\left(\alpha_{v}^{\prime}-\alpha_{k}\right) \sum_{\Gamma} q^{|\Gamma|} \int_{\mathcal{Z}_{\Gamma}} \frac{1}{\mathbf{e}\left(\mathcal{N} \mathcal{Z}_{\Gamma}^{\text {vir }}\right)} \prod_{s=1}^{s=N} \frac{\mathrm{ev}_{s}^{*} \phi_{i_{s}}}{\hbar_{s}-\psi_{s}}=\sum_{I \in(\mathbb{Z} \geq 0))^{g v}} \sum_{\mathbf{b} \in \mathcal{A}_{v ; I}^{\star}(\Gamma)}\left\{\widetilde{\mathcal{Z}}_{\mathbf{b}, I}^{\left(g_{v}\right)}\left(\alpha_{v}^{\prime}, q\right)\right. \\
\times \prod_{\mathfrak{p} \in \overline{\mathrm{F}}_{v}^{*}(\Gamma)} \frac{(-1)^{|\mathfrak{p}|}}{|\mathfrak{p}| !\left(\hbar_{f}=0\right)_{f \in \mathfrak{p}}} \underset{\Re}{\mathfrak{R}}\left\{\prod_{f \in \mathfrak{p}} \frac{\left(-\hbar_{f}\right)^{-b_{f}-1}}{b_{f} !} \mathcal{Z}^{\left(\mathfrak{a}\left(\Gamma_{\mathfrak{p}}\right)\right)}\left(\left(\hbar_{f}\right)_{f \in S_{\mathfrak{p}}},\left(\alpha_{v}^{\prime}\right)_{f \in \mathfrak{p}},\left(\alpha_{i_{f}}\right)_{f \in S_{\mathfrak{p}}^{*}}, q\right)\right\} .
\end{aligned}
$$

The sum on the left-hand side above is taken over all equivalence classes of connected $[n]$-valued $N$-marked genus $g$ weighted graphs $\Gamma$ as in (3.21) determining a fixed element (4.18) of $\mathcal{P}_{g, N}^{(m)}$ with 
$m=\left|\overline{\mathrm{Fl}}_{v}^{*}(\Gamma)\right|$. The analogue of (4.14) is now

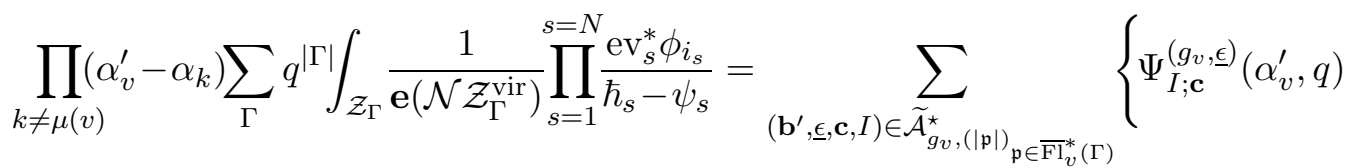

$$
\begin{aligned}
& \times \prod_{\mathfrak{p} \in \overline{\mathrm{F}}_{v}^{*}(\Gamma)} \frac{1}{|\mathfrak{p}| !} \underset{\left(\hbar_{f}=0\right)_{f \in \mathfrak{p}}}{\mathfrak{R}}\left\{\prod_{f \in \mathfrak{p}} \frac{e^{-\frac{\zeta\left(\alpha_{v}^{\prime}, q\right)}{\hbar_{f}}}}{b_{f}^{\prime} ! \hbar_{f}^{b_{f}^{\prime}+\epsilon_{f}+1}} \mathcal{Z}^{\left(\mathfrak{a}\left(\Gamma_{\mathfrak{p}}\right)\right)}\left(\left(\hbar_{f}\right)_{f \in S_{\mathfrak{p}}},\left(\alpha_{v}^{\prime}\right)_{f \in \mathfrak{p}},\left(\alpha_{i_{f}}\right)_{f \in S_{\mathfrak{p}}^{*}}, q\right)\right\},
\end{aligned}
$$

with $\widetilde{\mathcal{A}}_{g_{v},(|\mathfrak{p}|)_{\mathfrak{p} \in \overline{\mathrm{Fi}}}^{*}(\Gamma)}$ as in (2.30).

Suppose $\mathfrak{p} \in \overline{\mathrm{Fl}}_{v}^{*}(\Gamma)$ and $2 \mathfrak{a}\left(\Gamma_{\mathfrak{p}}\right)+\left|S_{\mathfrak{p}}\right| \geq 3$. Since $3 \mathfrak{a}\left(\Gamma_{\mathfrak{p}}\right)+\left|S_{\mathfrak{p}}\right|<3 g+N$, by induction Theorem B implies that

$$
\begin{aligned}
\mathcal{Z}^{\left(\mathfrak{a}\left(\Gamma_{\mathfrak{p}}\right)\right)}\left(\left(\hbar_{f}\right)_{f \in S_{\mathfrak{p}}},\left(\mathbf{x}_{f}\right)_{f \in S_{\mathfrak{p}}}, q\right) & \\
= & \sum_{\mathbf{p} \in\left\lfloor n \rrbracket^{S_{\mathfrak{p}}}\right.} \sum_{\mathbf{b} \in(\mathbb{Z} \geq 0)} \sum_{d=0}^{\infty} \mathcal{C}_{\mathfrak{a}\left(\Gamma_{\mathfrak{p}}\right) ; \mathbf{p}, \mathbf{b}}^{(d)} q^{d} \prod_{f \in S_{\mathfrak{p}}} \hbar_{f}^{-b_{f}-1} \mathcal{Z}_{p_{f}}\left(\hbar_{f}, \mathbf{x}_{f}, q\right)
\end{aligned}
$$

with $\mathcal{C}_{\mathfrak{a}\left(\Gamma_{\mathfrak{p}}\right) ; \mathbf{p}, \mathbf{b}}^{(d)} \in \mathbb{Q}[\alpha]$ satisfying $(\underline{3.12})$. We set $\mathcal{C}_{\mathfrak{a}\left(\Gamma_{\mathfrak{p}}\right) ; \mathbf{p}, \mathbf{b}}^{(d)}=0$ if $2 \mathfrak{a}\left(\Gamma_{\mathfrak{p}}\right)+\left|S_{\mathfrak{p}}\right| \geq 3$ and $b_{f}^{\prime}<0$ for some $f \in S_{\mathfrak{p}}$. Along with (3.33), the last equation implies that

$$
\begin{aligned}
\underset{\substack{\left.\hbar_{f}=0\right)_{f \in \mathfrak{p}} \\
\mathfrak{R}}}{ }\left\{\prod_{f \in \mathfrak{p}} \frac{e^{-\frac{\zeta\left(\alpha_{v}^{\prime}, q\right)}{\hbar_{f}}}}{\hbar_{f}^{b_{f}^{\prime}+\epsilon_{f}+1}} \mathcal{Z}^{\left(\mathfrak{a}\left(\Gamma_{\mathfrak{p}}\right)\right)}\left(\left(\hbar_{f}\right)_{f \in S_{\mathfrak{p}}},\left(\alpha_{v}^{\prime}\right)_{f \in \mathfrak{p}},\left(\alpha_{i_{f}}\right)_{f \in S_{\mathfrak{p}}^{*}}, q\right)\right\} \\
=\sum_{\substack{\mathbf{p} \in\left\lfloor n \rrbracket_{\mathfrak{p}}^{S_{\mathfrak{p}}^{*}} \\
\mathbf{b} \in(\mathbb{Z} \geq 0)^{S_{\mathfrak{p}}^{*}}\right.}}\left\{\prod_{f \in S_{\mathfrak{p}}^{*}} \hbar_{f}^{-b_{f}-1} \mathcal{Z}_{p_{f}}\left(\hbar_{f}, \alpha_{i_{f}}, q\right)\right. \\
\left.\times \sum_{\substack{\mathbf{p}^{\prime} \in \llbracket n \rrbracket^{S_{\mathfrak{p}}^{\prime}} \\
\mathbf{b}^{\prime \prime} \in \mathbb{Z}^{S_{\mathfrak{p}}^{\prime}}}} \sum_{d=0}^{\infty} \mathcal{C}_{\mathfrak{a}\left(\Gamma_{\mathfrak{p}}\right) ; \mathbf{p p}^{\prime}, \mathbf{b b}^{\prime \prime}}^{(d)} q^{d} \prod_{f \in \mathfrak{p}} \Psi_{p_{f}^{\prime} ; b_{f}^{\prime}+\epsilon_{f}+1+b_{f}^{\prime \prime}}\left(\alpha_{v}^{\prime}, q\right)\right\}
\end{aligned}
$$

if $2 \mathfrak{a}\left(\Gamma_{\mathfrak{p}}\right)+\left|S_{\mathfrak{p}}\right| \geq 3$. By Lemma 3.2 and Corollary 3.3, (4.23) with

$$
\mathcal{C}_{0 ;\left(p_{+}, p_{-}\right),\left(b_{+}, b_{-}\right)}^{(d)}= \begin{cases}(-1)^{b_{+}} \llbracket \mathcal{C}_{p_{-} p_{+}}(q) \rrbracket_{q ; d}, & \text { if } b_{+} \geq 0, b_{-}+b_{+}=-1 \\ 0, & \text { otherwise }\end{cases}
$$

holds if $\mathfrak{a}\left(\Gamma_{\mathfrak{p}}\right)=0$ and $\left|S_{\mathfrak{p}}\right|=2$.

We note that

$$
\prod_{\mathfrak{p} \in \overline{\mathrm{Fl}}_{v}^{*}(\Gamma)} \prod_{f \in S_{\mathfrak{p}}^{*}} \hbar_{f}^{-b_{f}-1} \mathcal{Z}_{p_{f}}\left(\hbar_{f}, \alpha_{i_{f}}, q\right)=\underline{\hbar}^{-\mathbf{b}} \mathcal{Z}_{\mathbf{p}}\left(\underline{\hbar}, \alpha_{i_{1} \ldots i_{N}}, q\right) \quad \forall \mathbf{p} \in \llbracket n \rrbracket^{N}, \mathbf{b} \in\left(\mathbb{Z}^{\geq 0}\right)^{N} .
$$

We now divide both sides of (4.22) by the first factor on the left-hand side, plug in (4.23), and sum up over all possibilities for $\mu(v) \in[n]$ using the Residue Theorem on $\mathbb{P}^{1}$, as after (4.14). Summing 
up the result over all possibilities for (4.18), we obtain a recursion for the coefficients $\mathcal{C}_{g ; \mathbf{p}, \mathbf{b}}^{(d)}$ in Theorem B;

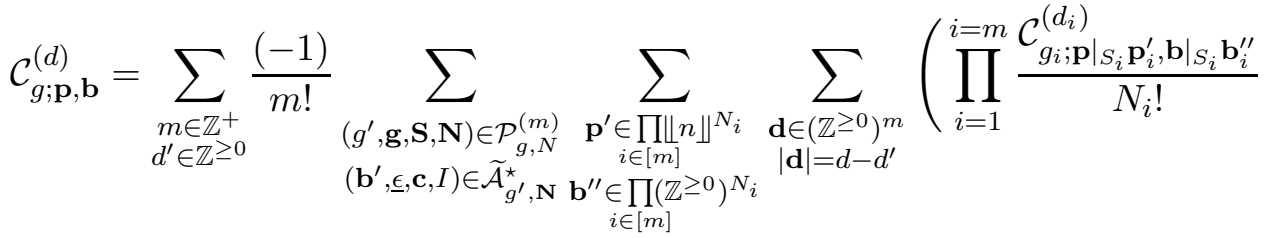

$$
\begin{aligned}
& \left.\underset{\mathbf{x}=0, \infty}{\mathfrak{R}} \llbracket \frac{\Psi_{I ; \mathbf{c}}^{\left(g^{\prime}, \underline{\epsilon}\right)}(\mathbf{x}, q)}{\prod_{k=1}^{k=n}\left(\mathbf{x}-\alpha_{k}\right)} \prod_{i=1}^{i=m} \prod_{f \in\left[N_{i}\right]} \frac{\Psi_{p_{i ; f}^{\prime} ; b_{i ; f}^{\prime}+\epsilon_{i ; f}+1+b_{i ; f}^{\prime \prime}(\mathbf{x}, q)}}{b_{i ; f}^{\prime} !} \rrbracket_{q ; d^{\prime}}\right) .
\end{aligned}
$$

It remains to show that (3.12) with the summation over $t \in \mathbb{Z}$ instead of $\mathbb{Z} \geq 0$ holds for some $\mathrm{c}_{g ; \mathbf{p}, \mathbf{b}}^{(d, t)} \in \mathbb{Q}$ such that $\mathrm{c}_{g ; \mathbf{p}, \mathbf{b}}^{(d, t)}$ with $t \geq 0$ satisfy (2.33).

By (4.25) and (4.16),

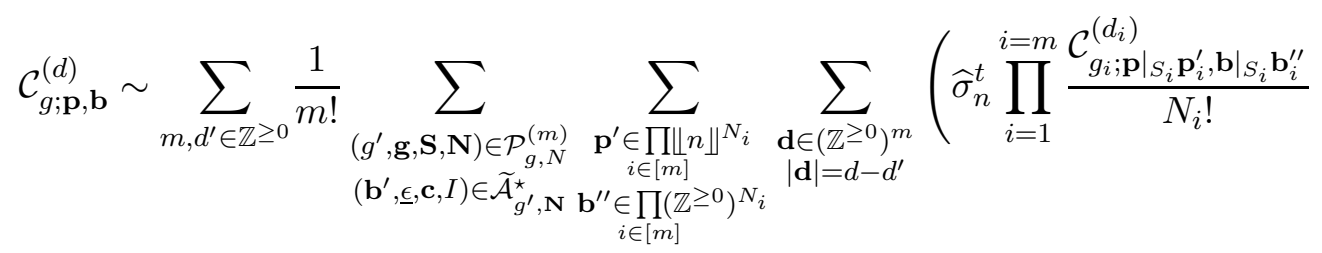

$$
\begin{aligned}
& \left.\times \llbracket \Phi_{I ; \mathbf{c}}^{\left(g^{\prime}, \underline{\epsilon}\right)}(q) \prod_{i=1}^{i=m} \prod_{f \in\left[N_{i}\right]} \frac{\Phi_{p_{i ; f}^{\prime} ; b_{i ; f}^{\prime}+\epsilon_{i ; f}+1+b_{i ; f}^{\prime \prime}(q)}}{b_{i ; f}^{\prime} ! \Phi_{0}(q)} \|_{q ; d^{\prime}}\right),
\end{aligned}
$$

with $t \in \mathbb{Z}$ defined by

$$
\left|\mathbf{p}^{\prime}\right|-\left|\mathbf{b}^{\prime \prime}\right|=(n-4)\left(1-g^{\prime}\right)+2|\mathbf{N}|+n\left(d^{\prime}+t\right) \quad \Longleftrightarrow \quad\left(\mathbf{p}^{\prime}, \mathbf{b}^{\prime \prime}\right) \in \mathcal{S}_{g^{\prime}, \mathbf{N}}\left(d^{\prime}, t\right) ;
$$

if an integer $t$ satisfying the above condition does not exist, we define $\widehat{\sigma}_{n}^{t}$ to be 0 .

We now confirm (3.12) by induction on $3 g+N$. For $(g, N)=(0,2)$, (3.12) holds by (4.24), (3.34), and (2.32). Suppose $3 g+N \geq 0$ and (3.12) holds for smaller values of this sum. If $\left(g^{\prime}, \mathbf{g}, \mathbf{S}, \mathbf{N}\right)$ is an element of $\mathcal{P}_{g, N}^{(m)}$ for some $m \in \mathbb{Z}^{+}$, then

$$
3 g_{i}+\left|S_{i}\right|+N_{i}<3 g+N \quad \forall i \in[m] .
$$

Thus, (4.26) with the roles of $\mathbf{b}^{\prime}$ and $\mathbf{b}^{\prime \prime}$ interchanged and (3.12) with $(g, N)$ replaced by $\left(g_{i},\left|S_{i}\right|+N_{i}\right)$ imply that

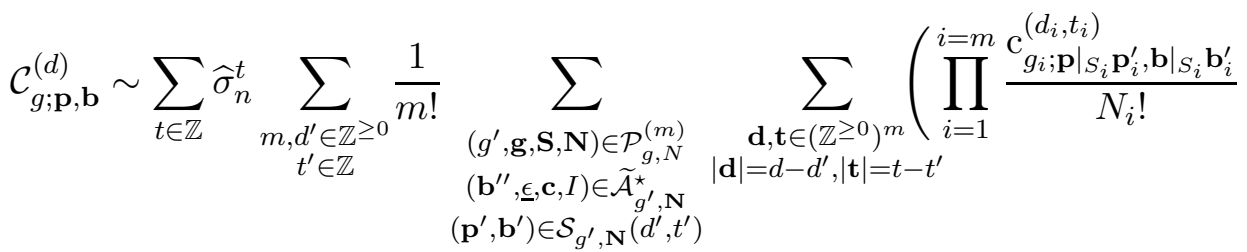

$$
\begin{aligned}
& \left.\times \llbracket \Phi_{I ; \mathbf{c}}^{\left(g^{\prime}, \underline{\epsilon}\right)}(q) \prod_{i=1}^{i=m} \prod_{f \in\left[N_{i}\right]} \frac{\Phi_{p_{i ; f}^{\prime} ; b_{i ; f}^{\prime \prime}+\epsilon_{i ; f}+1+b_{i ; f}^{\prime}(q)}}{b_{i ; f}^{\prime \prime} ! \Phi_{0}(q)} \rrbracket_{q ; d^{\prime}}\right) .
\end{aligned}
$$

By (2.33), this expression reduces to (3.12). 


\section{$5 \quad$ Key combinatorial identities}

We now establish the key combinatorial statements, Propositions 2.1 and 5.8, which characterize the Hodge integrals (2.5) and sums of residues of well-behaved generating series of rational functions as in Proposition 3.4, respectively. Their proofs make use of the basic identities of Lemma 5.1 below. Lemma 5.2 is used in the proof of Theorem 1 .

Lemma 5.1 ([22, Lemma B.1]). The following identities hold:

$$
\begin{aligned}
& \sum_{\substack{\mathbf{b}^{\prime} \in(\mathbb{Z} \geq 0)^{m} \\
\left|\mathbf{b}^{\prime}\right|=b^{\prime}}} \prod_{k=1}^{k=m}\left(\begin{array}{l}
b_{k} \\
b_{k}^{\prime}
\end{array}\right)=\left(\begin{array}{c}
b_{1}+\ldots+b_{m} \\
b^{\prime}
\end{array}\right) \quad \forall m \in \mathbb{Z}^{+}, b_{1}, \ldots, b_{m}, b^{\prime} \in \mathbb{Z}^{\geq 0}, \\
& \sum_{b=0}^{\infty}(-1)^{b}\left(\begin{array}{l}
p \\
b
\end{array}\right)\left(\begin{array}{c}
B+b \\
s
\end{array}\right)=(-1)^{p}\left(\begin{array}{c}
B \\
s-p
\end{array}\right) \quad \forall B, p, s \in \mathbb{Z}^{\geq 0}, \\
& \sum_{p=0}^{\infty}(-1)^{p}\left(\begin{array}{c}
m+p \\
p
\end{array}\right) \Psi^{p}=\frac{1}{(1+\Psi)^{m+1}} \quad \forall m \in \mathbb{Z}^{\geq 0} .
\end{aligned}
$$

Lemma 5.2. Let $g, N \in \mathbb{Z} \geq 0$ with $2 g+N \geq 3$ and $\Gamma \in \mathcal{A}_{g, N}$ be a connected trivalent $N$-marked genus g graph as in (2.23). Then,

$$
\begin{aligned}
\sum_{\mathbf{c} \in((\mathbb{Z} \geq 0) \infty)^{\mathrm{Ver}}} 2^{-\|\mathbf{c}\|} \prod_{v \in \operatorname{Ver}}\left(\frac{\left(3\left(g_{v}-1\right)+\left|\overline{\mathrm{Fl}}_{v}(\Gamma)\right|+\left|\mathbf{c}_{v}\right|\right) !}{\left(3\left(g_{v}-1\right)+\left|\overline{\mathrm{Fl}}_{v}(\Gamma)\right|\right) !\left|\mathbf{c}_{v}\right| !}\left(\begin{array}{c}
\left|\mathbf{c}_{v}\right| \\
\mathbf{c}_{v}
\end{array}\right) \prod_{r=1}^{\infty}\left(\frac{1}{r+1}\right)^{c_{v ; r}}\right) \\
=(2(1-\ln 2))^{-\left(3 g+N-2-g_{\Gamma}\right)} .
\end{aligned}
$$

Proof. For each $v \in$ Ver, let $m_{v}=3\left(g_{v}-1\right)+\left|\overline{\mathrm{Fl}}_{v}(\Gamma)\right|$ as before. Since $\Gamma$ is trivalent, $m_{v} \geq 0$. Since $|2 \ln 2-1|<1$, the Binomial Theorem gives

$$
\begin{aligned}
\sum_{\mathbf{c}_{v} \in(\mathbb{Z} \geq 0)^{\infty}} 2^{-\left\|\mathbf{c}_{v}\right\|} \frac{\left(m_{v}+\left|\mathbf{c}_{v}\right|\right) !}{m_{v} !\left|\mathbf{c}_{v}\right| !}\left(\begin{array}{c}
\left|\mathbf{c}_{v}\right| \\
\mathbf{c}_{v}
\end{array}\right) \prod_{r=1}^{\infty}\left(\frac{1}{r+1}\right)^{c_{v ; r}}=\sum_{c=0}^{\infty} \frac{\left(m_{v}+c\right) !}{m_{v} ! c !}\left(\sum_{r=1}^{\infty} \frac{2^{-r}}{r+1}\right)^{c} \\
=\sum_{c=0}^{\infty} \frac{\left(m_{v}+c\right) !}{m_{v} ! c !}(2 \ln 2-1)^{c}=(2(1-\ln 2))^{-\left(m_{v}+1\right)} .
\end{aligned}
$$

Combining this with the second equality in (2.25) and with the first equality in (2.24), we obtain the claim.

\subsection{Proof of Proposition 2.1}

Let $m \in \mathbb{Z}^{\geq 0}$. For a tuple $\mathbf{b} \equiv\left(b_{k}\right)_{k \in[m]}$ in $\left(\mathbb{Z}^{\geq 0}\right)^{m}$, we define

$$
\left(\begin{array}{c}
|\mathbf{b}| \\
\mathbf{b}
\end{array}\right)=\left(\begin{array}{c}
|\mathbf{b}| \\
b_{1}, \ldots, b_{m}
\end{array}\right) \equiv \frac{|\mathbf{b}| !}{b_{1} ! \ldots b_{m} !}
$$

We extend this definition to arbitrary tuples $\mathbf{b}$ in $\mathbb{Z}^{m}$ by setting

$$
\left(\begin{array}{c}
|\mathbf{b}| \\
\mathbf{b}
\end{array}\right)=0 \quad \text { if } \quad b_{k}<0 \text { for some } k \in[m] .
$$


For $g, m \in \mathbb{Z}^{\geq 0}$ with $2 g+m \geq 3$ and $I \in\left(\mathbb{Z}^{\geq 0}\right)^{g}$, we extend the definition in (2.5) to arbitrary tuples $\mathbf{b}$ in $\left(\mathbb{Z}^{\geq 0}\right)^{m}$ by setting

$$
\left\langle\left\langle\lambda_{g ; I} ; \tau_{\mathbf{b}}\right\rangle\right\rangle,\left\langle\left\langle\lambda_{g ; I} ; \widetilde{\tau}_{\mathbf{b}}\right\rangle\right\rangle=0 \quad \text { if } \quad b_{k}<0 \text { for some } k \in[m] .
$$

Lemma 5.3. Let $g \in \mathbb{Z}^{\geq 0}$ and $I \in\left(\mathbb{Z}^{\geq 0}\right)^{g}$. There exists a collection

$$
C_{I ; \underline{\epsilon}}^{(g)} \in \mathbb{Q} \quad \text { with } \quad \underline{\epsilon} \in\left(\mathbb{Z}^{\geq 0}\right)^{m}, m \in \mathbb{Z}^{\geq 0}, 2 g+m \geq 3,
$$

which is invariant under the permutations of the components of $\underline{\epsilon}$ such that

$$
\left\langle\left\langle\lambda_{g ; I} ; \tau_{\mathbf{b}}\right\rangle\right\rangle=\sum_{\substack{\epsilon \in(\mathbb{Z} \geq 0)^{m} \\
|\underline{\epsilon}| \leq \mu_{g}(I)+m}} C_{I ; \underline{\epsilon})}^{(g)}\left(\begin{array}{c}
|\mathbf{b}|-|\underline{\epsilon}| \\
\mathbf{b}-\underline{\epsilon}
\end{array}\right)
$$

for all $m \in \mathbb{Z}^{\geq 0}$ and $\mathbf{b} \in \mathbb{Z}^{m}$ with $2 g+m \geq 3$ and $|\mathbf{b}| \geq \mu_{g}(I)+m$,

$$
C_{I ; \underline{0} 0}^{(g)}=C_{I ; \underline{\epsilon}}^{(g)}, \quad C_{I ; \underline{1} 1}^{(g)}=(|\underline{\epsilon}|+\|I\|-g) C_{I ; \underline{\epsilon}}^{(g)} \quad \text { if }(g, I) \neq(1,(0)) \text { or }|\underline{\epsilon}|>1
$$

and $C_{I ; \underline{\epsilon}}^{(g)}=0$ if $|\underline{\epsilon}|_{S}>\mu_{g}(I)+|S|$ for some subset $S \subset[m]$ with $2 g+|S| \geq 3$. If $(g, I)=(1,(0))$, then the numbers in (5.1) can be chosen so that in addition

$$
C_{(0) ;(0)}^{(1)}=0, \quad C_{(0) ;(1,1)}^{(1)}=-C_{(0) ;(1)}^{(1)} .
$$

Proof. We set the numbers in (5.1) to be 0 whenever $|\underline{\epsilon}|_{S}>\mu_{g}(I)+|S|$ for some subset $S \subset[m]$ with $2 g+|S| \geq 3$. Thus,

$$
\begin{gathered}
C_{I ; \underline{\epsilon}}^{(g)}=0 \text { if }\|I\|>\max (g, 3 g-3), \\
C_{() ; \underline{\epsilon}}^{(0)}, C_{(1) ; \underline{\epsilon}}^{(1)}=0 \quad \text { if } \underline{\epsilon} \in \mathbb{Z}^{m}-\left\{0^{m}\right\}, \quad C_{(0) ; \underline{\epsilon}}^{(1)}=0 \text { if } \underline{\epsilon} \in \mathbb{Z}^{m}-\{0,1\}^{m} .
\end{gathered}
$$

With $\mathbf{0}_{m} \in \mathbb{Z}^{m}$ denoting the zero vector, we also define

$$
\begin{aligned}
C_{() ; \mathbf{0}_{m}}^{(0)} & =C_{() ; \mathbf{0}_{3}}^{(0)} \forall m>3, \\
C_{(1) ; \mathbf{0}_{m}}^{(1)} & =C_{(1) ; \mathbf{0}_{1}}^{(1)} \forall m>1,
\end{aligned} \quad C_{(0) ; \underline{\epsilon}}^{(1)}= \begin{cases}0, & \text { if } \underline{\epsilon}=\mathbf{0}_{m} ; \\
-(|\underline{\epsilon}|-2) ! C_{(0) ;(1)}^{(1)}, & \text { if } \underline{\epsilon} \in\{0,1\}^{m},|\epsilon| \geq 2 .\end{cases}
$$

The coefficients defined in this way are invariant under the permutations of the components of $\underline{\epsilon}$ and satisfy (5.3), the vanishing condition after (5.3), and (5.4) if $(g, I)=(1,(0))$.

Let $g \geq 2$. We set

$$
C_{I ; \underline{\epsilon}}^{(g)}=0 \quad \text { if } \quad|\underline{\epsilon}|<\mu_{g}(I)+m \text { and } \epsilon_{k} \geq 2 \forall k \in[m] .
$$

Suppose $m \in \mathbb{Z}^{+}$and the numbers $C_{I ; \underline{\epsilon}}^{(g)} \in \mathbb{Q}$ with $\underline{\epsilon} \in\left(\mathbb{Z}^{\geq 0}\right)^{m-1}$ are invariant under the permutations of the components of $\underline{\epsilon}$. If $m \geq 2$, assume in addition that

$$
C_{I ; \underline{1} 1}^{(g)}=(|\underline{\epsilon}|+\|I\|-g) C_{I ; \underline{0} 0}^{(g)} \quad \forall \underline{\epsilon} \in\left(\mathbb{Z}^{\geq 0}\right)^{m-2} .
$$

The conditions (5.3) and the symmetry requirement then determine the numbers

$$
C_{I ; \underline{\epsilon}}^{(g)} \in \mathbb{Q} \quad \text { with } \quad \underline{\epsilon} \equiv\left(\epsilon_{1}, \ldots, \epsilon_{m}\right) \in\left(\mathbb{Z}^{\geq 0}\right)^{m} \text { s.t. } \epsilon_{k}<2 \text { for some } k \in[m] .
$$


If $m \geq 2$, the condition (5.7) holds if the numbers $C_{I ; \underline{\epsilon}}^{(g)} \in \mathbb{Q}$ with $\underline{\epsilon} \in\left(\mathbb{Z}^{\geq 0}\right)^{m-1}$ satisfy (5.3). Thus, the numbers (5.1) invariant under the permutations of the components of $\underline{\epsilon}$ and satisfying (5.3), the vanishing condition after (5.3), and the additional condition (5.6) are determined by the numbers

$$
C_{I ; \underline{\epsilon}}^{(g)} \in \mathbb{Q} \quad \text { with } \underline{\epsilon} \equiv\left(\epsilon_{1}, \ldots, \epsilon_{m}\right) \in\left(\mathbb{Z}^{\geq 0}\right)^{m}, m \in \mathbb{Z}^{\geq 0}, \quad \epsilon_{k} \geq 2 \forall k \in[m],|\underline{\epsilon}|=\mu_{g}(I)+m
$$

invariant under the permutations of the components of $\underline{\epsilon}$.

Let $g \in \mathbb{Z}^{\geq 0}$ be arbitrary. For $m \in \mathbb{Z}^{\geq 0}$ and $i \in[m]$, denote by $e_{i} \in \mathbb{Z}^{m}$ the $i$-th standard coordinate vector. For all $\mathbf{b} \in \mathbb{Z}^{m}-\{0\}$,

$$
\left(\begin{array}{c}
|\mathbf{b}| \\
\mathbf{b}
\end{array}\right)=\sum_{i=1}^{i=m}\left(\begin{array}{c}
|\mathbf{b}|-1 \\
\mathbf{b}-e_{i}
\end{array}\right)
$$

For $m \in \mathbb{Z}^{\geq 0}$ and $\mathbf{b} \in\left(\mathbb{Z}^{\geq 0}\right)^{m}$ with $2 g+m \geq 3$ and $|\mathbf{b}|=\mu_{g}(I)+m$, let

$$
\left\langle\langle\mathbf{b}\rangle_{g ; I}=\sum_{\substack{\epsilon \in(\mathbb{Z} \geq 0)^{m} \\
|\underline{\epsilon}| \leq \mu_{g}(I)+m}} C_{I ; \underline{\epsilon}}^{(g)}\left(\begin{array}{c}
|\mathbf{b}|-|\underline{\epsilon}| \\
\mathbf{b}-\underline{\epsilon}
\end{array}\right) .\right.
$$

By the first relation in (5.3), the vanishing condition after (5.3), and (5.10),

$$
\langle\langle\mathbf{b} 0\rangle\rangle_{g ; I}=\sum_{i=1}^{i=m}\left\langle\left\langle\mathbf{b}-e_{i}\right\rangle\right\rangle_{g ; I}
$$

for all $m \in \mathbb{Z}^{\geq 0}$ and $\mathbf{b} \in\left(\mathbb{Z}^{\geq 0}\right)^{m}$ with $2 g+m \geq 3$ and $|\mathbf{b}|=\mu_{g}(I)+m+1$. By (5.3) and the vanishing condition after (5.3),

$$
\langle\langle\mathbf{b} 1\rangle\rangle_{g ; I}=(|\mathbf{b}|+1-g+\|I\|)\langle\langle\mathbf{b}\rangle\rangle_{g ; I}
$$

for all $m \in \mathbb{Z}^{\geq 0}$ and $\mathbf{b} \in\left(\mathbb{Z}^{\geq 0}\right)^{m}$ with $2 g+m \geq 3$ and $|\mathbf{b}|=\mu_{g}(I)+m$; if $(g, I)=(1,(0))$, we also need to use (5.4) and (5.10) to obtain (5.12).

Let $m \in \mathbb{Z}^{\geq 0}$ with $2 g+m \geq 3$. By the string and dilaton equations [11, Section 26.3],

$$
\begin{aligned}
& \left\langle\left\langle\lambda_{g ; I} ; \tau_{\mathbf{b} 0}\right\rangle\right\rangle=\sum_{i=1}^{i=m}\left\langle\left\langle\lambda_{g ; I} ; \tau_{\mathbf{b}-e_{i}}\right\rangle\right\rangle \quad \text { and } \\
& \left\langle\left\langle\lambda_{g ; I} ; \tau_{\mathbf{b} 1}\right\rangle\right\rangle=(|\mathbf{b}|+1-g+\|I\|)\left\langle\left\langle\lambda_{g ; I} ; \tau_{\mathbf{b}}\right\rangle\right\rangle,
\end{aligned}
$$

respectively. By the first case in (2.4) and the first identity in (5.13),

$$
\left\langle\left\langle\lambda_{g ; I} ; \tau_{\mathbf{b}}\right\rangle\right\rangle=\left\langle\left\langle\lambda_{g ; I} ; \tau_{\mathbf{b} 0}\right\rangle\right\rangle=\sum_{i=1}^{i=m}\left\langle\left\langle\lambda_{g ; I} ; \tau_{\mathbf{b}-e_{i}}\right\rangle\right\rangle \quad \text { if }|\mathbf{b}|>\mu_{g}(I)+m .
$$

Along with (5.11), this implies that (5.2) for all $\mathbf{b} \in\left(\mathbb{Z}^{\geq 0}\right)^{m}$ and $|\mathbf{b}| \geq \mu_{g}(I)+m$ is equivalent to

$$
\left\langle\left\langle\lambda_{g ; I} ; \tau_{\mathbf{b}}\right\rangle\right\rangle=\langle\langle\mathbf{b}\rangle\rangle_{g ; I} \quad \forall \mathbf{b} \in\left(\mathbb{Z}^{\geq 0}\right)^{m} \text { s.t. }|\mathbf{b}|=\mu_{g}(I)+m .
$$

By the symmetry of the numbers on the two sides of (5.14) and (5.11) with $2 g+m \geq 3$ is in turn equivalent to (5.14) for all $m \in \mathbb{Z}^{\geq 0}$ and $\mathbf{b} \in\left(\mathbb{Z}^{\geq 0}\right)^{m}$ with either $2 g+m=3$ 
or $2 g+m>3$ and $b_{k} \geq 2$ for all $k \in[m]$.

For $g=0,1$, (5.2) thus reduces to

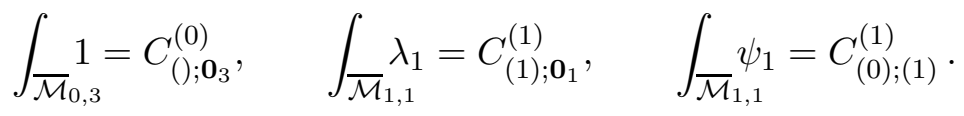

For $g \geq 2$, (5.2) reduces to

$$
\int_{\overline{\mathcal{M}}_{g, m}} \lambda_{g ; I} \prod_{k=1}^{m} \psi_{k}^{b_{k}}=\sum_{\substack{\epsilon \in \in(\mathbb{Z} \geq 0)^{m} \\
|\underline{\epsilon}| \leq \mu_{g}(I)+m}} C_{I ; \underline{\epsilon}}^{(g)}\left(\begin{array}{c}
|\mathbf{b}|-|\underline{\epsilon}| \\
\mathbf{b}-\underline{\epsilon}
\end{array}\right)
$$

for all $m \in \mathbb{Z}^{\geq 0}$ and $\mathbf{b} \equiv\left(b_{k}\right)_{k \in[m]} \in\left(\mathbb{Z}^{\geq 0}\right)^{m}$ with $|\mathbf{b}|=\mu_{g}(I)+m$ and $b_{k} \geq 2$ for all $k \in[m]$. We take $C_{I ;()}^{(g)}$ to be the $m=0$ number on the left-hand side of (5.16); it vanishes unless $\mu_{g}(I)=0$.

Suppose $g \geq 2, \mu_{g}(I) \geq 0, m^{*} \in \mathbb{Z}^{+}$, and we have constructed the numbers (5.1) with the required properties for all $m<m^{*}$. The only numbers (5.1) for $m=m^{*}$ that remain to be determined are the numbers $C_{I ; \underline{\epsilon}}^{(g)}$ in (5.9) with $m=m^{*}$. The only one of these numbers appearing in the equation (5.16) corresponding to $\mathbf{b} \in\left(\mathbb{Z}^{\geq 0}\right)^{m^{*}}$ with $|\mathbf{b}|=\mu_{g}(I)+m^{*}$ with a nonzero coefficient is the number indexed by $\underline{\epsilon}=\mathbf{b}$. Thus, the equations (5.16) with $|\mathbf{b}|=\mu_{g}(I)+m^{*}$ determine the numbers $C_{I ; \underline{\epsilon}}^{(g)}$ in (5.9) with $m=m^{*}$. By the invariance of the sides two of (5.16) under the permutations of the components of $\mathbf{b}$, the numbers $C_{I ; \underline{\epsilon}}^{(g)}$ in (5.9) determined by these equations are invariant under the permutations of the components of $\underline{\epsilon}$. This establishes the existence of the numbers $C_{I ; \underline{\epsilon}}^{(g)}$ in (5.1) satisfying all requirement of the lemma.

Lemma 5.4. Let $g \in \mathbb{Z}^{\geq 0}$ and $I \in\left(\mathbb{Z}^{\geq 0}\right)^{g}$. With $C_{I ; \underline{\epsilon}}^{(g)}$ as in Lemma 5.3,

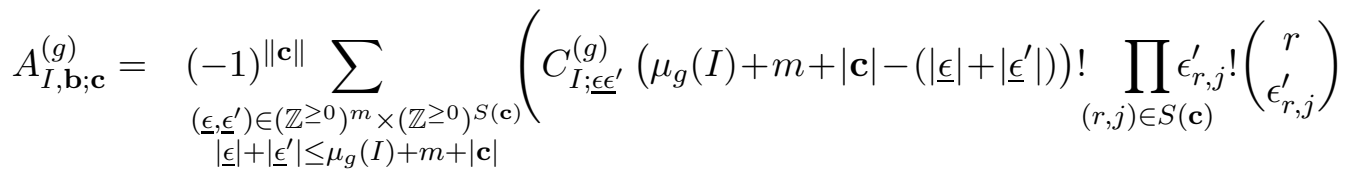

$$
\begin{aligned}
& \left.\times\left(\begin{array}{c}
|\mathbf{b}|-|\underline{\epsilon}| \\
\mu_{g}(I)+m+|\mathbf{c}|-\|\mathbf{c}\|-|\underline{\epsilon}|
\end{array}\right) \prod_{k=1}^{m} \epsilon_{k} !\left(\begin{array}{l}
b_{k} \\
\epsilon_{k}
\end{array}\right)\right) .
\end{aligned}
$$

for all $m \in \mathbb{Z}^{\geq 0}$ with $2 g+m \geq 3, \mathbf{b} \equiv\left(b_{k}\right)_{k \in[m]} \in\left(\mathbb{Z}^{\geq 0}\right)^{m}$, and $\mathbf{c} \in\left(\mathbb{Z}^{\geq 0}\right)^{\infty}$.

Proof. By (5.2) and the second case in (2.4),

$$
\begin{aligned}
A_{I, \mathbf{b} ; \mathbf{c}}^{(g)}= & \sum_{\substack{\mathbf{b}^{\prime} \in\left(\mathbb{Z}^{\geq 0}\right)^{S(\mathbf{c})} \\
\left(\epsilon, \epsilon^{\prime}\right) \in\left(\mathbb{Z}^{\geq 0}\right)^{m} \times(\mathbb{Z} \geq 0) S(\mathbf{c}) \\
|\underline{\epsilon}|+\left|\underline{\epsilon}^{\prime}\right| \leq \mu_{g}(I)+m+\mathbf{c}|\leq| \mathbf{b}|+| \mathbf{b}^{\prime} \mid}}\left(\frac{(-1)^{\left|\mathbf{b}^{\prime}\right|} C_{I ; \underline{\epsilon^{\prime}}}^{(g)}\left(|\mathbf{b}|+\left|\mathbf{b}^{\prime}\right|-\left(|\underline{\epsilon}|+\left|\underline{\epsilon}^{\prime}\right|\right)\right) !}{\left(|\mathbf{b}|+\left|\mathbf{b}^{\prime}\right|-\left(\mu_{g}(I)+m+|\mathbf{c}|\right)\right) !}\right. \\
& \left.\times \prod_{k=1}^{m} \epsilon_{k} !\left(\begin{array}{c}
b_{k} \\
\epsilon_{k}
\end{array}\right) \prod_{(r, j) \in S(\mathbf{c})} \frac{r !}{\left(r-\epsilon_{r, j}^{\prime}\right) !}\left(\begin{array}{c}
r-\epsilon_{r, j}^{\prime} \\
b_{r, j}^{\prime}-\epsilon_{r, j}^{\prime}
\end{array}\right)\right) .
\end{aligned}
$$


If $\mathbf{c}=\mathbf{0}$ and $|\mathbf{b}| \geq \mu_{g}(I)+m$, this expression becomes

$$
A_{I, \mathbf{b} ; \mathbf{0}}^{(g)}=\sum_{\substack{\epsilon \in(\mathbb{Z} \geq 0) m \\
|\epsilon| \leq \mu_{g}(I)+m}} \frac{C_{I ; \underline{\underline{\epsilon}}}^{(g)}(|\mathbf{b}|-|\underline{\epsilon}|) !}{\left(|\mathbf{b}|-\left(\mu_{g}(I)+m\right)\right) !} \prod_{k=1}^{m} \epsilon_{k} !\left(\begin{array}{c}
b_{k} \\
\epsilon_{k}
\end{array}\right) .
$$

If $\mathbf{c}=\mathbf{0}$ and $|\mathbf{b}|<\mu_{g}(I)+m$, the first sum vanishes. This establishes the claim in both $\mathbf{c}=\mathbf{0}$ cases.

Suppose $\mathbf{c} \neq \mathbf{0}$. By (5.17) and the first statement in Lemma 5.1,

$$
\begin{aligned}
A_{I, \mathbf{b} ; \mathbf{c}}^{(g)}= & \sum_{\substack{\left(\underline{\epsilon}, \underline{\epsilon}^{\prime}\right) \in\left(\mathbb{Z}^{\geq 0}\right)^{m} \times\left(\mathbb{Z}^{\geq 0}\right)^{S(\mathbf{c})} \\
|\underline{\epsilon}|+\left|\underline{\epsilon}^{\prime}\right| \leq \mu_{g}(I)+m+|\mathbf{c}|}}\left((-1)^{\left|\underline{\epsilon}^{\prime}\right|} C_{I ; \underline{\epsilon^{\prime}}}^{(g)}\left(\mu_{g}(I)+m+|\mathbf{c}|-\left(|\underline{\epsilon}|+\left|\underline{\epsilon}^{\prime}\right|\right)\right) ! \prod_{(r, j) \in S(\mathbf{c})} \epsilon_{r, j}^{\prime} !\left(\begin{array}{c}
r \\
\epsilon_{r, j}^{\prime}
\end{array}\right)\right. \\
& \left.\times \prod_{k=1}^{m} \epsilon_{k} !\left(\begin{array}{c}
b_{k} \\
\epsilon_{k}
\end{array}\right) \sum_{b^{\prime}=0}^{\infty}(-1)^{b^{\prime}}\left(\begin{array}{c}
|\mathbf{b}|+b^{\prime}-|\underline{\epsilon}| \\
\mu_{g}(I)+m+|\mathbf{c}|-|\epsilon|-\left|\epsilon^{\prime}\right|
\end{array}\right)\left(\begin{array}{c}
\|\mathbf{c}\|-\left|\underline{\epsilon}^{\prime}\right| \\
b^{\prime}
\end{array}\right)\right) .
\end{aligned}
$$

The claim now follows from the second statement in Lemma 5.1 .

Proof of Proposition [2.1. By Lemma 5.4 and the vanishing statement after (5.3), (2.6) holds with

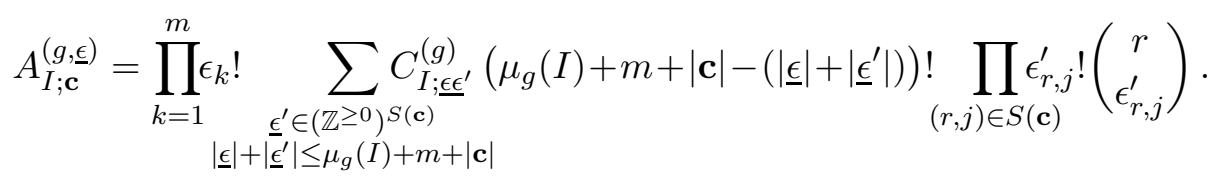

Since the coefficients $C_{I ; \epsilon \epsilon^{\prime}}^{(g)}$ provided by Lemma 5.3 are invariant under the permutations of the components of $\underline{\epsilon}$, so are the numbers (5.18).

By the vanishing statement in Lemma 5.3 ,

$$
\sum_{\substack{1 \leq i \leq m \\ \epsilon_{i} \geq 2}} \epsilon_{i} \leq 6 g+3 \quad \text { if } \underline{\epsilon} \in\left(\mathbb{Z}^{\geq 0}\right)^{m}, I \in\left(\mathbb{Z}^{\geq 0}\right)^{g}, 2 g+m \geq 3, C_{I ; \epsilon}^{(g)} \neq 0 .
$$

Along with (5.3), this implies that there exists $C_{g} \in \mathbb{R}$ such that

$$
\begin{aligned}
\prod_{i=1}^{i=m} \epsilon_{i} !\left|C_{I ; \underline{\epsilon}}^{(g)}\right|(3(g-1)+m-\|I\|-|\underline{\epsilon}|) ! & \leq C_{g}(|\underline{\epsilon}|+\|I\|) !(3(g-1)+m-\|I\|-|\underline{\epsilon}|) ! \\
& \leq C_{g}(3(g-1)+m) !
\end{aligned}
$$

for all $I \in\left(\mathbb{Z}^{\geq 0}\right)^{g}, \underline{\epsilon} \in\left(\mathbb{Z}^{\geq 0}\right)^{m}$, and $m \in \mathbb{Z}^{\geq 0}$ with $2 g+m \geq 3$. By (5.18), (5.19), and the first statement in Lemma 5.1,

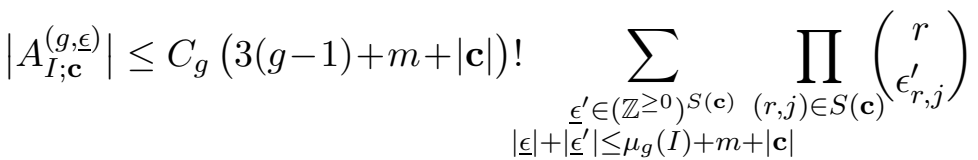

$$
\begin{aligned}
& \leq C_{g}(3(g-1)+m+|\mathbf{c}|) ! \sum_{\epsilon^{\prime}=0}^{\epsilon^{\prime}=\|\mathbf{c}\|}\left(\begin{array}{c}
\|\mathbf{c}\| \\
\epsilon^{\prime}
\end{array}\right)=C_{g} 2^{\|\mathbf{c}\|}(3(g-1)+m+|\mathbf{c}|) ! .
\end{aligned}
$$

This establishes (2.7). 
Corollary 5.5. Let $g \in \mathbb{Z}^{\geq 0}$ and $I \in\left(\mathbb{Z}^{\geq 0}\right)^{g}$. Then,

$$
A_{I, \mathbf{b} ;\left(c_{1}, c_{2}, \ldots\right)}^{(g)}=(-1)^{c_{1}} \frac{\left(2 g+m-3+c_{1}+c_{2}+\ldots\right) !}{\left(2 g+m-3+c_{2}+\ldots\right) !} A_{I, \mathbf{b} ;\left(0, c_{2}, \ldots\right)}^{(g)}
$$

for all $m \in \mathbb{Z}^{\geq 0}$ with $2 g+m \geq 3, \mathbf{b} \in\left(\mathbb{Z}^{\geq 0}\right)^{m}$, and $\mathbf{c} \equiv\left(c_{r}\right)_{r \in \mathbb{Z}^{+}} \in\left(\mathbb{Z}^{\geq 0}\right)^{\infty}$.

Proof. Let $A_{I ; \mathbf{c}}^{(g, \epsilon)}$ be as in (15.18) and $e_{1}=(1,0,0, \ldots) \in\left(\mathbb{Z}^{\geq 0}\right)^{\infty}$. If $(g, I) \neq(1,(0))$ or $|\underline{\epsilon}| \geq 2$, then (5.3) and the vanishing statement after (5.3) imply that

$$
A_{I ; \underline{\mathbf{c}}+e_{1}}^{(g, \underline{\epsilon}}=(2 g-2+m+|\mathbf{c}|) A_{I ; \mathbf{c}}^{(g, \underline{\mathbf{c}})} .
$$

If $(g, I)=(1,(0))$, then (5.3) , the vanishing statement after (5.3), and (5.4) imply that

$$
A_{I ; \mathbf{c}+e_{1}}^{(g, \underline{\epsilon})}=(2 g-2+m+|\mathbf{c}|) A_{I ; \mathbf{c}}^{(g, \underline{\epsilon})}+C_{(0) ;(1)}^{(1)}(m+|\mathbf{c}|-1) ! \cdot \begin{cases}(-1), & \text { if }|\underline{\epsilon}|=1 ; \\ (m+|\mathbf{c}|-\|\mathbf{c}\|), & \text { if }|\underline{\epsilon}|=0 .\end{cases}
$$

Combining these observations with (2.6) $)$, we obtain

$$
A_{I, \mathbf{b} ; \mathbf{c}+e_{1}}^{(g)}=-(2 g-2+m+|\mathbf{c}|) A_{I, \mathbf{b} ; \mathbf{c}}^{(g)} .
$$

The claim follows from this identity.

Example 5.6. By (5.5) and (5.15),

$$
C_{() ; \underline{\epsilon}}^{(0)}=\left\{\begin{array}{ll}
1, & \text { if } \underline{\epsilon}=\mathbf{0} ; \\
0, & \text { otherwise }
\end{array} \quad C_{(1) ; \underline{\epsilon}}^{(1)}=\frac{1}{24} \cdot \begin{cases}1, & \text { if } \underline{\epsilon}=\mathbf{0} \\
0, & \text { otherwise }\end{cases}\right.
$$

Combining this with (5.18), we obtain

$$
A_{() ; \mathbf{c}}^{(0, \underline{\mathbf{c}})}=\left\{\begin{array}{ll}
(m-3+|\mathbf{c}|) !, & \text { if } \underline{\epsilon}=\mathbf{0} \in\left(\mathbb{Z}^{\geq 0}\right)^{m} ; \\
0, & \text { if } \underline{\epsilon} \neq \mathbf{0} ;
\end{array} \quad A_{(1) ; \mathbf{c}}^{(1, \underline{\epsilon}}= \begin{cases}\frac{(m-1+|\mathbf{c}|) !}{24}, & \text { if } \underline{\epsilon}=\mathbf{0} \in\left(\mathbb{Z}^{\geq 0}\right)^{m} \\
0, & \text { if } \underline{\epsilon} \neq \mathbf{0} .\end{cases}\right.
$$

Since

$$
\left\langle\left\langle\lambda_{0 ;()} ; \widetilde{\tau}_{\mathbf{b}}\right\rangle\right\rangle=\left\{\begin{array}{ll}
|\mathbf{b}| !, & \text { if }|\mathbf{b}| \geq m-3 ; \\
0, & \text { otherwise; }
\end{array} \quad\left\langle\left\langle\lambda_{1 ;(1)} ; \widetilde{\tau}_{\mathbf{b}}\right\rangle\right\rangle=\frac{1}{24} \cdot \begin{cases}|\mathbf{b}| !, & \text { if }|\mathbf{b}| \geq m-1 \\
0, & \text { otherwise }\end{cases}\right.
$$

the $(g, I)=(0,()),(1,(1))$ cases of (2.6) reduce to

$$
\begin{array}{r}
\sum_{\mathbf{b}^{\prime} \in(\mathbb{Z} \geq 0)^{S(\mathbf{c})}}\left((-1)^{\left|\mathbf{b}^{\prime}\right|}\left(\begin{array}{c}
|\mathbf{b}|+\left|\mathbf{b}^{\prime}\right| \\
m-3+|\mathbf{c}|
\end{array}\right) \prod_{(r, j) \in S(\mathbf{c})}\left(\begin{array}{c}
r \\
b_{r, j}^{\prime}
\end{array}\right)\right)=(-1)^{\|\mathbf{c}\|}(m+|\mathbf{c}|-3) !\left(\begin{array}{c}
|\mathbf{b}| \\
m-3+|\mathbf{c}|-\|\mathbf{c}\|
\end{array}\right), \\
\sum_{\mathbf{b}^{\prime} \in(\mathbb{Z} \geq 0)^{S(\mathbf{c})}}\left((-1)^{\left|\mathbf{b}^{\prime}\right|}\left(\begin{array}{c}
|\mathbf{b}|+\left|\mathbf{b}^{\prime}\right| \\
m-1+|\mathbf{c}|
\end{array}\right) \prod_{(r, j) \in S(\mathbf{c})}\left(\begin{array}{c}
r \\
b_{r, j}^{\prime}
\end{array}\right)\right)=(-1)^{\|\mathbf{c}\|}(m+|\mathbf{c}|-1) !\left(\begin{array}{c}
|\mathbf{b}| \\
m-1+|\mathbf{c}|-\|\mathbf{c}\|
\end{array}\right)
\end{array}
$$

for $m \geq 3$ and $m \geq 1$, respectively. These two identities are immediate consequences of the first two statements in Lemma 5.1 
Example 5.7. By (5.5) and (5.15),

$$
C_{(0) ; \underline{\epsilon}}^{(1)}=\frac{1}{24} \cdot \begin{cases}1, & \text { if }|\underline{\epsilon}|=1 \\ -(|\underline{\epsilon}|-2) !, & \text { if } \underline{\epsilon} \in\{0,1\}^{m},|\epsilon| \geq 2 \\ 0, & \text { otherwise. }\end{cases}
$$

Combining this with (5.18), we obtain

$$
A_{(0) ; \mathbf{c}}^{(1, \underline{\epsilon})}=-\frac{1}{24} \sum_{\mathbf{c}^{\prime} \in(\mathbb{Z} \geq 0) \infty}\left(|\underline{\epsilon}|+\left|\mathbf{c}^{\prime}\right|-2\right) !\left(m+|\mathbf{c}|-\left(|\underline{\epsilon}|+\left|\mathbf{c}^{\prime}\right|\right)\right) ! \prod_{r=1}^{\infty}\left(\begin{array}{c}
c_{r} \\
c_{r}^{\prime}
\end{array}\right) r^{c_{r}^{\prime}} \quad \text { if } \underline{\epsilon} \in\{0,1\}^{m}
$$

with $(-1) ! \equiv-1$ and $(-2) ! \equiv 0$; all other coefficients $A_{I ; \mathbf{c}}^{(g, \epsilon)}$ with $(g, I)=(1,(0))$ provided by (5.18) vanish. Thus,

$$
A_{(0) ; 0}^{(1, \underline{\epsilon})}=\frac{(m-|\underline{\epsilon}|) !}{24} \cdot \begin{cases}1, & \text { if } \underline{\epsilon} \in\{0,1\}^{m},|\underline{\epsilon}|=1 \\ -(|\underline{\epsilon}|-2) !, & \text { if } \underline{\epsilon} \in\{0,1\}^{m},|\epsilon| \geq 2 \\ 0, & \text { otherwise. }\end{cases}
$$

If $r \in \mathbb{Z}^{+}$and $e_{r} \in\left(\mathbb{Z}^{\geq 0}\right)^{\infty}$ denotes the $r$-th standard coordinate vector, then

$$
A_{(0) ; e_{r}}^{(1, \underline{\epsilon})}=\frac{(m-|\underline{\epsilon}|) !}{24} \cdot \begin{cases}r, & \text { if } \underline{\epsilon} \in\{0,1\}^{m},|\underline{\epsilon}|=0 ; \\ (m-r), & \text { if } \underline{\epsilon} \in\{0,1\}^{m},|\underline{\epsilon}|=1 ; \\ -(|\underline{\epsilon}|-2) !((|\underline{\epsilon}|-1)(r-1)+m), & \text { if } \underline{\epsilon} \in\{0,1\}^{m},|\epsilon| \geq 2 ; \\ 0, & \text { otherwise. }\end{cases}
$$

\subsection{Sums of residues of generating series}

For $g, m \in \mathbb{Z}^{\geq 0}$ with $2 g+m \geq 3, I \in\left(\mathbb{Z}^{\geq 0}\right)^{g}, \mathbf{c} \in\left(\mathbb{Z}^{\geq 0}\right)^{\infty}$, and $\underline{\epsilon} \in\left(\mathbb{Z}^{\geq 0}\right)^{m}$, let $\widehat{A}_{I ; \mathbf{c}}^{(g, \underline{\mathbf{c}})} \in \mathbb{Q}$ be as in (2.19).

Proposition 5.8. Let $g, m \in \mathbb{Z}^{\geq 0}$ with $2 g+m \geq 3, I \in\left(\mathbb{Z}^{\geq 0}\right)^{g}$, and $\mathbf{b} \in\left(\mathbb{Z}^{\geq 0}\right)^{m}$. If

$$
\zeta, \Psi_{0}, \Psi_{1}, \ldots \in q \mathbb{Q}_{\alpha}(\hbar)[[q]] \quad \text { and } 1+\mathcal{Z}^{*}(\hbar, q)=e^{\zeta(q) / \hbar}\left(1+\sum_{b=0}^{\infty} \Psi_{b}(q) \hbar^{b}\right),
$$

then

$$
\begin{aligned}
& \sum_{m^{\prime}=0}^{\infty} \sum_{\substack{\mathbf{b}^{\prime} \in\left(\mathbb{Z}^{\geq} \geq 0\right)^{m^{\prime}} \\
|\mathbf{b}|+\left|\mathbf{b}^{\prime}\right|=\mu_{g}(I)+m+m^{\prime}}}\left(\frac{\left\langle\left\langle\lambda_{g ; I} ; \widetilde{\tau}_{\mathbf{b}^{\prime}}\right\rangle\right\rangle}{m^{\prime} !} \prod_{k=1}^{k=m^{\prime}} \mathfrak{R}\left\{\frac{(-\hbar)^{-b_{k}^{\prime}}}{b_{k}^{\prime} !} \mathcal{Z}^{*}(\hbar, q)\right\}\right) \\
& =\sum_{\mathbf{c} \in(\mathbb{Z} \geq 0} \sum_{\substack{\boldsymbol{\epsilon} \in(\mathbb{Z} \geq 0)^{m} \\
|\in| \leq \mu_{g}(I)+m \\
\epsilon_{k} \leq b_{k} \\
\forall k \in[m]}}\left(\frac{(-1)^{|\mathbf{c}|+\|\mathbf{c}\|} \widehat{A}_{I ; \mathbf{c}}^{(g, \boldsymbol{\epsilon})}}{\left(1+\Psi_{0}(q)\right)^{2 g-2+m}} \prod_{r=1}^{\infty} \frac{1}{c_{r} !}\left(\frac{\Psi_{r}(q)}{(r+1) !\left(1+\Psi_{0}(q)\right)}\right)^{c_{r}}\right. \\
& \left.\times \zeta(q)^{|\mathbf{b}|-\left(\mu_{g}(I)+m-\|\mathbf{c}\|\right)}\left(\begin{array}{c}
|\mathbf{b}|-|\underline{\epsilon}| \\
\mu_{g}(I)+m-\|\mathbf{c}\|-|\underline{\epsilon}|
\end{array}\right) \prod_{k=1}^{m} \frac{b_{k} !}{\left(b_{k}-\epsilon_{k}\right) !}\right)
\end{aligned}
$$

in $\mathbb{Q}_{\alpha}[[q]]$. 
Proof. Fix $c_{0} \in \mathbb{Z}^{\geq 0}$ and $\mathbf{c} \equiv\left(c_{r}\right)_{r \in \mathbb{Z}^{+}} \in\left(\mathbb{Z}^{\geq 0}\right)^{\infty}$. Let

$$
\begin{gathered}
\Psi^{\mathbf{c}}=\prod_{r=1}^{\infty} \Psi_{r}^{c_{r}}, \quad \omega(\mathbf{c})=\prod_{r=1}^{\infty}((r+1) !)^{c_{r}}, \\
S\left(c_{0}, \mathbf{c}\right)=\left\{(r, j) \in \mathbb{Z}^{\geq 0} \times \mathbb{Z}^{+}:(r, j) \in\{r\} \times\left[c_{r}\right] \forall r \in \mathbb{Z}^{\geq 0}\right\}, \\
A_{\mathbf{b}, I ; c_{0}, \mathbf{c}}^{(g)}=\sum_{\mathbf{b}^{\prime} \in\left(\mathbb{Z}^{\geq 0}\right)^{S\left(c_{0}, \mathbf{c}\right)}}(-1)^{\left|\mathbf{b}^{\prime}\right|} \frac{\left\langle\left\langle\lambda_{g ; I} ; \widetilde{\tau}_{\mathbf{b} \mathbf{b}^{\prime}}\right\rangle\right\rangle}{\left(|\mathbf{b}|+\left|\mathbf{b}^{\prime}\right|-\mu_{g}(I)-m-c_{0}-|\mathbf{c}|\right) !} \prod_{(r, j) \in S\left(c_{0}, \mathbf{c}\right)}\left(\begin{array}{c}
r+1 \\
b_{r, j}^{\prime}
\end{array}\right) .
\end{gathered}
$$

In particular, $\left|S\left(c_{0}, \mathbf{c}\right)\right|=c_{0}+|\mathbf{c}|$ and the numerator above vanishes whenever the argument of the factorial in the denominator is negative. By Corollary 5.5 and Proposition 2.1,

$$
\begin{aligned}
& A_{\mathbf{b}, I ; c_{0}, \mathbf{c}}^{(g)}=(-1)^{c_{0}+|\mathbf{c}|}+\|\mathbf{c}\| \frac{\left(2 g+m-3+c_{0}+|\mathbf{c}|\right) !}{(2 g+m-3+|\mathbf{c}|) !} \\
& \times \sum_{\substack{\epsilon \in(\mathbb{Z} \geq 0) m \\
|\underline{\epsilon}| \leq \mu_{g}(I)+m \\
\epsilon_{k} \leq b_{k} \forall k \in[m]}} \widehat{A}_{I ; \mathbf{c}}^{(g, \hat{\epsilon})}\left(\begin{array}{c}
|\mathbf{b}|-|\underline{\epsilon}| \\
\mu_{g}(I)+m-\|\mathbf{c}\|-|\underline{\epsilon}|
\end{array}\right) \prod_{k=1}^{m} \frac{b_{k} !}{\left(b_{k}-\epsilon_{k}\right) !},
\end{aligned}
$$

with $\widehat{A}_{I ; \mathbf{c}}^{(g, \underline{\epsilon})}$ as in (2.19).

We establish (5.21) by comparing the coefficients of $\Psi_{0}^{c_{0}} \Psi^{\mathbf{c}}$ on the two sides. By (5.20),

$$
\underset{\hbar=0}{\mathfrak{R}}\left\{\hbar^{-b} \mathcal{Z}^{*}(\hbar, q)\right\}=\sum_{r=\max (b-1,0)}^{\infty} \frac{\zeta(q)^{r+1-b}}{(r+1-b) !} \Psi_{r}(q)+ \begin{cases}\zeta(q), & \text { if } b=0 \\ 0, & \text { if } b \geq 1\end{cases}
$$

The coefficient $\operatorname{LHS}_{m^{\prime}}\left(c_{0}, \mathbf{c}\right)$ of $\Psi_{0}^{c_{0}} \Psi^{\mathbf{c}}$ in the $m^{\prime}$-th summand on the left-hand side of (5.21) is a sum over the collections of disjoint subsets $S_{0}, S_{1}, \ldots$ of $\left[\mathrm{m}^{\prime}\right]$ of cardinalities $c_{0}, c_{1}, \ldots$ and tuples $\mathbf{b}^{\prime} \in\left(\mathbb{Z}^{\geq 0}\right)^{S\left(c_{0}, \mathbf{c}\right)}$ such that

$$
|\mathbf{b}|+\left|\mathbf{b}^{\prime}\right|=\mu_{g}(I)+m+m^{\prime} \in \mathbb{Z}^{\geq 0} .
$$

The factors in the $m^{\prime}$-fold product in (5.21) that contribute $\Psi_{r}$ are indexed by the elements of $S_{r}$; the $j$-th such factor arises from $\underset{\hbar=0}{\Re}\left\{\hbar^{-b_{r, j}^{\prime}} \mathcal{Z}^{*}(\hbar, q)\right\}$ with $r \geq b_{r, j}^{\prime}-1$. This leaves $m^{\prime}-c_{0}-|\mathbf{c}|$ factors that contribute $\zeta(q)$ from $\underset{\hbar=0}{\mathfrak{R}}\left\{\mathcal{Z}^{*}(\hbar, q)\right\}$. The associated summand contributing to $\operatorname{LHS}_{m^{\prime}}\left(c_{0}, \mathbf{c}\right)$ is then

$$
\begin{aligned}
\frac{\left\langle\left\langle\lambda_{g ; I} ; \widetilde{\tau}_{\mathbf{b b}^{\prime}}\right\rangle\right\rangle}{m^{\prime} !} \zeta^{m^{\prime}-c_{0}-|\mathbf{c}|} \prod_{(r, j) \in S\left(c_{0}, \mathbf{c}\right)}\left(\frac{(-1)^{b_{r, j}^{\prime}}}{b_{r, j}^{\prime} !} \cdot \frac{\zeta^{r+1-b_{r, j}^{\prime}}}{\left(r+1-b_{r, j}^{\prime}\right) !}\right) \\
=\frac{\zeta^{|\mathbf{b}|-\mu_{g}(I)-m+\|\mathbf{c}\|}}{\omega(\mathbf{c})}(-1)^{\left|\mathbf{b}^{\prime}\right|} \frac{\left\langle\left\langle\lambda_{g ; I} ; \widetilde{\tau}_{\mathbf{b} \mathbf{b}^{\prime}}^{\prime}\right\rangle\right.}{m^{\prime} !} \prod_{(r, j) \in S\left(c_{0}, \mathbf{c}\right)}\left(\begin{array}{c}
r+1 \\
b_{r, j}^{\prime}
\end{array}\right) ;
\end{aligned}
$$

the first expression above is defined to be 0 if $b_{r, j}^{\prime}>r+1$ for some $(r, j) \in S\left(c_{0}, \mathbf{c}\right)$. Since the number of collections of subsets above is

$$
\left(\begin{array}{c}
m^{\prime} \\
c_{0}, \mathbf{c}, m^{\prime}-c_{0}-|\mathbf{c}|
\end{array}\right) \equiv \frac{m^{\prime} !}{c_{0} ! \mathbf{c} !\left(|\mathbf{b}|+\left|\mathbf{b}^{\prime}\right|-\mu_{g}(I)-m-c_{0}-|\mathbf{c}|\right) !},
$$


it follows that the coefficient of $\Psi_{0}^{c_{0}} \Psi^{\mathbf{c}}$ on the left-hand side of (5.21) is

$$
\sum_{m^{\prime}=0}^{\infty} \operatorname{LHS}_{m^{\prime}}\left(c_{0}, \mathbf{c}\right)=\frac{\zeta^{|\mathbf{b}|-\mu_{g}(I)-m+\|\mathbf{c}\|}}{\omega(\mathbf{c}) c_{0} ! \mathbf{c} !} A_{\mathbf{b}, I ; c_{0}, \mathbf{c}}^{(g)} .
$$

The claim now follows from (5.22) and the last statement in Lemma 5.1

Department of Mathematics, Stony Brook University, Stony Brook, NY 11794

azinger@math.stonybrook.edu

\section{References}

[1] M. Atiyah and R. Bott, The moment map and equivariant cohomology, Topology 23 (1984), $1-28$

[2] K. Behrend and B. Fantechi, The intrinsic normal cone, Invent. Math. 128 (1997), no. 1, 45-88

[3] A. Bertram and H. Kley, New recursions for genus-zero Gromov-Witten invariants, Topology 44 (2005), no. 1, 1-24

[4] P. Candelas, X. de la Ossa, P. Green, and L. Parkes, A pair of Calabi-Yau manifolds as an exactly soluble superconformal theory, Nuclear Phys. B359 (1991), 21-74

[5] L. Cherveny, Genus-zero mirror principle with two marked points, math/1001.0242

[6] Y. Cooper and A. Zinger, Mirror symmetry for stable quotients invariants, Michigan Math. J. 63 (2014), no. 3, 571-621

[7] P. Di Francesco and C. Itzykson, Quantum intersection rings, RCP 25, Vol. 46 (1994), Prepubl. Inst. Rech. Math. Av., 153-226

[8] A. Givental, The mirror formula for quintic threefolds, AMS Transl. Ser. 2, 196 (1999), 49-62

[9] A. Givental, Semisimple Frobenius structures at higher genus, IMRN 2001, no. 23, 1265-1286

[10] T. Graber and R. Pandharipande, Localization of virtual classes, Invent. Math. 135 (1999), $487-518$

[11] K. Hori, S. Katz, A. Klemm, R. Pandharipande, R. Thomas, C. Vafa, R. Vakil, and E. Zaslow, Mirror Symmetry, Clay Math. Inst., AMS, 2003

[12] J. Li and G. Tian, Virtual moduli cycles and Gromov-Witten invariants of algebraic varieties, JAMS 11 (1998), no. 1, 119-174

[13] B. Lian, K. Liu, and S.T. Yau, Mirror Principle I, Asian J. of Math. 1, no. 4 (1997), 729-763

[14] D. Maulik and R. Pandharipande, A topological view of Gromov-Witten theory, Topology 45 (2006), no. 5, 887-918

[15] D. Maulik and R. Pandharipande, in progress 
[16] A. Popa, The genus one Gromov-Witten invariants of Calabi-Yau complete intersections, Trans. AMS 365 (2013), no. 3, 1149-1181

[17] A. Popa, Two-point Gromov-Witten formulas for symplectic toric manifolds, math/1206.2703

[18] A. Popa and A. Zinger, Mirror formulas for closed, open, and unoriented Gromov-Witten invariants, Adv. Math. 259 (2014), 448-510

[19] D. Zagier and A. Zinger, Some properties of hypergeometric series associated with mirror symmetry, Modular Forms and String Duality, Fields Inst. Commun. 54 (2008), 163-177

[20] A. Zinger, The reduced genus-one Gromov-Witten invariants of Calabi-Yau hypersurfaces, JAMS 22 (2009), no. 3, 691-737

[21] A. Zinger, Genus-zero two-point hyperplane integrals in the Gromov-Witten theory, Comm. Ann. Geom. 17 (2010), no. 5, 1-45

[22] A. Zinger, The genus 0 Gromov-Witten invariants of projective complete intersections, Geom. Top. 18 (2014), no. 2, 1035-1114 